NATIONAL LABORATORY

\title{
Effect of Weight and Roadway Grade on the Fuel Economy of Class-8 Freight Trucks
}

\section{October 2011}

Prepared by

Oscar Franzese, PhD

Senior Researcher

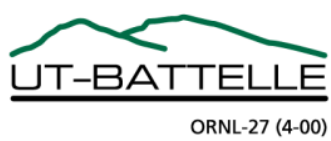




\section{DOCUMENT AVAILABILITY}

Reports produced after January 1, 1996, are generally available free via the U.S. Department of Energy (DOE) Information Bridge.

Web site http://www.osti.gov/bridge

Reports produced before January 1, 1996, may be purchased by members of the public from the following source.

National Technical Information Service

5285 Port Royal Road

Springfield, VA 22161

Telephone 703-605-6000 (1-800-553-6847)

TDD 703-487-4639

Fax 703-605-6900

E-mail info@ntis.gov

Web site http://www.ntis.gov/support/ordernowabout.htm

Reports are available to DOE employees, DOE contractors, Energy Technology Data Exchange (ETDE) representatives, and International Nuclear Information System (INIS) representatives from the following source.

Office of Scientific and Technical Information

P.O. Box 62

Oak Ridge, TN 37831

Telephone 865-576-8401

Fax 865-576-5728

E-mail reports@osti.gov

Web site http://www.osti.gov/contact.html

This report was prepared as an account of work sponsored by an agency of the United States Government. Neither the United States Government nor any agency thereof, nor any of their employees, makes any warranty, express or implied, or assumes any legal liability or responsibility for the accuracy, completeness, or usefulness of any information, apparatus, product, or process disclosed, or represents that its use would not infringe privately owned rights. Reference herein to any specific commercial product, process, or service by trade name, trademark, manufacturer, or otherwise, does not necessarily constitute or imply its endorsement, recommendation, or favoring by the United States Government or any agency thereof. The views and opinions of authors expressed herein do not necessarily state or reflect those of the United States Government or any agency thereof. 


\title{
EFFECT OF WEIGHT AND ROADWAY GRADE ON THE FUEL ECONOMY OF CLASS-8 FREIGHT TRUCKS
}

\author{
Oscar Franzese \\ Diane Davidson
}

Date Published: October 2011

\author{
Prepared by \\ OAK RIDGE NATIONAL LABORATORY \\ Oak Ridge, Tennessee 37831-6283 \\ managed by \\ UT-BATTELLE, LLC \\ for the \\ U.S. DEPARTMENT OF ENERGY \\ under contract DE-AC05-00OR22725
}





\section{CONTENTS}

CONTENTS i

LIST OF FIGURES

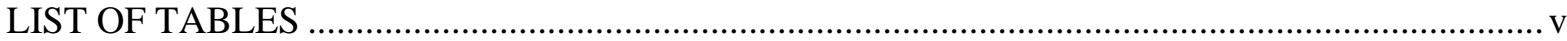

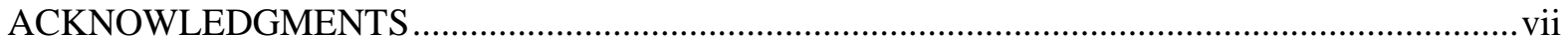

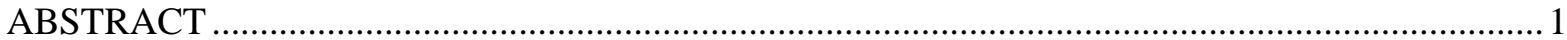

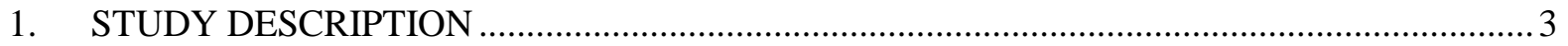

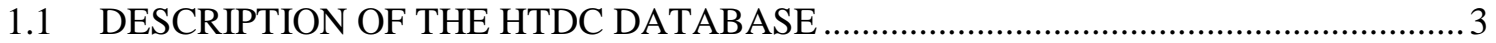

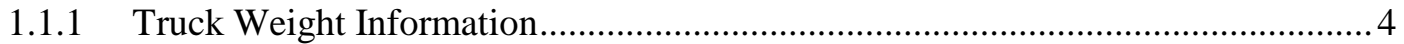

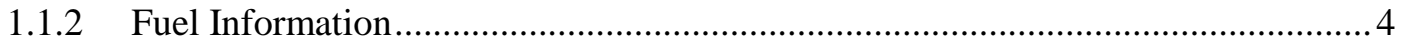

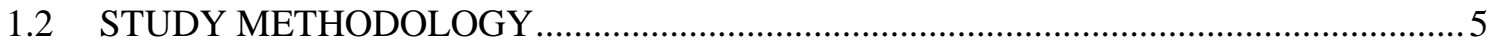

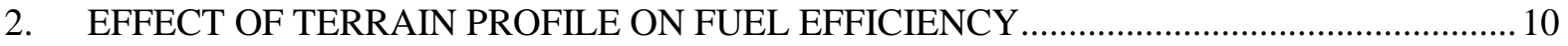

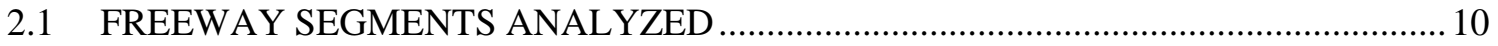

2.1.1 Knoxville, Tennessee to Cleveland, Tennessee Segment...................................... 11

2.1.2 Knoxville, Tennessee to Nashville, Tennessee Segment ...................................... 14

2.1.3 Knoxville, Tennessee to London, Kentucky Segment ......................................... 16

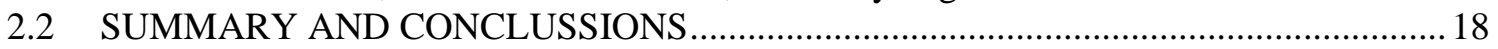

3. EFFECT OF VEHICLE SPEED AND PAYLOAD ON FUEL EFFICIENCY - FLAT

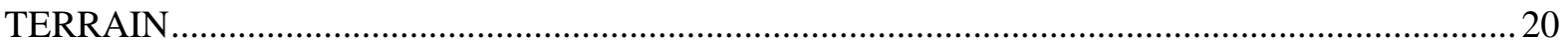

3.1 EFFECT OF VEHICLE SPEED ON FUEL EFFICIENCY - FLAT TERRAIN ...............21

3.1.1 Trips with Tractor and Trailer Weigh Sensors .....................................................25

3.2 EFFECT OF VEHICLE WEIGHT ON FUEL EFFICIENCY - FLAT TERRAIN ............27

3.2.1 FE Extrapolation as a Function of Vehicle Weight ..............................................2. 28

3.3 EFFECT OF VEHICLE SPEED AND PAYLOAD ON FUEL EFFICIENCY - FLAT

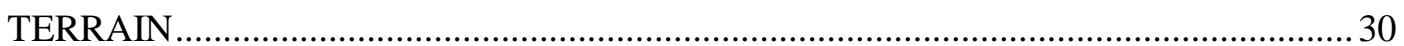

4. EFFECT OF PAYLOAD AND VEHICLE SPEED ON FUEL EFFICIENCY - UPSLOPE

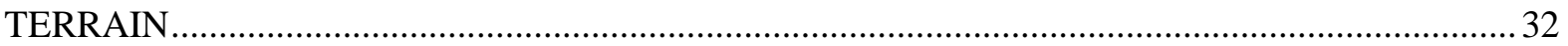

4.1 EFFECT OF VEHICLE SPEED ON FUEL EFFICIENCY - UPSLOPE TERRAIN ........ 32

4.2 EFFECT OF VEHICLE WEIGHT ON FUEL EFFICIENCY - UPSLOPE TERRAIN .... 34

4.3 EFFECT OF VEHICLE SPEED AND VEHICLE WEIGHT ON FUEL EFFICIENCY -

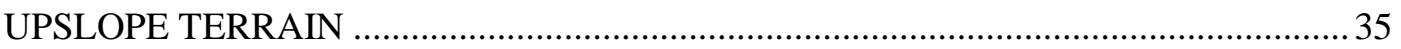

5. COMPARISSON OF FUEL EFFICIENCIES AT DIFFERENT SPEEDS - FLAT TERRAIN 38

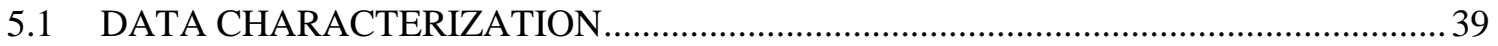

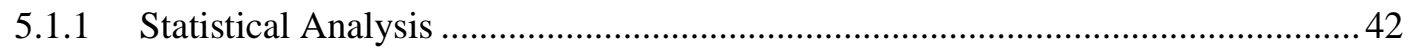

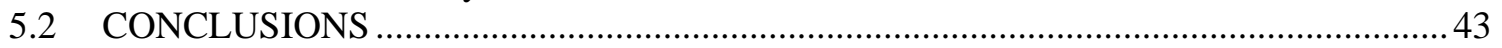

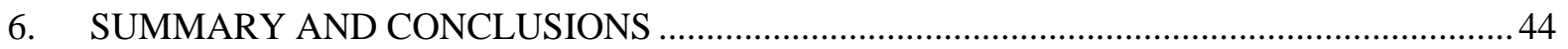

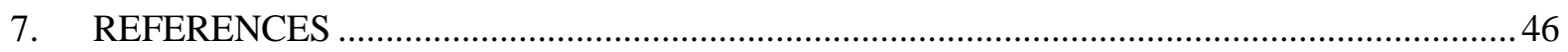

Appendix A. HTDC Database Geographical Coverage and General Statistics ............................. A3

Appendix B. HTDC Data Channels Collected …............................................................................ B3

Appendix C. Effect of Weight and Roadway Grade on the Fuel Economy of Class-8 Freight Trucks

(Interim Report - September 2010) 


\section{LIST OF FIGURES}

Figure

Fig. 1. Vehicle Speed, Instantaneous Fuel Consumption, and Terrain Profile as a Function of

Distance Traveled. (HTDC Truck 4 - 68,620 lb-07/31/2007).

Fig. 2. Vehicle Speed, Instantaneous Fuel Consumption, and Terrain Profile as a Function of

Distance Traveled. Down Slope Terrain Inset (HTDC Truck $4-68,620 \mathrm{lb}-07 / 31 / 2007)$............... 7

Fig. 3. Effect of Terrain-slope Changes on Instantaneous Fuel Consumption. (HTDC Truck $4-$

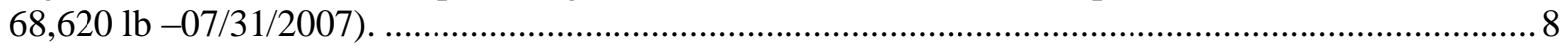

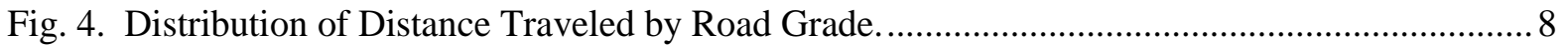

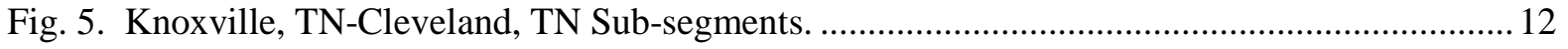

Fig. 6. Knoxville, TN-Cleveland, TN Terrain Profile by Sub-segment. ............................................. 13

Fig. 7. Knoxville, TN-Cleveland, TN Segment 1-2 FE by Trip Speed and Weight........................... 13

Fig. 8. Knoxville, TN-Cleveland, TN Segment 2-3 FE by Trip Speed and Weight........................... 13

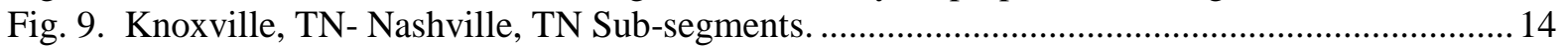

Fig. 10. Knoxville, TN-Nashville, TN Terrain Profile by Sub-segments 1-2 and 2-3..................... 15

Fig. 11. Knoxville, TN-Nashville, TN Segment 1-2 FE by Trip Speed and Weight.......................... 15

Fig. 12. Knoxville, TN-Nashville, TN Segment 2-3 FE by Trip Speed and Weight......................... 15

Fig. 13. Knoxville, TN-Nashville, TN Terrain Profile by Sub-segments 3-4 and 4-5 ..................... 16

Fig. 14. Knoxville, TN-Nashville, TN Segment 3-4 FE by Trip Speed and Weight.......................... 16

(Note: Bubble Size Proportional to FE; Speed Variability: Blue Bubbles $<0.15$, Red Bubbles 0.15 to $0.50) 16$

Fig. 15. Knoxville, TN-Nashville, TN Segment 4-5 FE by Trip Speed and Weight......................... 16 (Note: Bubble Size Proportional to FE; Speed Variability: Blue Bubbles <0.15, Red Bubbles 0.15 to $0.50) 16$

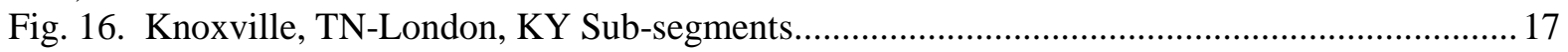

Fig. 17. Knoxville, TN-London, KY Terrain Profile by Sub-segment.............................................. 18

Fig. 18. Knoxville, TN-London, KY Segment 1-2 FE by Trip Speed and Weight.......................... 18

Fig. 19. Knoxville, TN-London, KY Segment 2-3 FE by Trip Speed and Weight............................ 18

Fig. 20. Total Distance Traveled and Total Fuel Consumed vs. Vehicle Speed - Flat Terrain. ......... 21

Fig. 21. Total Distance Traveled and Total Fuel Consumed vs. Vehicle Speed - Flat Terrain, Tractor

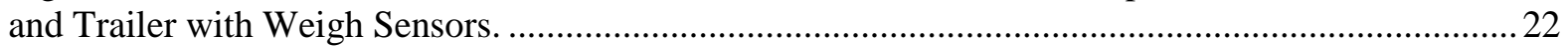

Fig. 22. FE vs. Speed, Vehicle Weight between 20,000 lb to 40,000 lb (Average: 33,700 lb). Flat

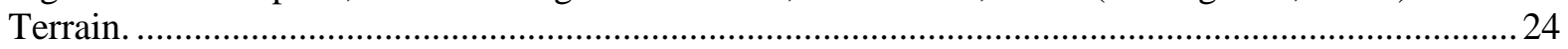

Fig. 23. FE vs. Speed, Vehicle Weight between 40,000 lb to 60,000 lb (Average: 49,700 lb). Flat

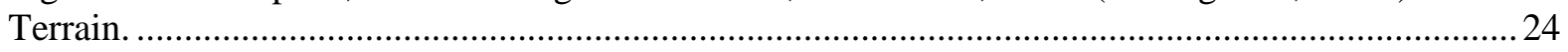

Fig. 24. FE vs. Speed, Vehicle Weight between 60,000 lb to 70,000 lb (Average: 66,400 lb). Flat

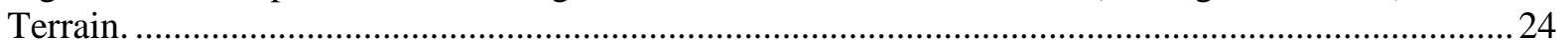

Fig. 25. FE vs. Speed, Vehicle Weight between 70,000 lb to 80,000 lb (Average: 73,600 lb). Flat

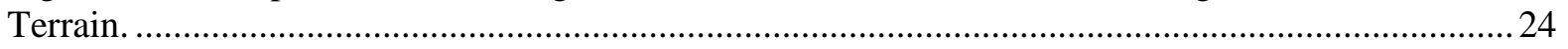

Fig. 26. FE vs. Vehicle Speed for Different Vehicle Weight Levels (with Polynomial Regression

Lines). Flat Terrain............................................................................................................25

Fig. 27. FE vs. Vehicle Speed for Different Vehicle Weight Levels. Trips with Tractor and Trailer

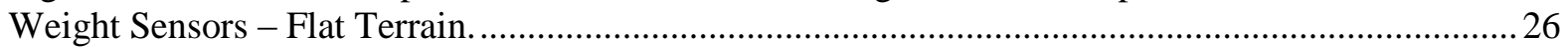

Fig. 28. FE vs. Vehicle Speed for Different Vehicle Weight Levels. All Trips -Flat Terrain........... 26

Fig. 29. FE vs. Vehicle Weight, Vehicle Speed between $55 \mathrm{mph}$ to $60 \mathrm{mph}$ (Average: $58.3 \mathrm{mph}$ ).

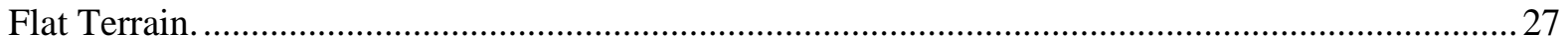

Fig. 30. FE vs. Vehicle Weight, Vehicle Speed between $50 \mathrm{mph}$ to $65 \mathrm{mph}$ (Average: $62.9 \mathrm{mph}$ ). Flat

Terrain. 27 
Fig. 31. FE vs. Vehicle Weight, Vehicle Speed between $65 \mathrm{mph}$ to $70 \mathrm{mph}$ (Average: $68.0 \mathrm{mph}$ ). Flat Terrain.

Fig. 32. FE vs. Vehicle Weight, Vehicle Speed between $70 \mathrm{mph}$ to $75 \mathrm{mph}$ (Average: $72.3 \mathrm{mph}$ ). Flat

Terrain.

Fig. 33. FE vs. Vehicle Weight for Different Speed Intervals (with Polynomial Regression Lines).

Flat Terrain.

Fig. 34. FE vs. Vehicle Weight. Vehicle Speed: $65 \mathrm{mph}$ - Flat Terrain.

Fig. 35. FE vs. Vehicle Speed and Vehicle Weight (Raw Data) - Flat Terrain.

Fig. 36. FE vs. Vehicle Speed and Vehicle Weight (Fitted Data) - Flat Terrain.

Fig. 37. Total Distance Traveled and Total Fuel Consumed vs. Vehicle Speed. Upslope Terrain. ... 33

Fig. 38. FE vs. Vehicle Speed for Different Vehicle Weight Levels (Polynomial Regression Lines).

Upslope Terrain.

Fig. 39. FE vs. Weight for Different Vehicle Speed Intervals (with Polynomial Regression Lines).

Upslope Terrain 35

Fig. 40. FE vs. Vehicle Speed and Vehicle Weight (Raw Data) - Upslope Terrain........................... 36

Fig. 41. FE vs. Vehicle Speed and Vehicle Weight (Fitted Data) - Upslope Terrain.......................... 36

Fig. 42. FE vs. Vehicle Speed for Two Vehicle Weight Levels - Flat Terrain................................... 38

Fig. 43. Dataset Distributions of Vehicle Speed for Four Analysis Speeds. Flat Terrain..................40

Fig. 44. Dataset Distributions of Vehicle Weight for Four Analysis Speeds. Flat Terrain................ 40

Fig. 45. Dataset Distributions of Roadway Grades for Four Analysis Speeds. Flat Terrain............. 41

Fig. 46. Dataset Distributions of Vehicle Fuel Efficiency for Four Analysis Speeds. Flat Terrain...41

Fig. A1. Superimposed Trips (Six Class-8 Trucks, One Year) ........................................................ A3 


\section{LIST OF TABLES}

Table

Page

Table 1. Segment Description and General Statistics.................................................................. 11

Table 2. Knoxville, TN-Cleveland, TN Segment Description and General Statistics......................... 11

Table 3. Knoxville, TN-Nashville, TN Segment Description and General Statistics ........................ 14

Table 4. Knoxville, TN-London, KY Segment Description and General Statistics .......................... 17

Table 5. Fuel Efficiency by Speed for Flat Terrain Vehicle Weight 60,000 lb to 70,000 lb..............23

Table 6. Fuel Efficiency by Speed for Flat Terrain Vehicle Weight 70,000 lb to 80,000 lb.............23

Table 7. Fuel Efficiency by Vehicle Weight Range Speed: $65.0 \mathrm{mph}$ - Flat Terrain ........................29

Table 8. Fuel Efficiency by Speed for Flat Terrain and Vehicle Weight 70,000 lb to 80,000 lb ........ 39

Table 9. Comparison of Fuel Efficiencies for Different Speed Levels Average Vehicle Weight

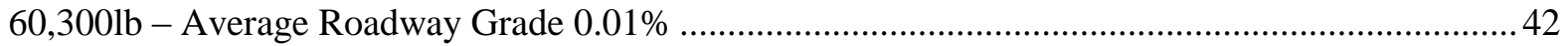

Table A1. General Statistics for the Instrumented Tractors ........................................................ A3

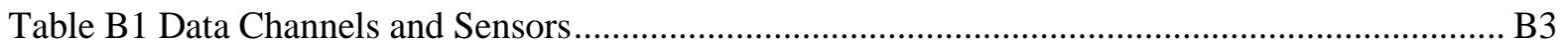

Table B2 Additional Information Added to Each Record Collected.................................................. B4 


\section{ACKNOWLEDGMENTS}

This project was sponsored by the Office of Freight Management and Operations, Federal Highway Administration, U.S. Department of Transportation. The author would like to thank that office for funding and supporting this project, and in particular Mr. Mike Sprung who provided guidance and meaningful feedback. His suggestions and comments helped to greatly improve the research conducted here. The remaining errors, however, are the author's. 



\begin{abstract}
In 2006-08, the Oak Ridge National Laboratory, in collaboration with several industry partners, collected real-world performance and situational data for long-haul operations of Class-8 trucks from a fleet engaged in normal freight operations. Such data and information are useful to support Class- 8 modeling of combination truck performance, technology evaluation efforts for energy efficiency, and to provide a means of accounting for real-world driving performance within combination truck research and analyses.

The present study used the real-world information collected in that project to analyze the effects that vehicle speed and vehicle weight have on the fuel efficiency of Class- 8 trucks. The analysis focused on two type of terrains, flat (roadway grades ranging from $-1 \%$ to $1 \%$ ) and mild uphill terrains (roadway grades ranging from $1 \%$ to $3 \%$ ), which together covered more than $70 \%$ of the miles logged in the 200608 project (note: almost $2 / 3$ of the distance traveled on mild uphill terrains was on terrains with $1 \%$ to $2 \%$ grades). In the flat-terrain case, the results of the study showed that for light and medium loads, fuel efficiency decreases considerably as speed increases. For medium-heavy and heavy loads (total vehicle weight larger than $65,000 \mathrm{lb}$ ), fuel efficiency tends to increase as the vehicle speed increases from 55 $\mathrm{mph}$ up to about 58-60 mph. For speeds higher than $60 \mathrm{mph}$, fuel efficiency decreases at an almost constant rate with increasing speed. At any given speed, fuel efficiency decreases as vehicle weight increases, although the relationship between fuel efficiency and vehicle weight is not linear, especially for vehicle weights above $65,000 \mathrm{lb}$.

The analysis of the information collected while the vehicles were traveling on mild upslope terrains showed that the fuel efficiency of Class- 8 trucks decreases abruptly with vehicle weight ranging from light loads up to medium-heavy loads. After that, increases in the vehicle weight only decrease fuel efficiency slightly. Fuel efficiency also decreases significantly with speed, but only for light and medium loads. For medium-heavy and heavy, FE is almost constant for speeds ranging from 57 to about $66 \mathrm{mph}$. For speeds higher than $66 \mathrm{mph}$, the FE decreases with speed, but at a lower rate than for light and medium loads.
\end{abstract}

Statistical analyses that compared the fuel efficiencies obtained when the vehicles were traveling at 59 mph vs. those achieved when they were traveling at $65 \mathrm{mph}$ or $70 \mathrm{mph}$ indicated that the former were, on average, higher than the latter. This result was statistically significant at the $99.9 \%$ confidence level (note: the Type II error -i.e., the probability of failing to reject the null hypothesis when the alternative hypothesis is true - was $18 \%$ and $6 \%$, respectively). 


\section{STUDY DESCRIPTION}

In 2006-08, the Oak Ridge National Laboratory (ORNL), in partnership with several industry partners and the U.S. Department of Energy (DOE), collected real-world performance and situational data for longhaul operations of Class- 8 trucks from a fleet engaged in normal freight operations. Almost 60 channels of information, including vehicle speed, vehicle weight, spatial location, fuel consumption, and many others were collected at $5 \mathrm{~Hz}$, resulting in a large database (295 GB) of real-world Class-8 truck operation information that covered over 1000 trips which logged close to 700,000 miles, mostly on freeways.

The objective of the present study was to investigate the effects that vehicle weight, roadway grade (terrain profile), and vehicle speed have on the fuel efficiency (FE) of Class- 8 freight trucks. The research was conducted by ORNL using the extensive database of information collected in the DOE Heavy-Truck Duty Cycle (HTDC) project, and it was divided into three sub-studies. In the first one, inhouse developed software that allows identifying data that is spatially tagged was used to extract information from specific trips. In particular, trips that traversed the same segment of freeway and for which the weight of the tractor and trailer was known (through on-board weigh sensors) were extracted from the database and fuel efficiencies and other statistics were computed for each trip. These statistics were then compared across different segments that presented different terrain profiles to investigate the effect of roadway grade and speed on the FE of large trucks.

In the second sub-study, the HTDC data was parsed by roadway grade categories such as, for example, severe and mild upslope, severe and mild downslope, and flat terrain, vehicle speed intervals (of 1 and 2 $\mathrm{mph}$ ), and vehicle weight levels going from tractor only to fully loaded vehicles by 5,000 lb. For two of the terrain categories (i.e., flat terrain and mild upslope terrain) which cover over $70 \%$ of the total miles logged in the HTDC project, the effect of vehicle weight and vehicle speed on FE was investigated using the parsed data.

Some of the findings of the second sub-study showed that for medium-heavy to heavy loads (i.e., total vehicle weight larger than $65,000 \mathrm{lb}$ ), FE increased with speed up to about $59 \mathrm{mph}$ and then it started to decrease for higher speeds (note: engine efficiency and vehicle aerodynamics, among other factors determine optimal operation speeds). Statistical analyses were conducted to determine the level of significance of this effect. The analyses compared FEs obtained when the vehicle was traveling at 59 mph vs. FEs obtained when traveling at highway speed limits (i.e., $55 \mathrm{mph}, 65 \mathrm{mph}$, and $70 \mathrm{mph}$ ).

\subsection{DESCRIPTION OF THE HTDC DATABASE}

Six Class-8 trucks from a selected fleet, which operates within a large area of the country extending from the east coast to the Mountain Time Zone and from Canada to the US-Mexican border (see Appendix A), were instrumented to collect 56 channels of data for over a year at a rate of $5 \mathrm{~Hz}$ (or 5 readings per second) using an ORNL-developed data acquisition system (DAS). Those channels included information such as instantaneous fuel rate, engine speed, gear ratio, vehicle speed, and other information read from the vehicle's databus; weather information (wind speed, precipitation, air temperature ${ }^{1}$, etc.) gathered

\footnotetext{
${ }^{1}$ Although these factors were not considered in the analysis, they can have considerable effect in fuel efficiency. However, in this study the main objective was to analyze the effect of terrain profile and vehicle weight on fuel efficiency and the data was not clustered across factors such as wind speed and temperature. Because of the large size of the database, the results are unbiased when considered these factors, since all terrain types and vehicle weights analyzed had the same "opportunity" to be collected under all the possible environmental conditions.
} 
from an on-board weather station; spatial information (latitude, longitude, altitude) acquired from a GPS (Global Positioning System) device; and instantaneous tractor and trailer weight obtained from devices mounted on the six participating tractors and ten trailers (see Appendix B for more details). In addition to the data collected directly from the on-board sensors, ORNL added other information to the database that was obtained from the carrier and by post-processing the raw data. This included type of roadway (freeways or surface streets), spatial location specificity (urban or rural areas), roadway grade, type of tires, driver ID, and others. Three of the six instrumented tractors and five of the ten instrumented trailers were mounted with new generation single wide-based tires and the others were mounted with new regular dual tires. Over the duration of the project, the six tractors traveled about 690,000 miles collecting over 295GB of uncompressed data (see Appendix A for general statistics regarding the data collected in this project).

\subsubsection{Truck Weight Information}

One of the important variables in Class- 8 truck fuel economy variations is the weight of the payload. In the HTDC project, weight information was collected using a device (the AirWeigh sensor) which was mounted on the six participating tractors, and provided instantaneous weight measurements at the steer and drive axles. This information was saved as two of the 56 DAS data channels. The device was also mounted on the ten instrumented trailers; therefore, when one of these ten trailers was coupled to one of the six tractors, the DAS collected weight data for the entire truck. However, the mating of an instrumented tractor with an instrumented trailer was not a very common event (less than $6 \%$ frequency of occurrence), and as a result, most of the time the six tractors were coupled to non-instrumented trailers (i.e., trailers without the weight-measuring device on board). For those occasions, only weight at the steer and drive axles were registered.

Using the weight information that was collected, a truck total weight prediction model was developed by ORNL that used the tractor weight, which was always available, and principles of inertial physics. From the project database, 272 long-haul trips for which weight information existed for both the tractor and trailer were selected to calibrate the weight model. The weight model was tested against information from another 98 trips that also had complete (i.e., tractor and trailer) weight information showing, on average, an error (i.e., difference between measured and predicted truck weight) of about 5\%. The truckweight model developed was then used to assign a trailer weight to each one of the records in the database of data collected in this project.

\subsubsection{Fuel Information}

For the HTDC project, fuel consumption information (obtained by integrating one of the collected channels: the instantaneous fuel consumption rate, measured in liters/hour) was gathered from the vehicle databus and saved every 0.2 seconds by the DAS. In order to determine the absolute FE of any vehicle, the fuel consumption needs to be measured accurately. Several studies have shown that the errors introduced by measuring fuel consumption with databus information are small. For example, Bohman [1] reports that databus fuel consumption estimation was within $1 \%$ of the readings obtained using a fuel measurement tube (i.e., a two-meter long tube with known diameter that is used instead of the fuel tank). Hogan et al. [2] used databus readings to collect engine speed, throttle position, and fuel rate data. The latter was compared against the actual fuel rate, which was measured by suspending the fuel tank and attaching it to a strain gage meter that was accurate to $0.0023 \mathrm{~kg}$ or $0.005 \mathrm{lb}$. The comparison of measured and CAN fuel rates was shown to be highly correlated ( 0.992 correlation coefficient) thus providing a high level of confidence in the measurements of fuel consumption using the vehicle databus information. 
Similarly, a 2002 EPA study indicates that the fuel consumption obtained from databus information is accurate, as long as severe "spikes" in the data stream are capped [3]. For DOE study, this precaution was followed by restricting the maximum rate of change for fuel flow to approximately 0.018 $(\mathrm{gal} / \mathrm{sec}) / \mathrm{sec}$. Browand et al. [4] indicate that the use of the databus fuel rate signal is accurate and reliable, particularly if differences in fuel consumptions are measured rather than absolute values. In the HTDC study the data was collected using six identical tractors (all 2005 Volvos) and therefore the measurements of fuel consumed using the vehicle databus information was uniform across all the participating vehicles.

\subsection{STUDY METHODOLOGY}

In a preliminary study (see Appendix C), the information contained in the HTDC database was parsed by type of terrain, tractor/trailer tire type, and vehicle speed. This study showed that for all the terrain types analyzed, the vehicle FE as a function of speed presented three distinct regions: an increasing fuelefficiency region ( 0 to just over $55 \mathrm{mph}$ ) which is mostly a vehicle-acceleration region; a flat fuelefficiency region (about 55-60 mph to $70 \mathrm{mph}$ ) in which the FE is almost constant and which is mostly a vehicle-cruising region; and a high fuel-efficiency region (above $70-75 \mathrm{mph}$ ). The latter appeared to contradict common sense since it is expected that FE decreases with speed, especially for speeds higher than $75 \mathrm{mph}$. At the time of this preliminary study it was speculated that these high fuel efficiencies were the result of the vehicles transitioning from a downsloping terrain to the type of terrain for which the analysis was conducted (i.e., flat, upslope, and downslope terrain type) thereby achieving high fuel efficiencies by using the inertia that the vehicle had and not needing to use fuel while entering the new terrain-type region.

A more detailed analysis conducted subsequently not only corroborated this assumption, but also allowed to determine the area of influence, in terms of FE, that one type of terrain has on a different type of terrain immediately downstream. Consider Fig. 1, which shows data collected for a particular trip conducted by HTDC Truck 4. The figure shows the vehicle speed, instantaneous fuel consumption, and terrain profile as a function of the distance traveled by the vehicle from the start of the trip. It is possible to observe that every time there is a downslope terrain the instantaneous fuel consumption (i.e., fuel rate) drops to zero or almost zero. That is, while traveling on downslope terrains, the vehicle moves only by inertial forces transforming potential energy into kinetic energy (i.e., gravity effect) without consuming any fuel.

In order to study the effect that a change in terrain slope has on the vehicle FE, consider the segment of terrain highlighted in Fig. 2 (distance traveled approximately $45 \mathrm{mi}$ to $55 \mathrm{mi}$ ), which is further expanded in Fig. 3. In the latter figure it is possible to appreciate that when the terrain profile changes from upslope terrain to downslope terrain it takes less than $1 / 4$ of a mile for the instantaneous fuel consumption rate to drop to zero. At the other end, a change from a downsloping terrain to an upslope terrain produces an increase in instantaneous fuel consumption (i.e., vehicle accelerating) in about $1 / 8$ of a mile from the point at which the terrain profile changes. (Note: the large majority of the trips in the HTDC database are freeway trips. Therefore these distances discussed here were traversed at highway speeds; in the case of Fig. 1 to Fig. 3 at about 55 to $68 \mathrm{mph}$ ).

Based on these findings, a new methodology, different from the one used in the preliminary study, was applied to the second and third sub-studies discussed in the introduction of this chapter. First, because as depicted in Fig. 1 while traveling on downslope terrains the vehicles consume very little fuel, the analysis concentrated only of flat terrain (i.e., terrain that has a slope between $-1 \%$ and $+1 \%$ ) and mild upslope terrain (i.e., terrain that has a slope between $+1 \%$ and $+3 \%$ ). Analysis of miles traveled by type of terrain shows that over 70\% (i.e., about 490,000 traveled miles in the HTDC database) corresponds to flat terrain (51\% of the total distance traveled in the HTDC database) and mild upslope terrain ( $21 \%$ of the distance 
traveled) as defined previously (see Fig. 4). Second, to eliminate the effect that previously traveled type of terrain has on the FE of currently traveled terrain, the information corresponding to the first $1 / 4$ of a mile of the currently traveled terrain was eliminated and was not used in the computations. Finally, only cases (i.e. speed bins) in which there were more than 10 miles of distance traveled at that speed were included in the analysis. 


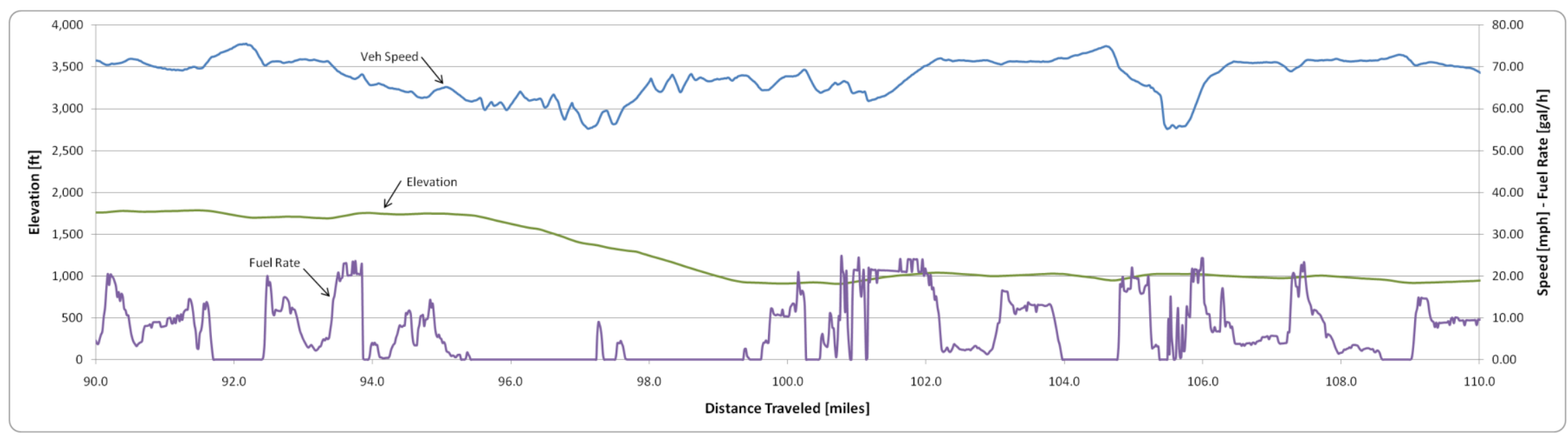

Fig. 1. Vehicle Speed, Instantaneous Fuel Consumption, and Terrain Profile as a Function of Distance Traveled. (HTDC Truck $4-68,620 \mathrm{lb}-07 / 31 / 2007$ ).

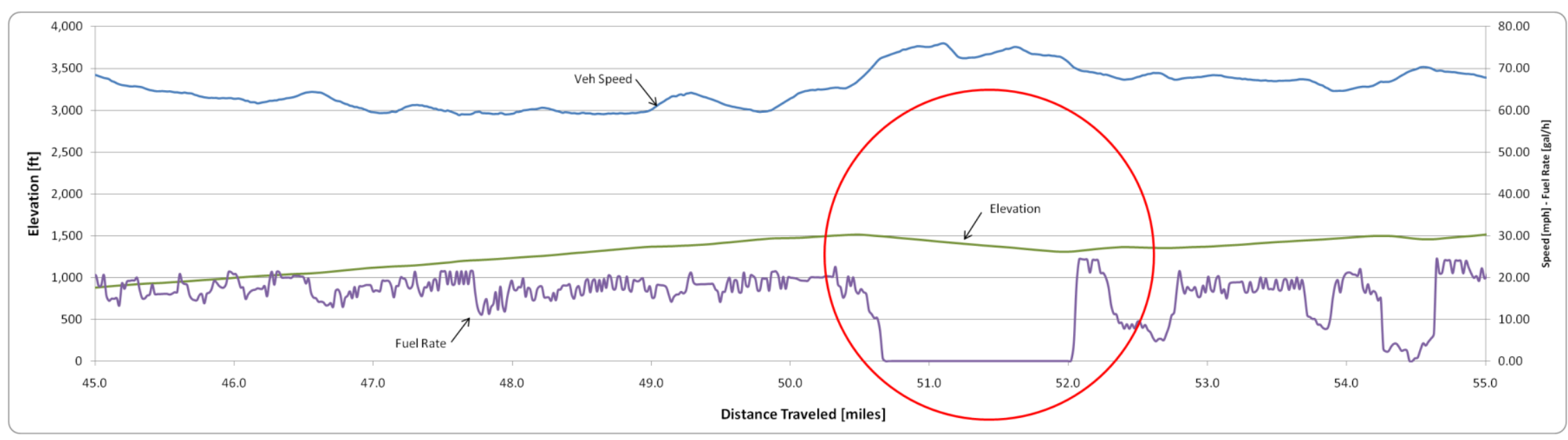

Fig. 2. Vehicle Speed, Instantaneous Fuel Consumption, and Terrain Profile as a Function of Distance Traveled. Down Slope Terrain Inset (HTDC Truck $4-68,620 \mathrm{lb}-07 / 31 / 2007)$. 


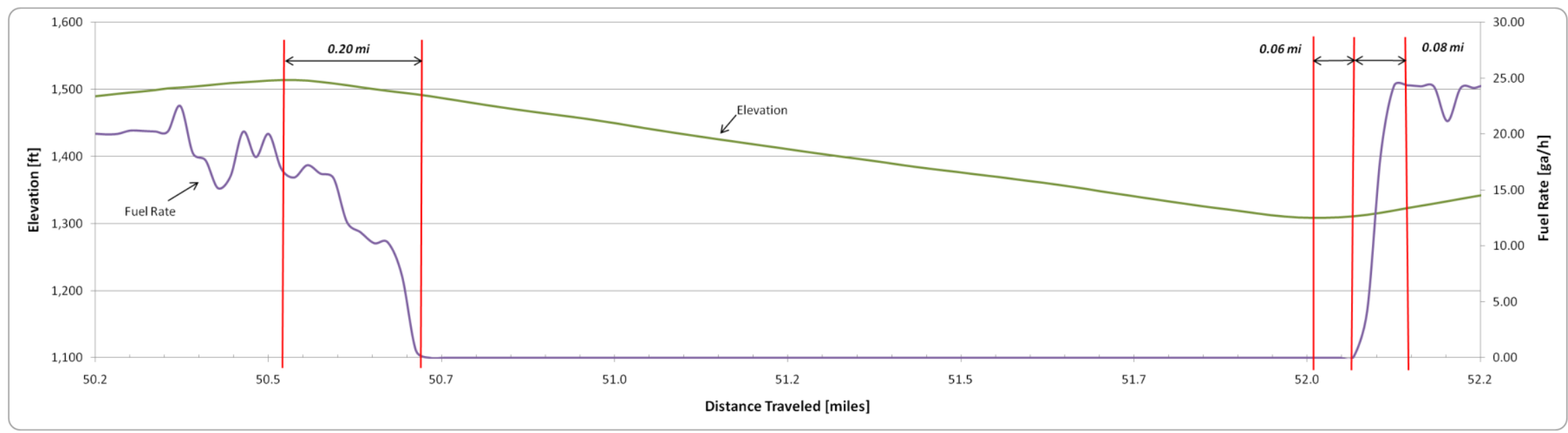

Fig. 3. Effect of Terrain-slope Changes on Instantaneous Fuel Consumption.

(HTDC Truck $4-68,620 \mathrm{lb}-07 / 31 / 2007$ ).

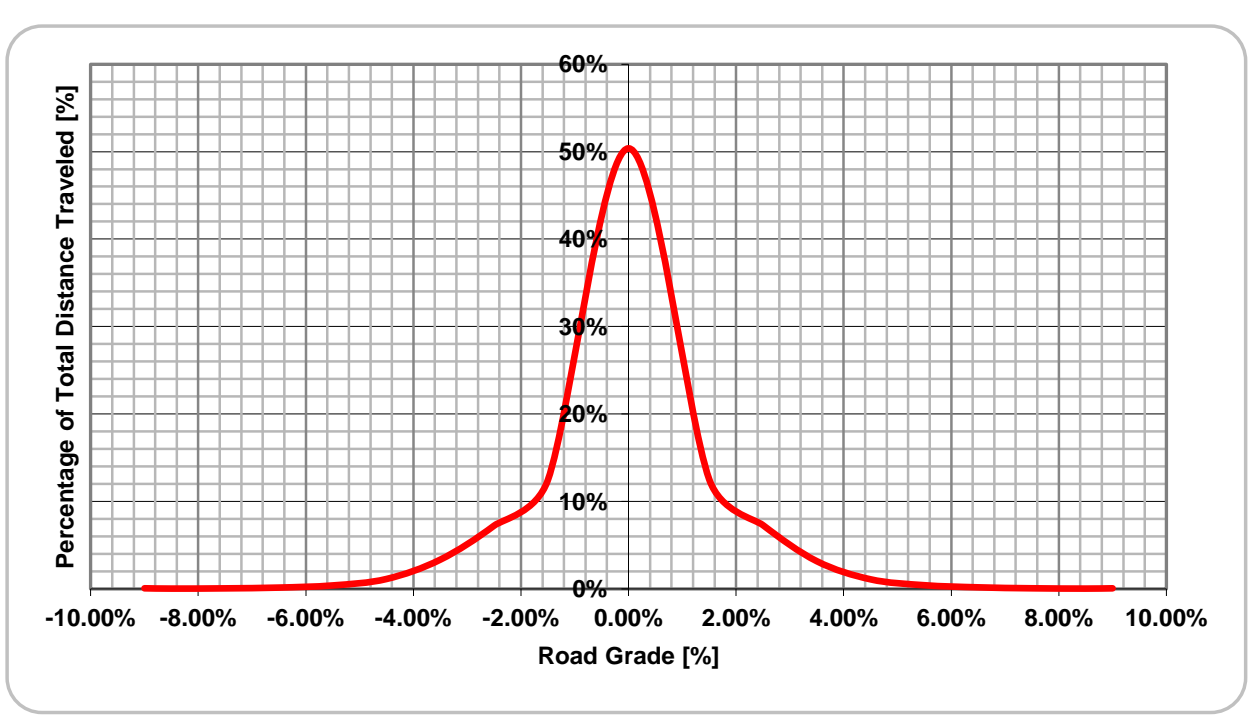

Fig. 4. Distribution of Distance Traveled by Road Grade. 


\section{EFFECT OF TERRAIN PROFILE ON FUEL EFFICIENCY}

The terrain profile for any trip plays a very large role in the achievable FE of any vehicle, and in particular for large trucks. To study this effect in an aggregated form, several segments of freeways were selected from the HTDC database and all the trips in which both the tractor and trailer were instrumented with the AirWeigh sensors were considered. This eliminated any assumptions regarding the vehicle weight since direct measurements were made using these sensors. Also, and by considering long segments of roadway, the issues related to the effect of terrain profile changes on FE were also avoided. The tradeoff, however, is that with this approach it is not possible to study in detail the effect that certain type of terrains (e.g., flat terrain, upslope terrain) have on FE. In order to mitigate this shortcoming, three segments were selected that presented distinct type of terrains. The characteristics of these segments are described in the next section.

The three segments were divided into sub-segments with a length of 40 miles and for each trip that traversed these sub-segments, the FE (in mpg) was computed as the total fuel consumed (in gallons) while a vehicle was traveling on that sub-segment divided by 40 miles (the length of the sub-segment). The vehicle weight was measured by reading the tractor and trailer AirWeigh sensors that provided steer, drive, and trailer axle loads. For each vehicle traveling on a particular sub-segment the average speed was also computed, together with its standard deviation. This allowed computing the speed variability for that vehicle and sub-segment as the ratio of the speed standard deviation and the average speed. High speed variability (i.e., $>0.50$ ) was an indication that the vehicle encountered unusual congestion while traveling on that particular sub-segment. Because congestion has a high impact on FE, those trips with high speed variability were not considered.

The results of the analysis are presented in charts that show for each sub-segment the vehicle weight and vehicle average speed. At the intersection of these two values the charts present the achieved FE for that particular vehicle which is displayed as a bubble with its size being proportional to that FE. All the graphs included in this chapter are drawn to the same scale, so the size of the FE "bubbles" can be compared among all the different sub-segments. In those graphs, trips with speed variability of more than 0.50 are not shown. Trips in which the speed variability was between 0.15 and 0.50 were included, but the corresponding calculated FE "bubble" is displayed in red as opposed to blue for trips with speed variability of less than 0.15 .

\subsection{FREEWAY SEGMENTS ANALYZED}

Three distinct segments were considered for the analysis, all of which started in Knoxville, Tennessee. One of the three contained four forty-mile sub-segments (trips to Nashville, TN on I-40 westbound), while the other two were divided into two forty-mile sub-segments (trips to Cleveland, Tennessee on I-75 southbound and trips to London, Kentucky on I-75 northbound). The characteristics of these three segments are summarized in Table 1. For each segment, the table shows the total length, the number of trips extracted from the HTDC database (all the trips started upstream of the starting point of the origin of the segment and ended downstream of the destination point of each segment), the overall average speed for the segment, and the overall average vehicle weight. 
Table 1. Segment Description and General Statistics

\begin{tabular}{llrrrrrrr}
\hline \multicolumn{1}{c}{ Origin } & Destination & Interstate & $\begin{array}{c}\text { Number } \\
\text { of Trips }\end{array}$ & $\begin{array}{c}\text { Total } \\
\text { Length } \\
\text { [mile] }\end{array}$ & $\begin{array}{c}\text { Average } \\
\text { Speed } \\
\text { [mph] }\end{array}$ & $\begin{array}{c}\text { Average } \\
\text { Vehicle } \\
\text { Weight [lb] }\end{array}$ & $\begin{array}{c}\text { Average } \\
\text { [mpg] }\end{array}$ & Type of Terrain \\
\hline $\begin{array}{l}\text { Knoxville, } \\
\text { TN }\end{array}$ & $\begin{array}{l}\text { Cleveland, } \\
\text { TN }\end{array}$ & I-75 SB & 62 & 80 & 60.1 & 63,241 & 7.7 & Flat \\
$\begin{array}{l}\text { Knoxville, } \\
\text { TN }\end{array}$ & $\begin{array}{l}\text { Nashville, } \\
\text { TN }\end{array}$ & I-40 WB & 28 & 160 & 67.3 & 68,954 & 6.8 & $\begin{array}{c}\text { Flat-Upslope- } \\
\text { Downslope }\end{array}$ \\
$\begin{array}{l}\text { Knoxville, } \\
\text { TN }\end{array}$ & $\begin{array}{l}\text { London, } \\
\text { KY }\end{array}$ & I-75 NB & 50 & 80 & 61.5 & 56,969 & 6.9 & $\begin{array}{c}\text { Rolling-Upslope- } \\
\text { Downslope }\end{array}$ \\
\hline
\end{tabular}

Table 1 also includes a general description of the type of terrain for each segment. For the Knoxville, $\mathrm{TN}-\mathrm{Cleveland}$, TN segment the terrain was classified as "flat" since there is only a drop in elevation of about $200 \mathrm{ft}$ in 80 miles (see Fig. 6). The Knoxville, TN-Nashville, TN segment includes a geological formation known as the Cumberland Plateau which has an upslope segment of about 7 miles in which the elevation increases by $800 \mathrm{ft}$ when traveling westbound on I-40 (an average grade of $+2.2 \%$, see Fig. 10). At the end of the plateau, there is a downhill segment of approximately the same characteristics (Fig. 11). The Knoxville, TN-London, KY segment crosses the Smoky Mountains and contains an upslope segment in which the elevation changes from $900 \mathrm{ft}$ to $1,700 \mathrm{ft}$ in 5 miles when traveling northbound on I-75 (an average grade of $3 \%$. A similar downslope segment exists after crossing the crest of the mountains (see Fig. 17).

\subsubsection{Knoxville, Tennessee to Cleveland, Tennessee Segment}

The Knoxville, TN-Cleveland, TN segment had two sub-segments of 40 miles each. The HTDC database contained 62 trips which originated upstream of Knoxville, TN and ended downstream of Cleveland, TN (i.e., trips that completely traveled the two sub-segments considered). Table 2 shows that for subsegment 1-2 (see Fig. 5 for geographic details) the average speed when considered the 62 trips was 64.6 mph with an average fuel consumption of $7.9 \mathrm{mpg}$ for an average vehicle weight of 63,200 lb. In this segment only two trips presented speed variability between 0.15 and 0.50 . In contrast, for sub-segment 2 3 , four trips had speed variability ranging from 0.15 to 0.50 and 23 had speed variability larger than 0.50 . That is, more than 20 trips out of the 62 considered encountered heavy congestion when traveling on segment 2-3 (the average speed was $55.6 \mathrm{mph}$, almost $10 \mathrm{mph}$ less than for segment 1-2 which has the same topographical characteristics and the same maximum speed of $70 \mathrm{mph}$ ). This was due to roadway construction and maintenance during some part of the data collection period. None of the 23 trips for which the speed variability was larger than 0.50 were included in the graphs. For the computation of the overall average speed, vehicle weight, and FE of the sub-segment, those 23 trips were considered (second row in Table 2) and omitted (third row in the table). The effect of congestion was to reduce the average FE by $0.4 \mathrm{mpg}$ (from $7.9 \mathrm{mpg}$ in sub-segment 1-2 to $7.5 \mathrm{mpg}$ in sub-segment 2-3).

Table 2. Knoxville, TN-Cleveland, TN Segment Description and General Statistics

\begin{tabular}{crrrrrrrr}
\hline Segment & $\begin{array}{c}\text { Segment } \\
\text { Length } \\
\text { [mile] }\end{array}$ & \multicolumn{1}{c}{$\begin{array}{c}\text { Number of } \\
\text { Trips }\end{array}$} & $\begin{array}{c}\text { Average } \\
\text { Speed [mph] }\end{array}$ & $\begin{array}{c}\text { Average Vehicle } \\
\text { Weight [lb] }\end{array}$ & $\begin{array}{c}\text { Average FE } \\
\text { [mpg] }\end{array}$ & \multicolumn{3}{c}{ Speed Variability [\# of Trips] } \\
\hline $\mathbf{1 - 2}$ & 40.0 & 62 & 64.6 & 63,241 & 7.9 & 60 & 2 & 0 \\
\hline $\mathbf{2 - 3}$ & 40.0 & 62 & 55.6 & 63,241 & 7.5 & 35 & 4 & 23 \\
\hline $\mathbf{2 - 3}$ NOL $^{*}$ & 40.0 & 39 & 68.9 & 66,279 & 7.9 & 35 & 4 & 23 \\
\hline
\end{tabular}

*NOL: No Outliers (i.e., trips with Speed Variability > 0.5). 


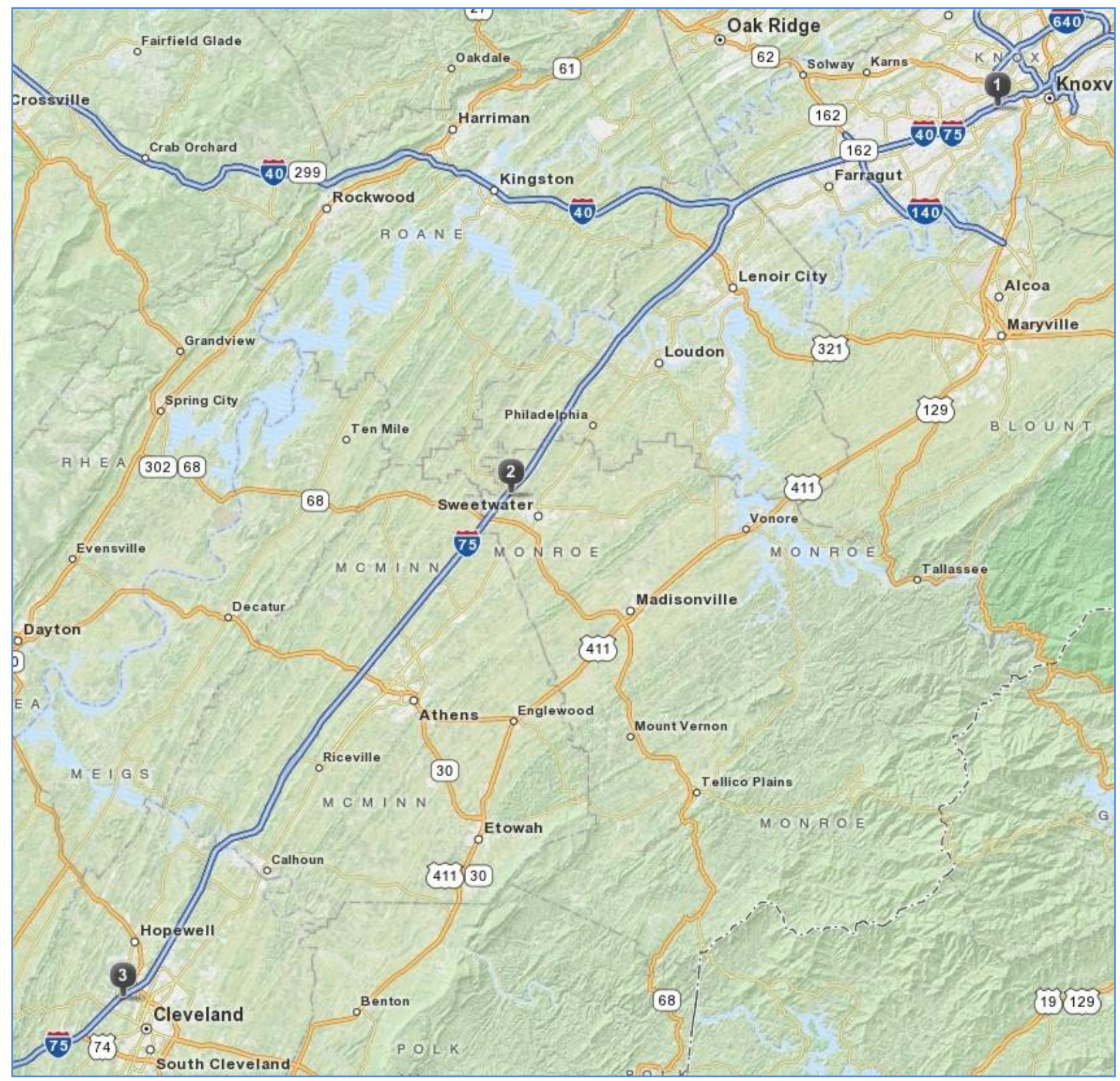

Fig. 5. Knoxville, TN-Cleveland, TN Sub-segments.

Fig. 6 depicts the terrain profile for the Knoxville, TN-Cleveland, TN segment, with an exaggerated scale for altitude (ordinate in feet) compared to the distance traveled (abscissa in miles). As discussed previously, the terrain corresponds to what was defined in the first section of this report as a "flat" terrain. The figure also shows the start and ending point of the two sub-segments: 1-2 and 2-3.

Fig. 7 and Fig. 8 present the results of the analysis for sub-segments 1-2 and 2-3, respectively. Consider Fig. 7 and in that figure consider, for example, the trip in which the vehicle weight was 40,000 lb and the average speed for that vehicle was approximately $66 \mathrm{mph}$. The average FE for that particular vehicle while traveling on sub-segment 1-2 was 8.7 mpg. Larger "bubbles" indicate better FEs while smaller ones indicate worse FEs. In general, and as expected, FE decreases with total vehicle weight (smaller 
"bubbles" at the top of the chart). Regarding speed, FE increases as speed increases and then it starts to decrease again. This speed effect on FE is smaller than the weight effect; it is further discussed in the next sections of this report.

Notice also that, as discussed before, trips with speed variability ranging between 0.15 and 0.50 are shown in the figures as red "bubbles" (e.g., for sub-segment 1-2 there were two such trips). There were four such cases for sub-segment 2-3 (see Table 2 and Fig. 8). However, for this sub-segment there were 23 cases in which the speed variability was above 0.50; those trips are not included in Fig. 8. In Fig. 8, the 40,000 lb trip identified earlier has an average speed of about $74 \mathrm{mph}$ and the FE is $8.2 \mathrm{mpg}$ (a decrease of approximately $5 \%$ when compared with the FE obtained in sub-segment 1-2)

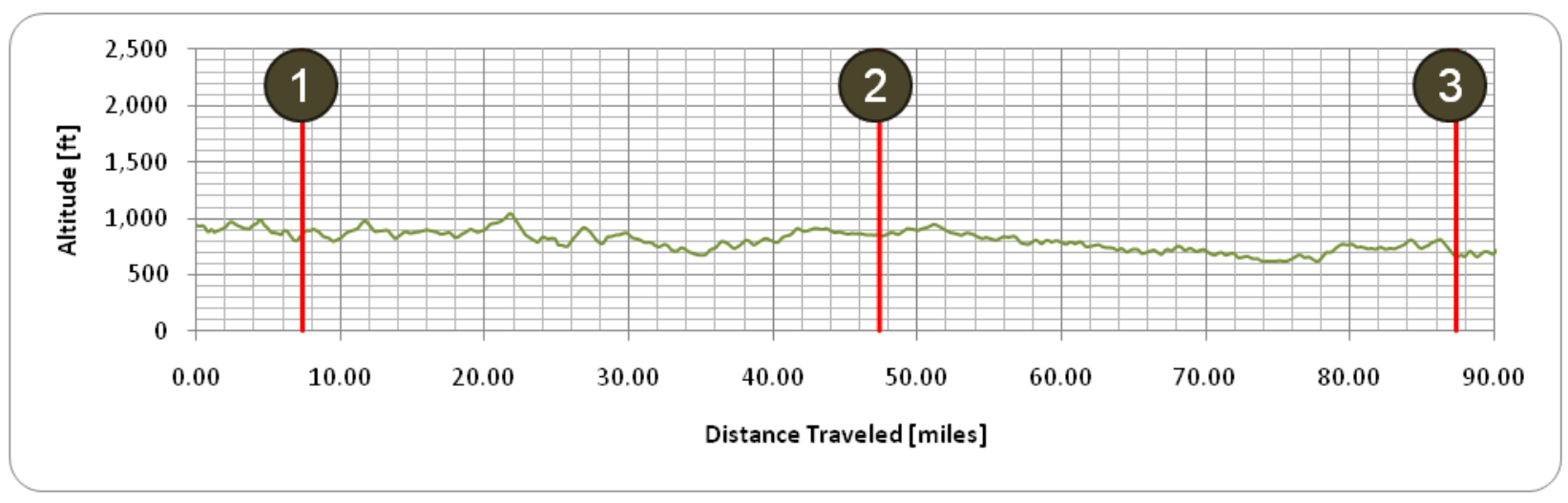

Fig. 6. Knoxville, TN-Cleveland, TN Terrain Profile by Sub-segment.

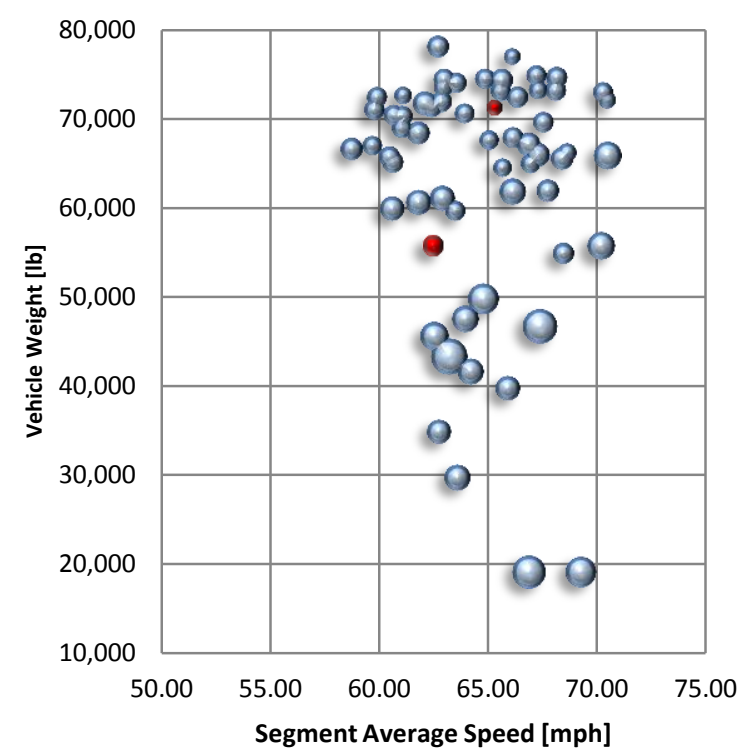

Fig. 7. Knoxville, TN-Cleveland, TN Segment 1-2 FE by Trip Speed and Weight.

(Note: Bubble Size Proportional to FE; Speed Variability: Blue Bubbles <0.15, Red Bubbles 0.15 to 0.50 )

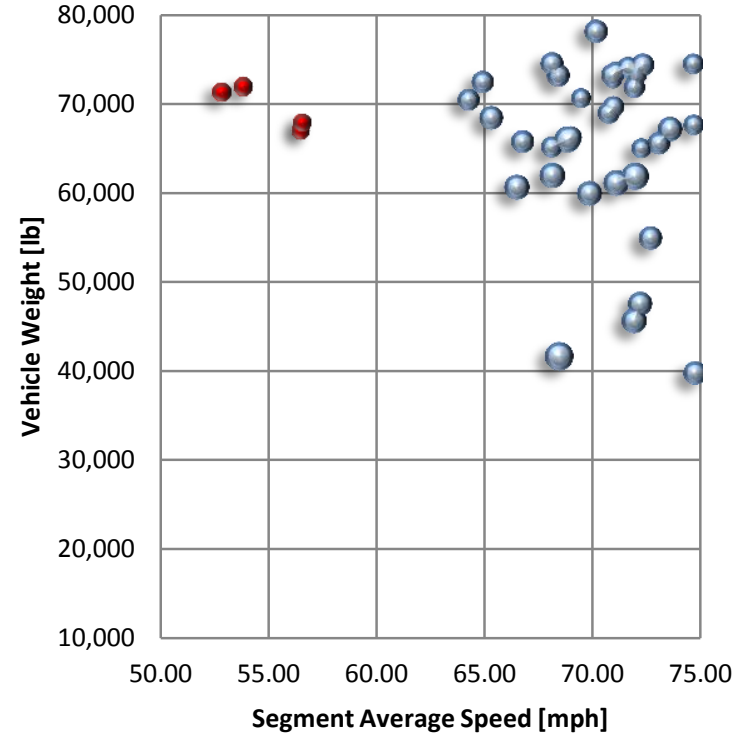

Fig. 8. Knoxville, TN-Cleveland, TN Segment 2-3 FE by Trip Speed and Weight.

(Note: Bubble Size Proportional to FE; Speed Variability: Blue Bubbles <0.15, Red Bubbles 0.15 to 0.50 ) 


\subsubsection{Knoxville, Tennessee to Nashville, Tennessee Segment}

Twenty-eight trips were extracted from the HTDC database which traveled the Knoxville, TN-Nashville, TN corridor. The characteristics of this segment, which was divided into four sub-segments of 40 miles each, are presented in Table 3 . The table shows that the average vehicle weight was approximately $69,000 \mathrm{lb}$ with average sub-segment speeds ranging from $64 \mathrm{mph}$ to $69 \mathrm{mph}$. The sub-segment with the lowest average FE (5.2 mpg) was sub-segment 2-3, and the one with the highest was sub-segment 3-4 (8.9 mph). These two sub-segments included the Cumberland Plateau (see Fig. 9), with sub-segment 2-3 containing the uphill portion reaching towards the crest of the plateau and sub-segment 3-4 the downslope portion coming down to the lower elevations of middle Tennessee (see Fig. 10 and Fig. 13). For each of the four sub-segments and for the 28 trips considered, the speed variability was, in general, low, with only few cases with variability between 0.15 and 0.50 .

Table 3. Knoxville, TN-Nashville, TN Segment Description and General Statistics

\begin{tabular}{|c|c|c|c|c|c|c|c|c|}
\hline \multirow{2}{*}{ Segment } & \multirow{2}{*}{$\begin{array}{l}\text { Segment } \\
\text { Length } \\
\text { [mile] }\end{array}$} & \multirow{2}{*}{$\begin{array}{l}\text { Number } \\
\text { of Trips }\end{array}$} & \multirow{2}{*}{$\begin{array}{l}\text { Average Speed } \\
\text { [mph] }\end{array}$} & \multirow{2}{*}{$\begin{array}{c}\text { Average Vehicle } \\
\text { Weight } \\
\text { [lb] }\end{array}$} & \multirow{2}{*}{$\begin{array}{c}\text { Average FE } \\
\text { [mpg] }\end{array}$} & \multicolumn{3}{|c|}{ Speed Variability [\# of Trips] } \\
\hline & & & & & & $<.15$ & .15 to .50 & $>.50$ \\
\hline $1-2$ & 40.0 & 28 & 63.5 & 68,954 & 6.5 & 25 & 3 & 0 \\
\hline $2-3$ & 40.0 & 28 & 67.6 & 68,954 & 5.2 & 25 & 3 & 0 \\
\hline $3-4$ & 40.0 & 28 & 68.9 & 68,954 & 8.9 & 26 & 2 & 0 \\
\hline 4-5 & 40.0 & 28 & 69.2 & 68,954 & 6.7 & 26 & 2 & 0 \\
\hline
\end{tabular}

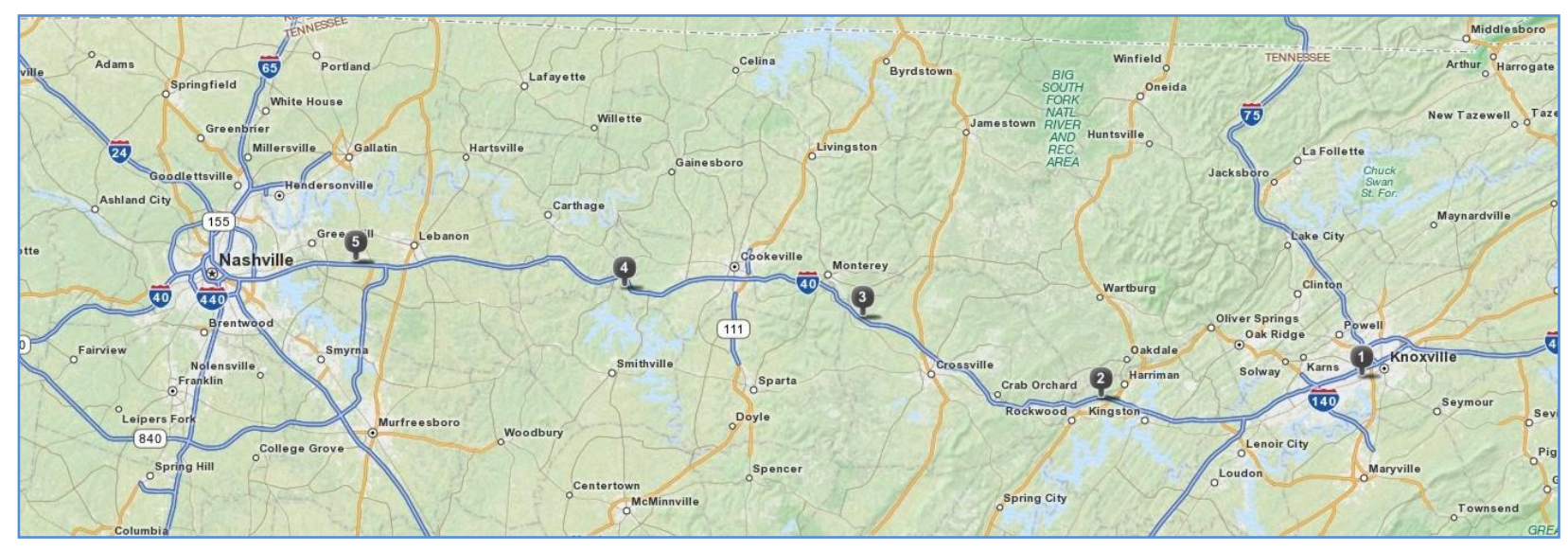

Fig. 9. Knoxville, TN- Nashville, TN Sub-segments.

Fig. 10 and Fig. 13 show the terrain profile for sub-segments 1-2 and 2-3, and sub-segments 3-4 and 4-5, respectively. The results of the analysis are presented in Fig. 11 and Fig. 12 for the first two subsegments and in Fig. 14 and Fig. 15 for the remaining two sub-segments. Notice that the lowest FEs correspond to sub-segment 2-3, the one which includes the climb to the plateau, and the highest FEs to sub-segment 3-4, the one with the downslope section at the end of the plateau. 


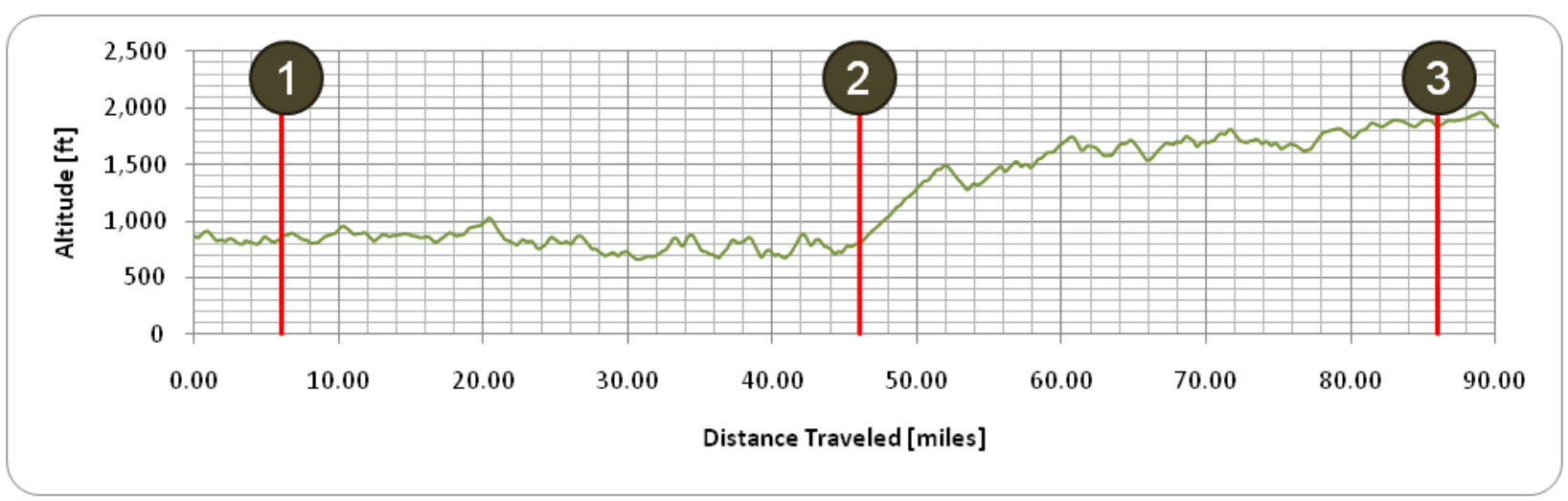

Fig. 10. Knoxville, TN-Nashville, TN Terrain Profile by Sub-segments 1-2 and 2-3.

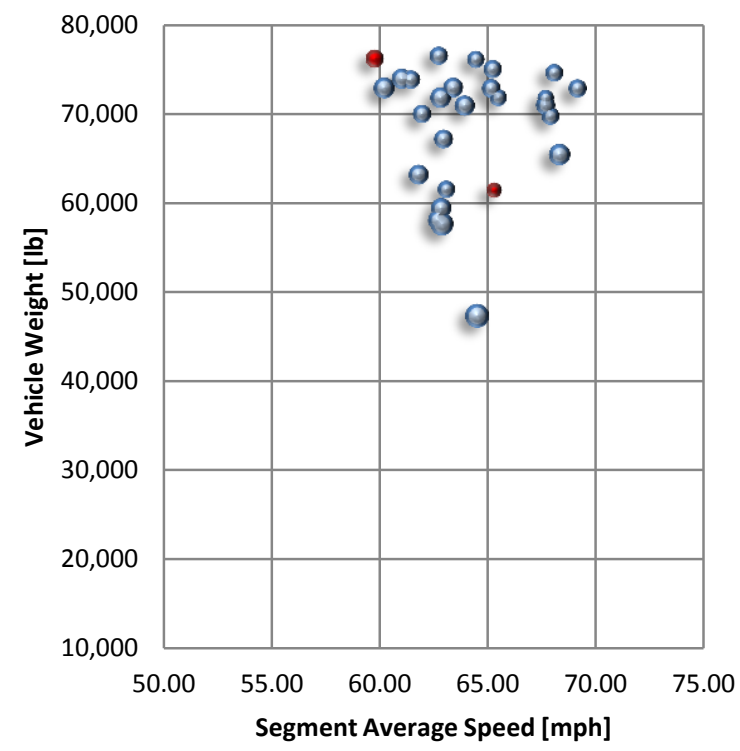

Fig. 11. Knoxville, TN-Nashville, TN Segment 1-2 FE by Trip Speed and Weight.

(Note: Bubble Size Proportional to FE; Speed Variability: Blue Bubbles <0.15, Red Bubbles 0.15 to 0.50 )

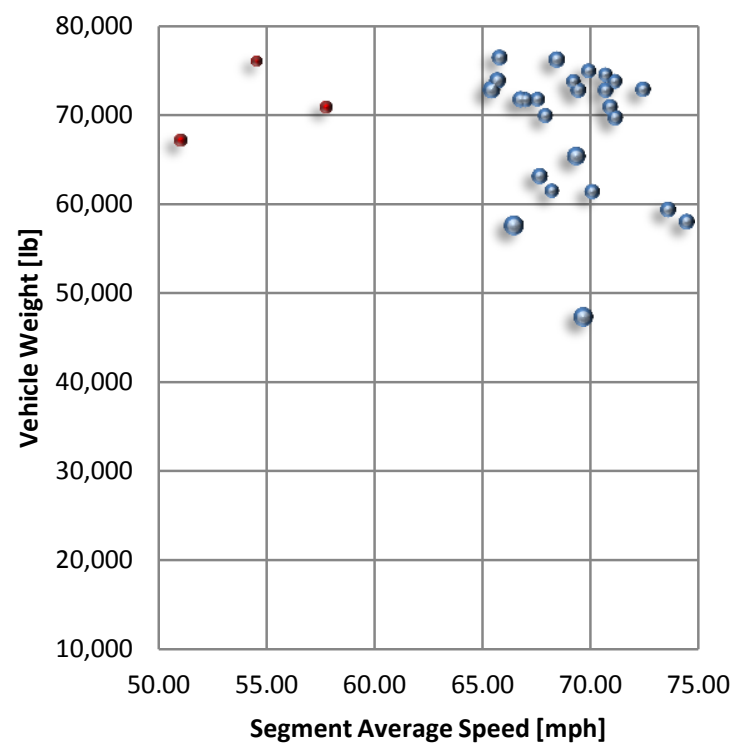

Fig. 12. Knoxville, TN-Nashville, TN Segment 2-3 FE by Trip Speed and Weight.

(Note: Bubble Size Proportional to FE; Speed Variability: Blue Bubbles <0.15, Red Bubbles 0.15 to 0.50 )

Consider, for example, the trip with a vehicle weight of 65,500 lb and average speed of $68 \mathrm{mph}$ in Fig. 11. For this trip the FE decreased from $7.4 \mathrm{mpg}$ (sub-segment 1-2) to $6.3 \mathrm{mpg}$ (sub-segment 2-3). The driver maintained almost identical average speeds for both sub-segments (i.e., $68.3 \mathrm{mph}$ and $69.3 \mathrm{mph}$, respectively) and the same speed variability (0.04), therefore, the decrease in FE (almost $15 \%$ ) can be attributed exclusively to the uphill terrain. For sub-segment 3-4 (downsloping terrain), this trip showed a FE of $10.7 \mathrm{mpg}$ (a gain of almost $49 \%$ with respect to sub-segment 1-2, mostly flat terrain). Finally, for the last sub-segment, the FE was 7.9 mpg (6\% higher than that achieved in sub-segment 1-2, which has a very similar topography). The average speed was very similar for both sub-segments 1-2 and 4-5 (just 1.5 mph higher for the latter) but the speed variability was half for the last sub-segment of the trip when 
compared to the first one (0.02 for sub-segment 4-5 vs. 0.04 for sub-segment 1-2). Although this is just one observation, it shows the effect that congestion (larger speed variability) has on FE.

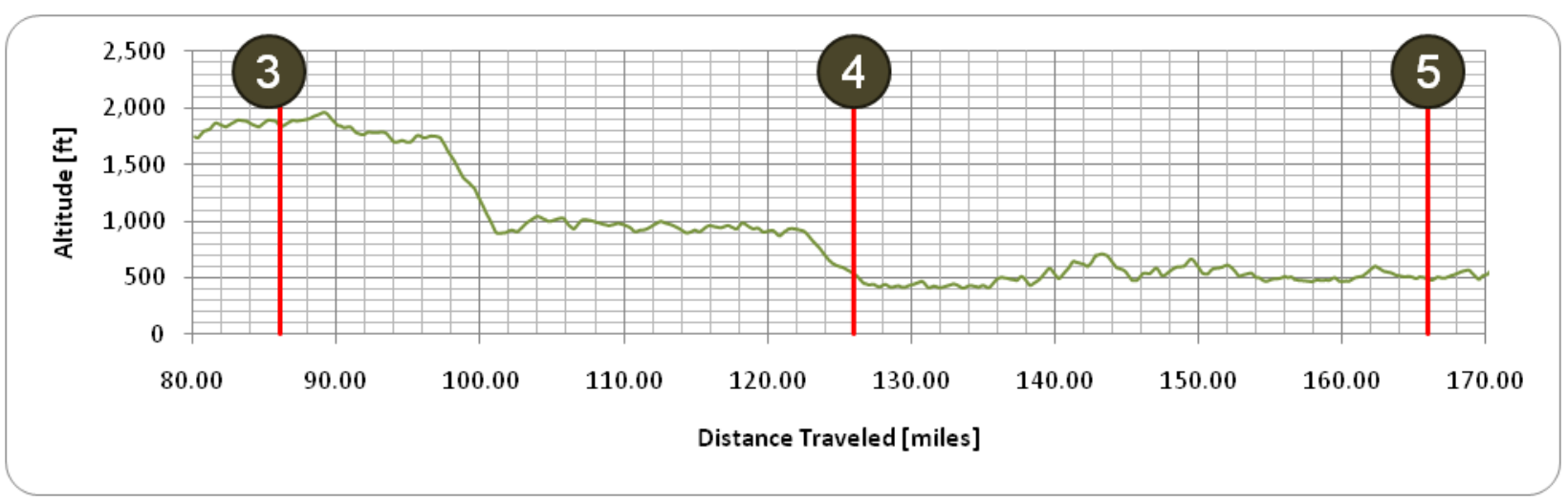

Fig. 13. Knoxville, TN-Nashville, TN Terrain Profile by Sub-segments 3-4 and 4-5.

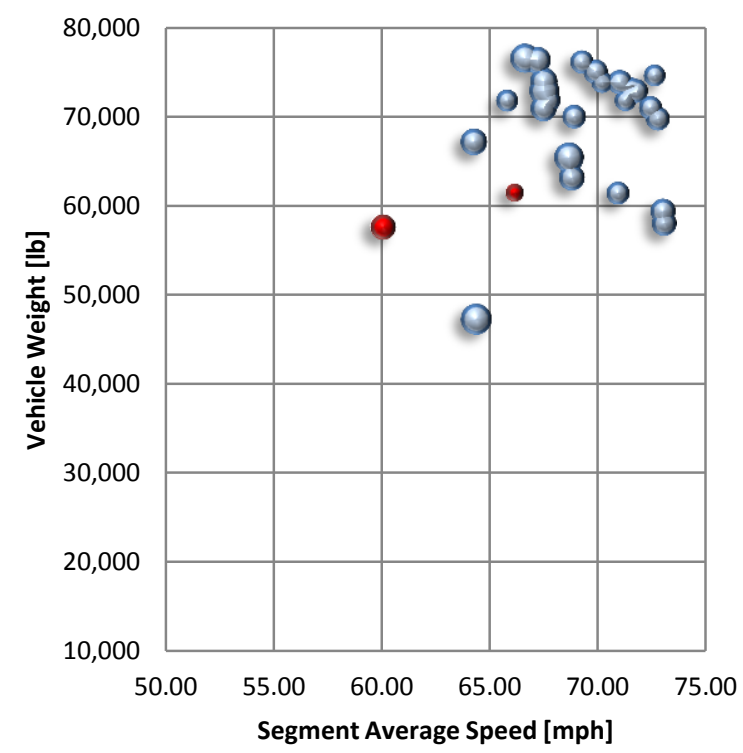

Fig. 14. Knoxville, TN-Nashville, TN Segment 3-4 FE by Trip Speed and Weight.

(Note: Bubble Size Proportional to FE; Speed Variability: Blue Bubbles $<0.15$, Red Bubbles 0.15 to 0.50 )

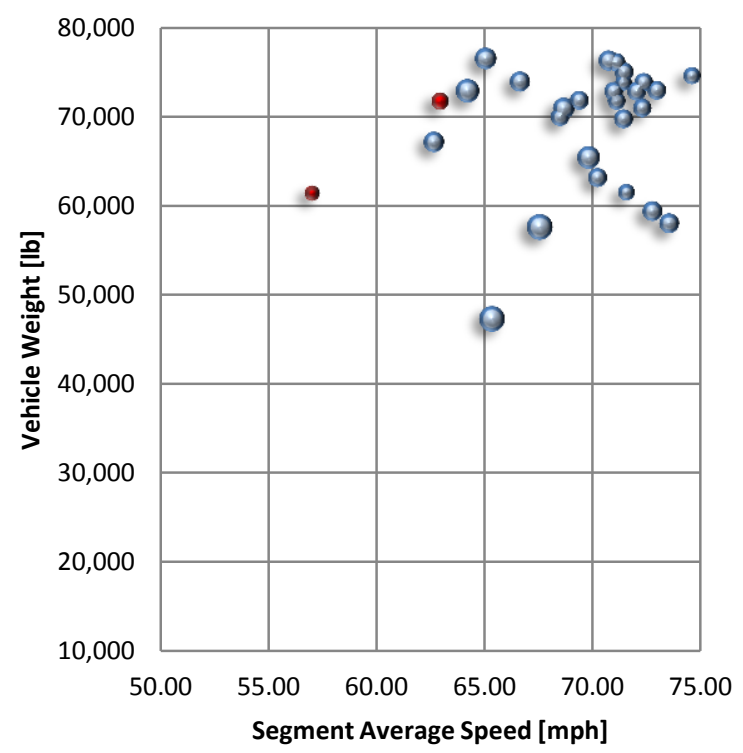

Fig. 15. Knoxville, TN-Nashville, TN Segment 4-5 FE by Trip Speed and Weight.

(Note: Bubble Size Proportional to FE; Speed Variability: Blue Bubbles <0.15, Red Bubbles 0.15 to 0.50 )

\subsubsection{Knoxville, Tennessee to London, Kentucky Segment}

The Knoxville, TN-London, KY segment includes two sub-segments of 40 miles each on I-75 which crosses the Smoky Mountains between the states of Tennessee and Kentucky (see Fig. 16). The database contained 50 trips with an average vehicle weight of 57,000 lb which completely covered these two subsegments. Table 4 shows that the average speed for both segments was almost the same, although subsegment 2-3 had a larger percentage of trips with high speed variability. As expected, and because sub- 
segment 1-2 contained the uphill portion of the trip, the average FE in this sub-segment was very low (5.8 $\mathrm{mpg}$ ) when compared to sub-segment $2-3(8.0 \mathrm{mpg})$, the downhill portion of the trip.

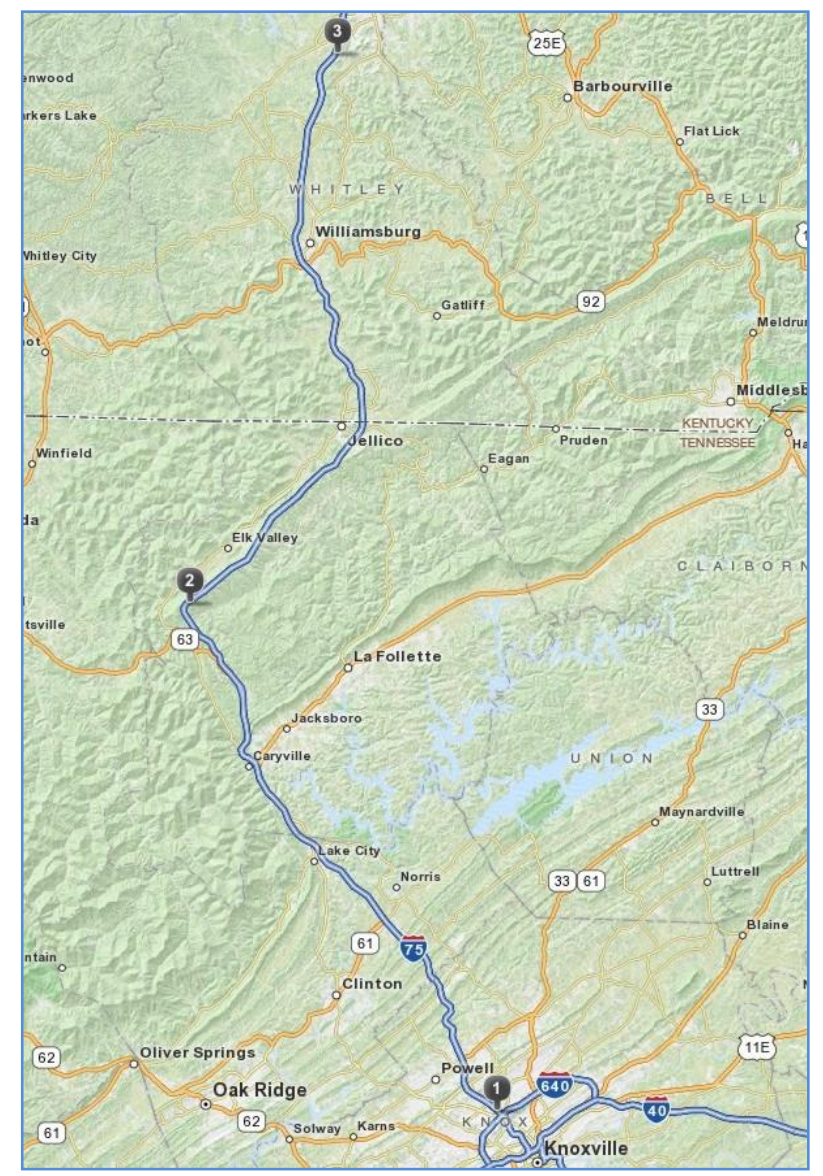

Fig. 16. Knoxville, TN-London, KY Sub-segments.

Table 4. Knoxville, TN-London, KY Segment Description and General Statistics

\begin{tabular}{|c|c|c|c|c|c|c|c|c|}
\hline \multirow{2}{*}{ Segment } & \multirow{2}{*}{$\begin{array}{l}\text { Segment } \\
\text { Length } \\
\text { [mile] }\end{array}$} & \multirow{2}{*}{$\begin{array}{l}\text { Number } \\
\text { of Trips }\end{array}$} & \multirow{2}{*}{$\begin{array}{c}\text { Average Speed } \\
{[\mathrm{mph}]}\end{array}$} & \multirow{2}{*}{$\begin{array}{c}\text { Average Vehicle } \\
\text { Weight } \\
\text { [lb] }\end{array}$} & \multirow{2}{*}{$\begin{array}{c}\text { Average FE } \\
\text { [mpg] }\end{array}$} & \multicolumn{3}{|c|}{ Speed Variability [\# of Trips] } \\
\hline & & & & & & $<.15$ & .15 to .50 & $>.50$ \\
\hline $1-2$ & 40.0 & 50 & 61.7 & 56,969 & 5.8 & 43 & 3 & 4 \\
\hline 1-2 NOL & 40.0 & 56 & 63.4 & 56,329 & 5.8 & 43 & 3 & 4 \\
\hline $2-3$ & 40.0 & 50 & 61.3 & 56,969 & 8.0 & 35 & 5 & 10 \\
\hline 2-3 NOL & 40.0 & 40 & 68.1 & 56,258 & 8.4 & 35 & 5 & 10 \\
\hline
\end{tabular}

The results of the analysis are presented in Fig. 18 and Fig. 19, with Fig. 17 showing the terrain profile for the two sub-segments of the Knoxville, TN-London, KY trip. Notice that since the sub-segment 1-2 contains the steepest sub-segment of all the ones analyzed in this section, it also presents the smaller FEs (see Fig. 18). Notice also that between the uphill and downhill sub-segments the difference in FE is larger as the vehicle weight increases. However, for very low vehicle weights (e.g., the tractor only trip shown in Fig. 18 and Fig. 19), there is almost no difference in FE between the two sub-segments (10.8 mpg in sub-segment 1-2 vs. 11.3 mpg in sub-segment 2-3). 


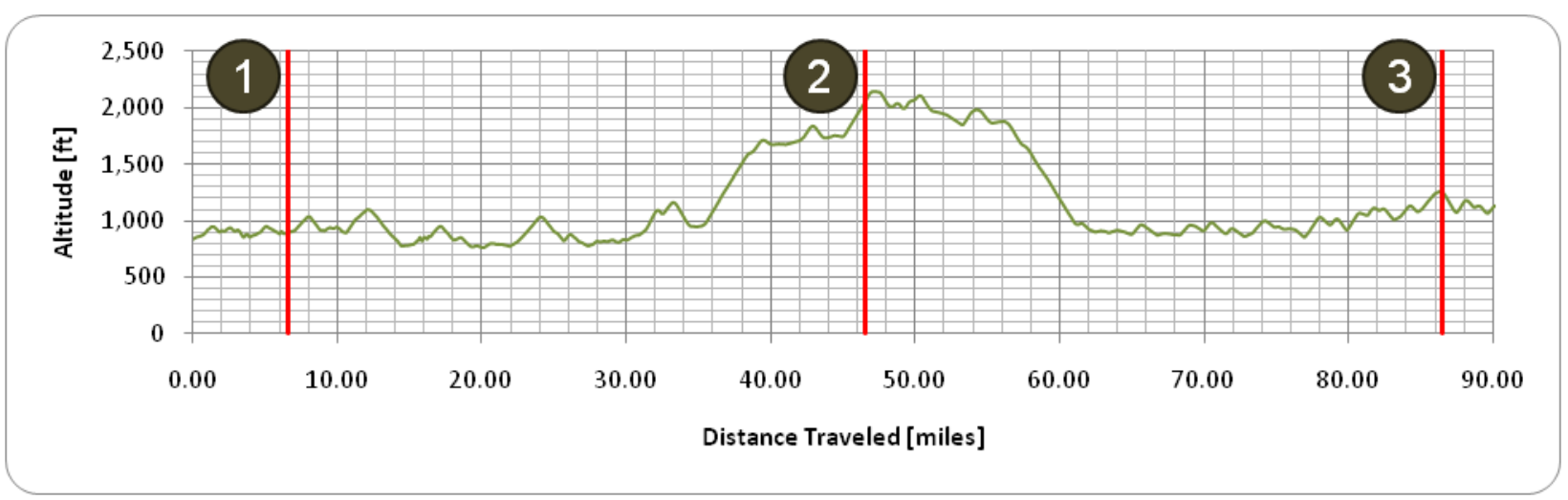

Fig. 17. Knoxville, TN-London, KY Terrain Profile by Sub-segment.

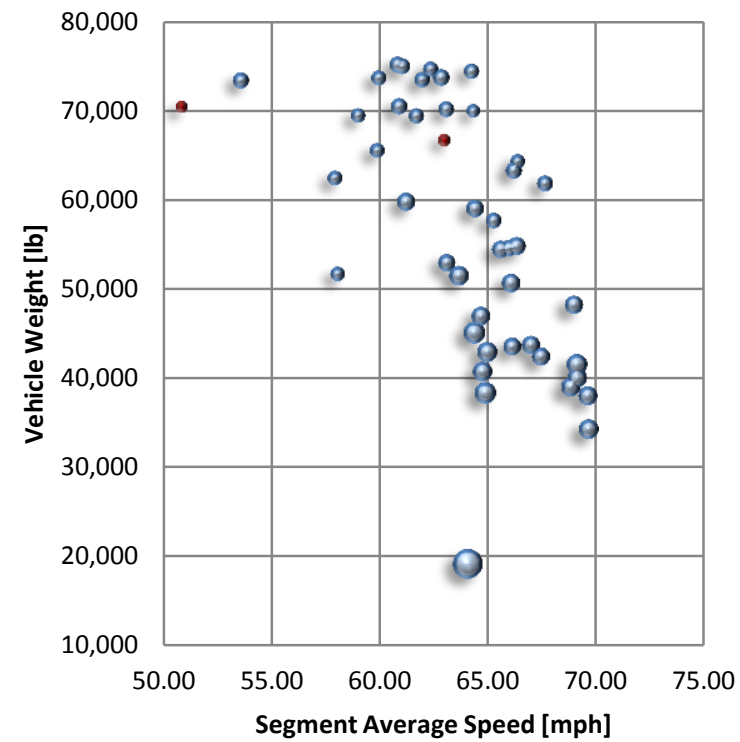

Fig. 18. Knoxville, TN-London, KY Segment 1-2 FE by Trip Speed and Weight.

(Note: Bubble Size Proportional to FE; Speed Variability: Blue Bubbles <0.15, Red Bubbles 0.15 to 0.50 )

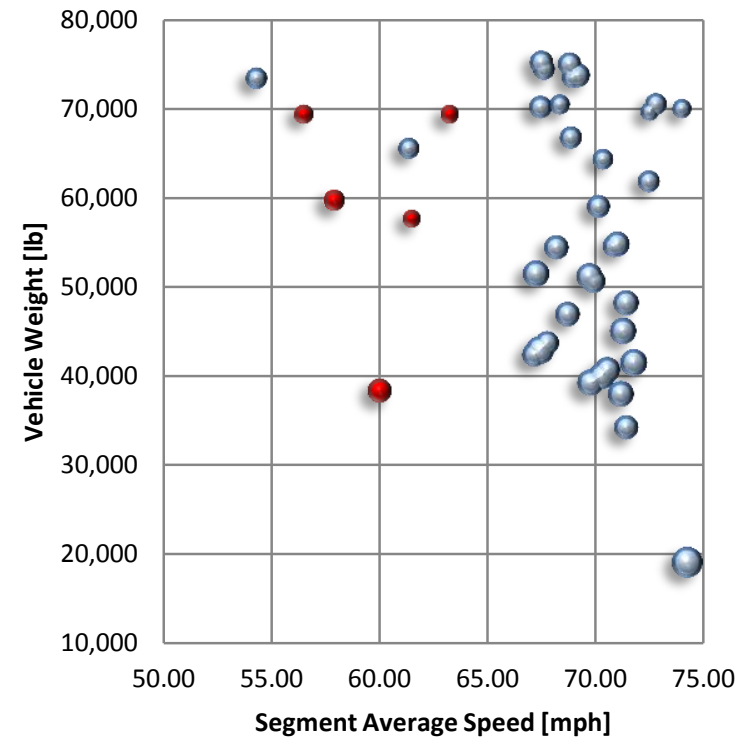

Fig. 19. Knoxville, TN-London, KY Segment 2-3 FE by Trip Speed and Weight.

(Note: Bubble Size Proportional to FE; Speed Variability: Blue Bubbles <0.15, Red Bubbles 0.15 to 0.50)

\subsection{SUMMARY AND CONCLUSSIONS}

Three segments of Interstate freeways, divided into 40-mile long sub-segments, were considered for the analysis of the effect that the type of terrain, speed, and vehicle weight has on the FE of Class- 8 trucks. Two of the three factors (i.e., terrain type and vehicle speed) were aggregated over the length of each subsegment. A total of 140 trips were analyzed and three type of terrains were considered: flat terrain (62 trips); flat-upslope-downslope terrain (28 trips); and rolling-upslope-downslope terrain (50 trips). In general, and as expected, FE decreases as the total vehicle weight increases. The terrain also has a very important effect, with uphill terrains severely decreasing FEs. Regarding speed, FE increases as speed increases and then it starts to decrease again. The speed variability (speed standard deviation divided by the average speed in any given sub-segment) which is a proxy for congestion, has a negative effect on FE. 


\section{EFFECT OF VEHICLE SPEED AND PAYLOAD ON FUEL EFFICIENCY - FLAT TERRAIN}

The previous section presented an analysis of the effect that the roadway grade (i.e., terrain profile) and the vehicle speed and weight have on the FE of Class- 8 trucks. The study was performed by considering long freeway segments (i.e., 40 miles in length) and comparing the FEs of all the trips that traveled each particular segment as a function of the vehicle weight and the average speed. The effect of the type of terrain was analyzed by comparing segments that had different terrain profiles.

Another approach to study the effect that roadway grade, vehicle speed, and vehicle weight have on the FE of large trucks is by parsing the information contained in the HTDC database (mainly fuel consumption and distance traveled) into a set of bins for vehicle speed, vehicle weight, and roadway grade. This is the approach taken in this and the next chapters of this report. The roadway grade was divided into 7 bins, going from grades below $-5 \%$ (very rare on freeways, except for freeways traversing the Rocky Mountains), to grades between $-5 \%$ and $-3 \%$ (severe down slope), $-3 \%$ to $-1 \%$ (mild down slope), $-1 \%$ to $1 \%$ (flat terrain), $1 \%$ to $3 \%$ (mild upslope), $3 \%$ to $5 \%$ (severe upslope), and grades above $5 \%$ (also very rare on freeways). The vehicle speed was divided into $1 \mathrm{mph}$ and $2 \mathrm{mph}$ bins, and the vehicle weight into 2,500 lb and 5,000 lb bins. The procedure used was as follows. For each trip in the HTDC database and for each record of that trip (collected once every .2 seconds), the roadway grade was used to determine on which of the 7 roadway grade bins the vehicle was traveling. The distance $\left(d_{l}\right)$ that the vehicle traveled in the .2 second interval was recorded and assigned to the corresponding roadway grade bin. Then the next record in the database was processed, and if the roadway grade bin was the same as the last one, the newly computed distance $d_{2}$ was added to the last one (i.e., the distance traveled under this type of terrain was accumulated). This procedure continued until either the roadway-grade bin changed or a preset travel distance of a $1 / 4$ mile was reached. If the roadway-grade bin changed, then the accumulated distance was set to 0 and the process started again. If, on the other hand, the preset travel distance was reached, then that distance was also set to $0^{2}$ but as new records were read that corresponded to the same roadway grade bin, the distance traveled as well as the fuel consumed were accumulated for the corresponding roadway grade bin, vehicle speed bin, and vehicle weight bin. When the grade bin changed, then the process started over.

Using the information collected this way, the next section analyzes the effect that speed has on FE for different vehicle weigh levels when the vehicle was traveling on flat terrain ${ }^{3}$. The following section focuses on the effect that the vehicle weight has on the FE while traveling at different speed ranges. In that section of this chapter, an extrapolation of FE as a function of vehicle weight for the prevalent freeway speed limit (i.e., $65 \mathrm{mph}$ ) is also presented. In the last section of this chapter, the two variables, vehicle speed and vehicle weight are combined in a three-dimensional graph of $\mathrm{FE}$ as a function of these two variables.

\footnotetext{
${ }^{2}$ All the information collected in the first $1 / 4$ of a mile while a vehicle was traveling on a given type of terrain was discarded to avoid the "contamination" resulting from driving conditions imposed by the immediately upstream roadway segment that had a different type of terrain. See the first chapter of this report for a more detailed explanation.

${ }^{3}$ More than $50 \%$ of the distance traveled by the vehicles participating in this study was on terrains that had roadway grades between $-1 \%$ and $1 \%$, i.e., flat terrain.
} 


\subsection{EFFECT OF VEHICLE SPEED ON FUEL EFFICIENCY - FLAT TERRAIN}

Once all the records in the HTDC database were processed as explained above, the result was a series of distances and fuel consumed under different terrain types, speed, and vehicle weight conditions, which allowed calculating the FE of the vehicles while traveling under these conditions. Fig. 20 shows the distance traveled and fuel consumed while the vehicles in the HTDC database were traveling on flat terrain (-1\% to $1 \%$ roadway grade) aggregated across all the vehicle weight bins. Notice that the graphs have two peaks. This was due to the fact that during the one-year data collection period (2006-07), the trucking company participating in the study implemented a policy by which their vehicles could not be accelerated above $68 \mathrm{mph}$ (i.e., approximately seven months into the project they installed a speed governor in all their tractors). The vehicle could travel at higher speeds (e.g., on a downhill terrain), but the driver could not achieve speeds higher $68 \mathrm{mph}$ by pressing the accelerator pedal. Notice also that there is almost no distance traveled at speeds lower than $55 \mathrm{mph}$. The reason for this is that the trips were mostly long-haul trips and the vehicles traveled a very high proportion of each trip on freeways using arterials and surface streets only at both ends of the trip. Fig. 21 shows the same information as Fig. 20, but considering only trips for which both tractor and trailer had AirWeigh sensors.

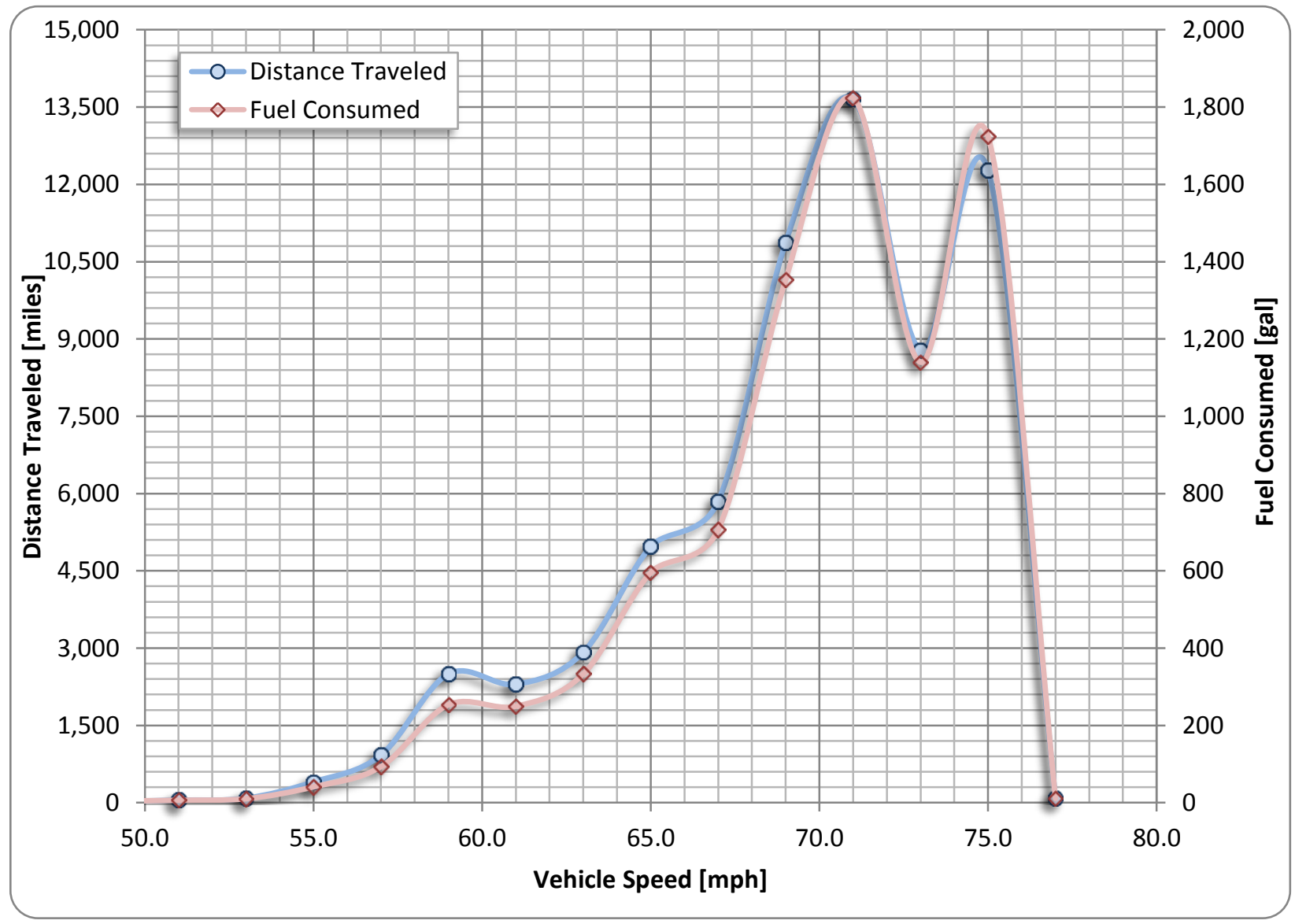

Fig. 20. Total Distance Traveled and Total Fuel Consumed vs. Vehicle Speed - Flat Terrain. 


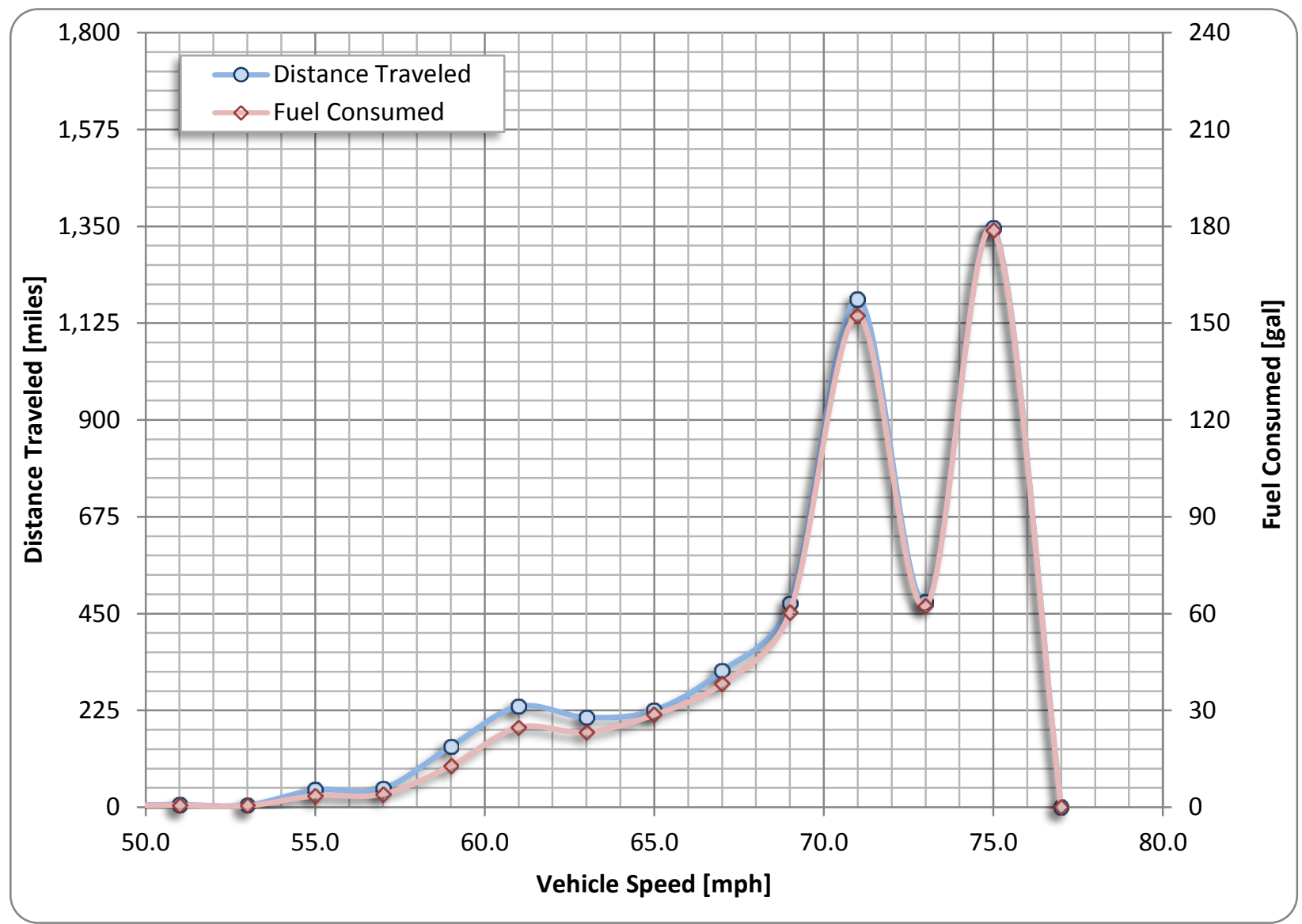

Fig. 21. Total Distance Traveled and Total Fuel Consumed vs. Vehicle Speed - Flat Terrain, Tractor and Trailer with Weigh Sensors.

In Fig. 20 (and also in Fig. 21) both the total distance traveled and the total fuel consumed as a function of the vehicle speed were scaled in such a way that the two maximum coincide. For example, Fig. 20 shows that most of the miles traveled and also most of the fuel consumed was at about $71 \mathrm{mph}$. Scaled this way, the differences between the two graphs show speeds at which FE was better (distance-traveled line above fuel-consumed line, mostly speeds below $70 \mathrm{mph}$ ) and worse (distance-traveled line below fuel-consumed line, mostly speeds above $73 \mathrm{mph}$ ).

This can also be observed in Table 5 and Table 6 which show FE information as a function of speed for vehicle weight levels of 60,000 lb-70,000 lb and 70,000 lb-80,000 lb, respectively. Consider, for example, Table 5. For vehicles hauling between $60,000 \mathrm{lb}$ and 70,000 lb, the table shows for any given speed $^{4}$ the total distance traveled at that speed and the total fuel consumed. The FE column then shows the ratio between these two quantities, but it is only computed when the travel distance is at least 10 miles for that speed. The last column in the table shows the average weight of the vehicles that "contributed" to the distance traveled and fuel consumed showed in that line. Notice that while traveling on flat terrain, in both tables the optimal speed (i.e., the speed at which the maximum FE is achieved) is about 58-59 mph.

\footnotetext{
${ }^{4}$ The speed shown in the table is the center of the speed interval. For example, $59 \mathrm{mph}$ indicates speeds that can range between $58 \mathrm{mph}$ and $60 \mathrm{mph}$.
} 
Table 5. Fuel Efficiency by Speed for Flat Terrain Vehicle Weight 60,000 lb to 70,000 lb

\begin{tabular}{rrrrr}
\hline $\begin{array}{c}\text { Speed } \\
\text { [mph] }\end{array}$ & $\begin{array}{c}\text { Distance } \\
\text { Traveled } \\
\text { [miles] }\end{array}$ & $\begin{array}{c}\text { Fuel } \\
\text { Consumed } \\
\text { [gal] }\end{array}$ & $\begin{array}{c}\text { FE } \\
\text { [mpg] }\end{array}$ & $\begin{array}{c}\text { Average } \\
\text { Vehicle } \\
\text { Weight [lb] }\end{array}$ \\
\hline $\mathbf{4 3 . 0}$ & 0 & 0 & $*$ & 62,273 \\
\hline $\mathbf{4 5 . 0}$ & 2 & 0 & $*$ & 64,553 \\
\hline $\mathbf{4 7 . 0}$ & 9 & 1 & $*$ & 66,635 \\
\hline $\mathbf{4 9 . 0}$ & 9 & 2 & $*$ & 66,116 \\
\hline $\mathbf{5 1 . 0}$ & 18 & 3 & 5.8 & 66,561 \\
\hline $\mathbf{5 3 . 0}$ & 30 & 4 & 7.6 & 66,923 \\
\hline $\mathbf{5 5 . 0}$ & 149 & 17 & 8.9 & 66,517 \\
\hline $\mathbf{5 7 . 0}$ & 186 & 21 & 8.9 & 65,550 \\
\hline $\mathbf{5 9 . 0}$ & 703 & 72 & 9.8 & 66,249 \\
\hline $\mathbf{6 1 . 0}$ & 695 & 79 & 8.8 & 65,478 \\
\hline $\mathbf{6 3 . 0}$ & 910 & 111 & 8.2 & 65,825 \\
\hline $\mathbf{6 5 . 0}$ & 1,509 & 190 & 7.9 & 66,329 \\
\hline $\mathbf{6 7 . 0}$ & 1,869 & 239 & 7.8 & 66,457 \\
\hline $\mathbf{6 9 . 0}$ & 3,218 & 413 & 7.8 & 66,690 \\
\hline $\mathbf{7 1 . 0}$ & 3,244 & 451 & 7.2 & 66,103 \\
\hline $\mathbf{7 3 . 0}$ & 1,595 & 214 & 7.4 & 66,470 \\
\hline $\mathbf{7 5 . 0}$ & 2,559 & 363 & 7.1 & 67,220 \\
\hline $\mathbf{7 7 . 0}$ & 4 & 0 & $*$ & 65,822 \\
\hline
\end{tabular}

*Not computed because the distance traveled was less than 10 miles.

Table 6. Fuel Efficiency by Speed for Flat Terrain Vehicle Weight 70,000 lb to 80,000 lb

\begin{tabular}{rrrrr}
\hline $\begin{array}{c}\text { Speed } \\
\text { [mph] }\end{array}$ & $\begin{array}{r}\text { Distance } \\
\text { Traveled } \\
\text { [miles] }\end{array}$ & $\begin{array}{c}\text { Fuel } \\
\text { Consumed } \\
\text { [gal] }\end{array}$ & $\begin{array}{c}\text { FE } \\
\text { [mpg] }\end{array}$ & $\begin{array}{c}\text { Average } \\
\text { Vehicle } \\
\text { Weight [lb] }\end{array}$ \\
\hline $\mathbf{4 3 . 0}$ & 1 & 0 & $*$ & 73,278 \\
\hline $\mathbf{4 5 . 0}$ & 4 & 0 & $*$ & 73,137 \\
\hline $\mathbf{4 7 . 0}$ & 5 & 1 & $*$ & 73,924 \\
\hline $\mathbf{4 9 . 0}$ & 11 & 2 & 5.7 & 73,564 \\
\hline $\mathbf{5 1 . 0}$ & 12 & 2 & 7.9 & 73,269 \\
\hline $\mathbf{5 3 . 0}$ & 22 & 3 & 6.7 & 73,206 \\
\hline $\mathbf{5 5 . 0}$ & 55 & 7 & 7.9 & 72,981 \\
\hline $\mathbf{5 7 . 0}$ & 210 & 24 & 8.8 & 73,006 \\
\hline $\mathbf{5 9 . 0}$ & 587 & 68 & 8.7 & 73,351 \\
\hline $\mathbf{6 1 . 0}$ & 610 & 75 & 8.1 & 73,454 \\
\hline $\mathbf{6 3 . 0}$ & 858 & 106 & 8.1 & 73,281 \\
\hline $\mathbf{6 5 . 0}$ & 1,528 & 193 & 7.9 & 73,374 \\
\hline $\mathbf{6 7 . 0}$ & 1,713 & 219 & 7.8 & 73,415 \\
\hline $\mathbf{6 9 . 0}$ & 4,441 & 580 & 7.7 & 73,380 \\
\hline $\mathbf{7 1 . 0}$ & 5,065 & 722 & 7.0 & 73,604 \\
\hline $\mathbf{7 3 . 0}$ & 3,190 & 445 & 7.2 & 73,364 \\
\hline $\mathbf{7 5 . 0}$ & 6,087 & 895 & 6.8 & 74,000 \\
\hline $\mathbf{7 7 . 0}$ & 10 & 1 & 9.8 & 74,906 \\
\hline$* 200$
\end{tabular}

*Not computed because the distance traveled was less than 10 miles. 
Fig. 24 and Fig. 25 show, in graphical form, the same information that is presented in Table 5 and Table 6, while Fig. 22 and Fig. 23 present FE as a function of speed for vehicle weights between 20,000 lb$40,000 \mathrm{lb}$ and 40,000 lb $-60,000 \mathrm{lb}$, respectively. In all of the four figures, the raw data was fitted with a polynomial function of third order (shown in the figures), with coefficients of determination $\mathrm{R}^{2}$ of 0.97 , 0.95, 0.87, and 0.87 for the lines shown in Fig. 22 to Fig. 25, respectively.

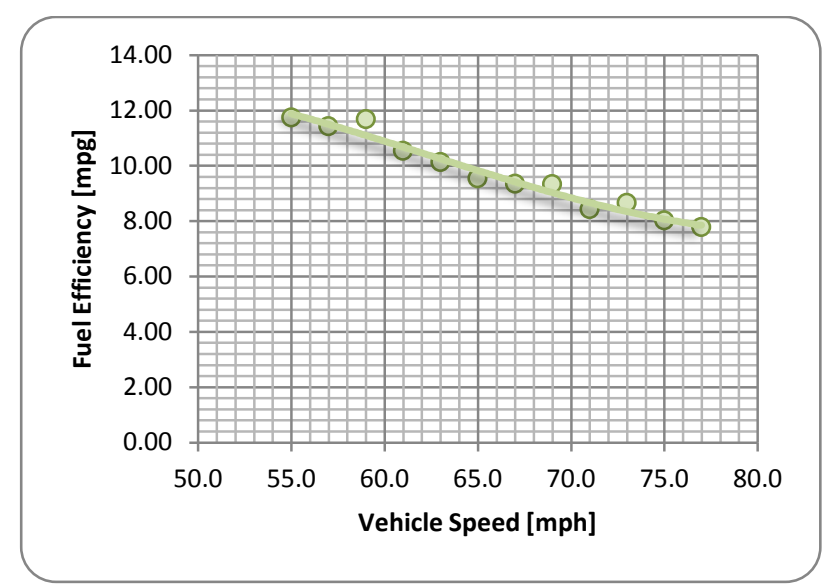

Fig. 22. FE vs. Speed, Vehicle Weight between 20,000 lb to 40,000 lb (Average: 33,700 lb). Flat Terrain.

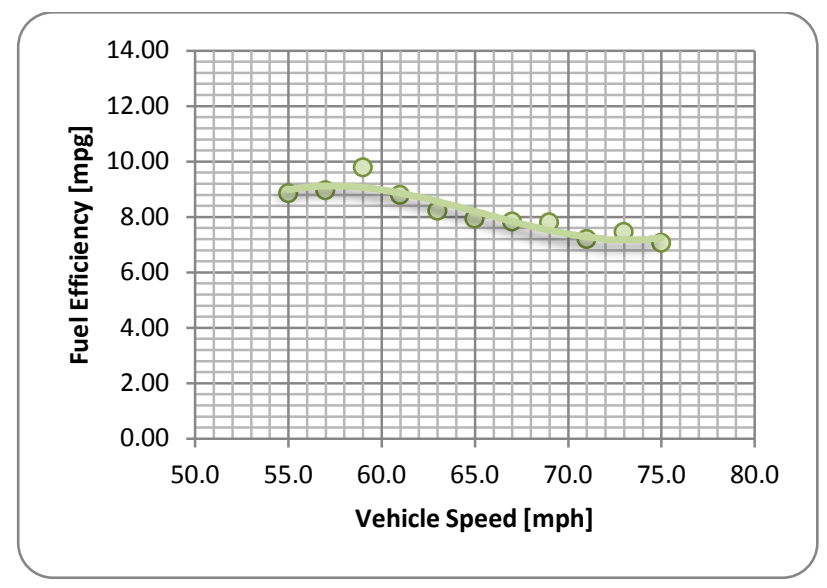

Fig. 24. FE vs. Speed, Vehicle Weight between 60,000 lb to 70,000 lb (Average: 66,400 lb). Flat Terrain.

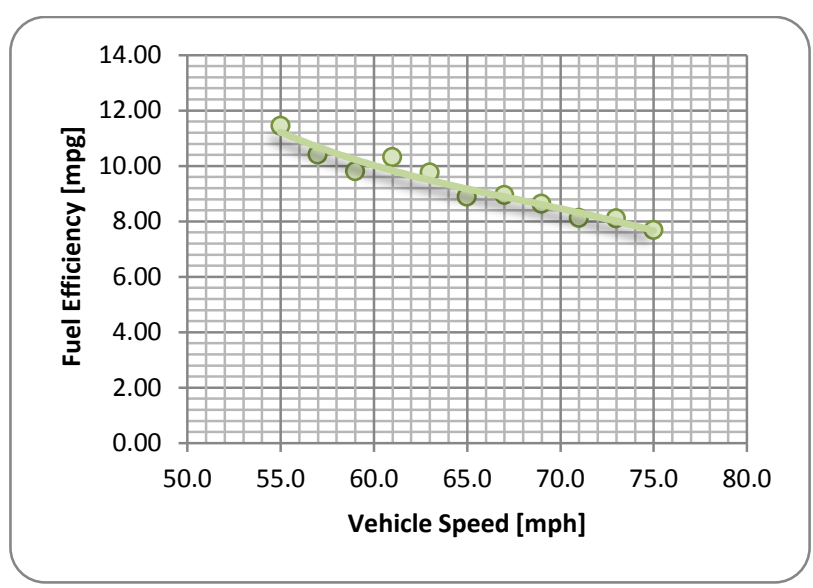

Fig. 23. FE vs. Speed, Vehicle Weight between 40,000 lb to 60,000 lb (Average: 49,700 lb). Flat Terrain.

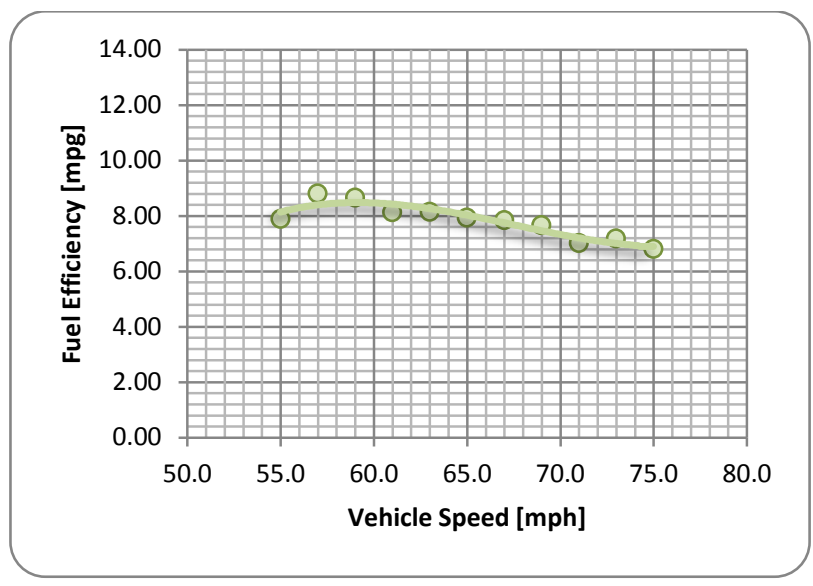

Fig. 25. FE vs. Speed, Vehicle Weight between 70,000 lb to 80,000 lb (Average: 73,600 lb). Flat Terrain.

In order to simplify the analysis, the information shown in Fig. 22 to Fig. 25 was combined into one graph presented in Fig. 26. The graph shows that for light and medium loads (less than 60,000 lbs) the FE decreases almost in a linear fashion as the vehicle speed increases. For heavy loads, this effect does not appear to be linear, but rather there is an increase in FE with speed up to a certain limit (about 58-59 $\mathrm{mph}$ ) after which the FE starts to decrease with increasing vehicle speeds. Notice that, as expected, at any given speed, the FE decreases as the vehicle weight increases. However, this difference appears to decrease with increasing vehicle speeds. 


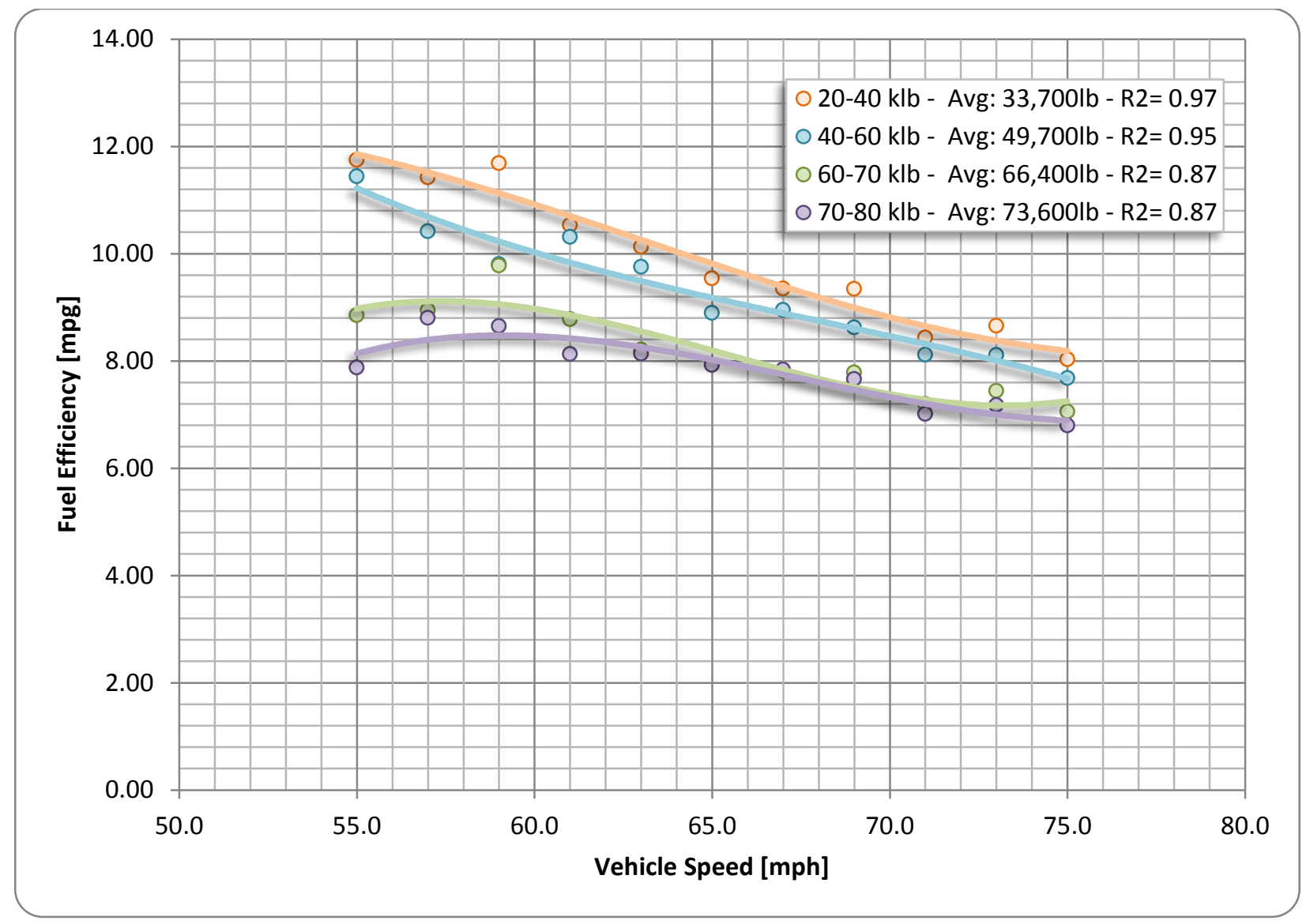

Fig. 26. FE vs. Vehicle Speed for Different Vehicle Weight Levels (with Polynomial Regression Lines). Flat Terrain.

\subsubsection{Trips with Tractor and Trailer Weigh Sensors}

The HTDC database contains about $6 \%$ of trips (roughly 45,000 miles out of the 700,000 miles collected) for which both tractor and trailer were instrumented with AirWeigh sensors. This permitted to determine the vehicle weight by directly reading the information provided by these sensors ${ }^{5}$. The same procedure discussed at the beginning of the chapter was used to parse the information collected when both tractor and trailer were mounted with weight sensors. However, because the total distance traveled under this condition was comparatively small, and the applied procedure discarded the first $1 / 4$ of a mile of any given terrain segment of the seven types considered, the size of useful dataset was greatly reduced. Moreover, only accumulated distances of more than 10 miles were used to compute FE, which further decreased the size of the dataset. Because of this constraint, only speeds above $60 \mathrm{mph}$ were considered (i.e., there was not enough information for speeds lower than $60 \mathrm{mph}$ to reliably compute FE values).

Fig. 27 presents the FE vs. vehicle speed data for different vehicle weight levels using only information collected when both tractor and trailer were mounted with weight sensors. Comparing the information

\footnotetext{
${ }^{5}$ For the remaining $94 \%$ of the trips, only the tractor was mounted with AirWeigh sensors. ORNL developed and applied weight models that used the laws of physics to predict the weight of the trailer based on the readings provided by the tractor weight sensors. More information on these models can be found in [5].
} 
presented in that figure with the one shown in Fig. 28 (same as Fig. 26, but constrained to vehicle speeds ranging from $60 \mathrm{mph}$ to $75 \mathrm{mph}$ ), it is possible to see that both datasets show the same tendencies regarding the effects that speed and weight have on FE, although with more variability in Fig. 27 due to the much smaller data sampling size (specially at speeds in the lower part of the range, were the traveled distances were not very large).

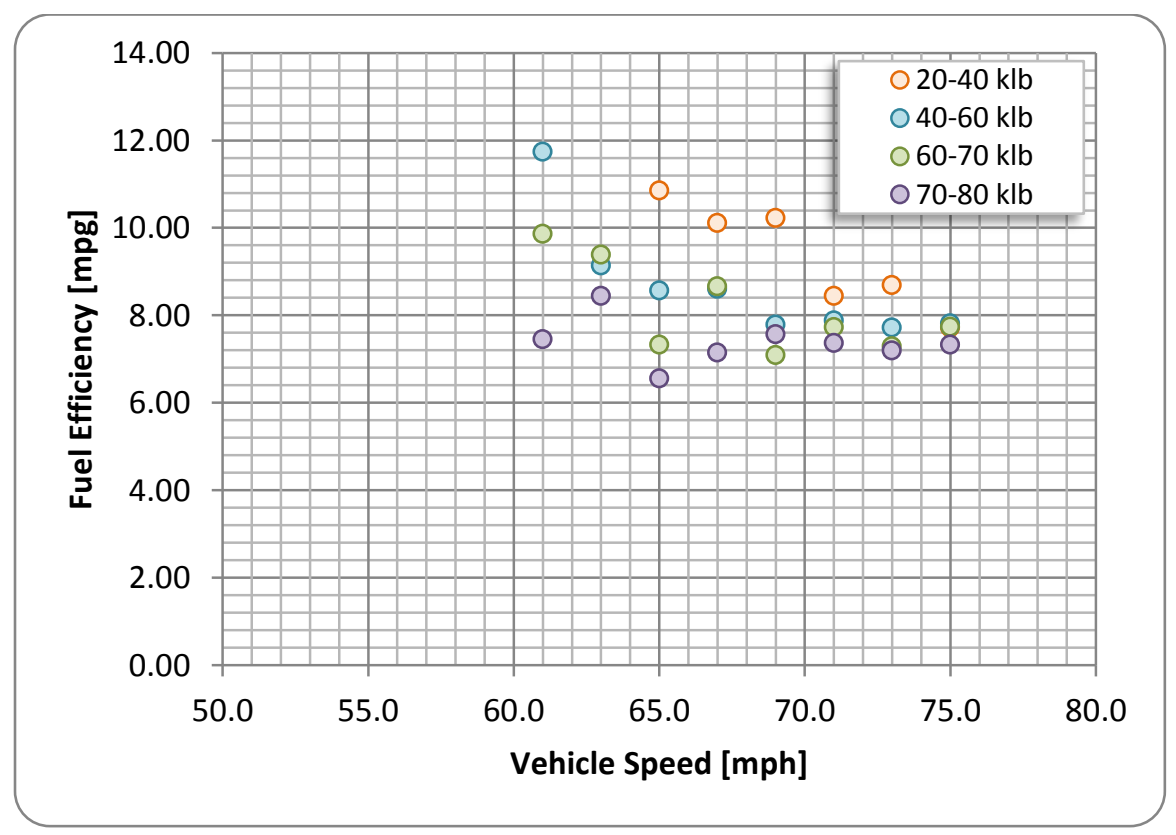

Fig. 27. FE vs. Vehicle Speed for Different Vehicle Weight Levels. Trips with Tractor and Trailer Weight Sensors - Flat Terrain.

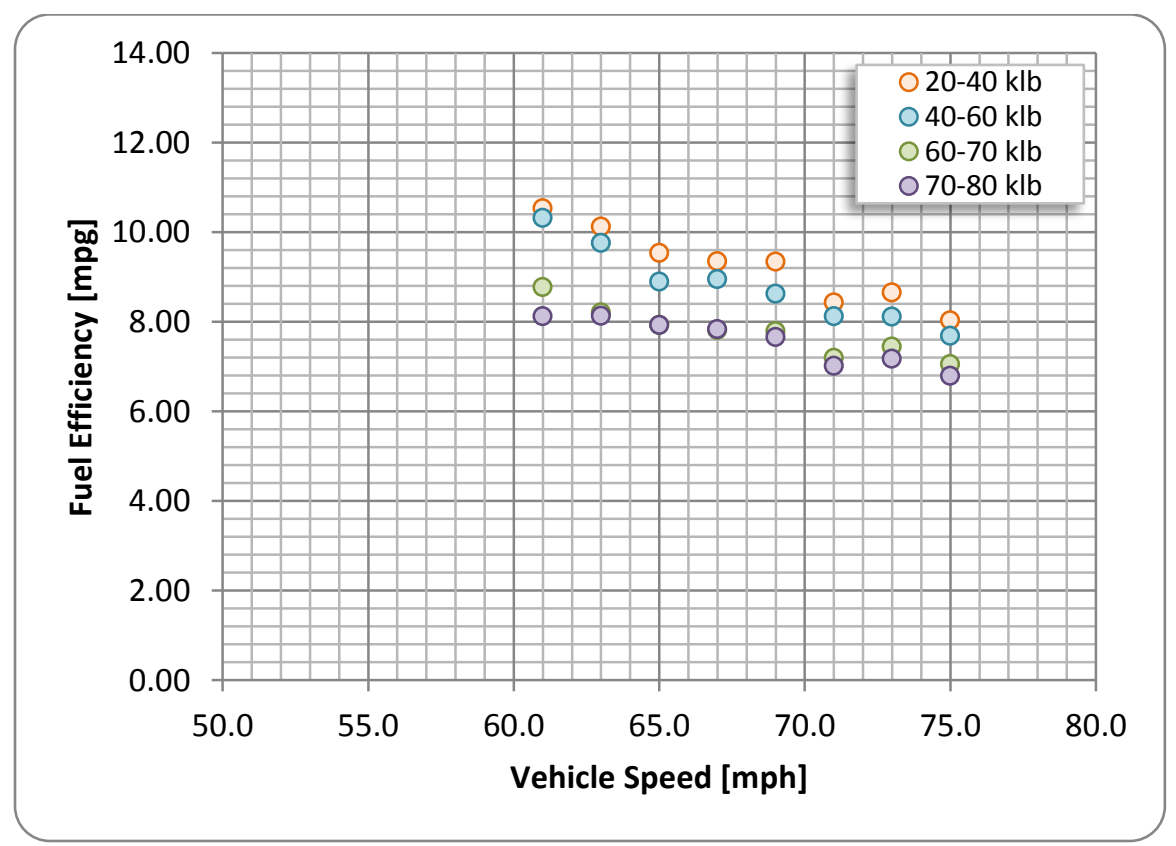

Fig. 28. FE vs. Vehicle Speed for Different Vehicle Weight Levels. All Trips -Flat Terrain. 


\subsection{EFFECT OF VEHICLE WEIGHT ON FUEL EFFICIENCY - FLAT TERRAIN}

The processed flat-terrain data was also aggregated across speed bins to study the effect that the vehicle weight has on FE. Fig. 29 to Fig. 32 present the FE vs. vehicle weight for speed ranges 55-60 mph, 60$65 \mathrm{mph}, 65-70 \mathrm{mph}$, and 70-75 $\mathrm{mph}$, respectively. The figures include the raw data and polynomial fitting curves similar to those used to fit the FE vs. vehicle speed data (see Fig. 22 to Fig. 25) but of second degree instead of third degree. In this case, and as it can be seen in the pictures, the data had more variability and therefore these fitting curves had a lower $\mathrm{R}^{2}$ than in the case of FE vs. vehicle speed (the $\mathrm{R}^{2}$ ranged from 0.53 to 0.61 ).

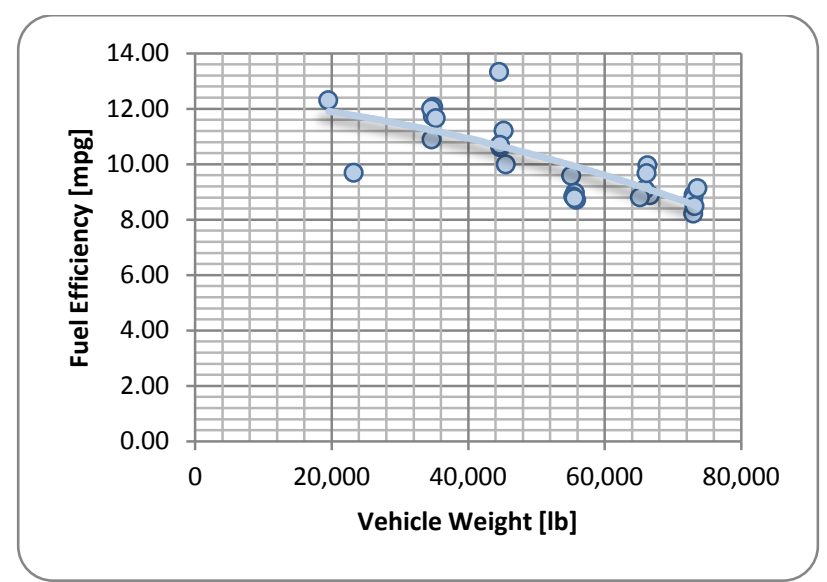

Fig. 29. FE vs. Vehicle Weight, Vehicle Speed between $55 \mathrm{mph}$ to $60 \mathrm{mph}$ (Average: $58.3 \mathrm{mph}$ ).

Flat Terrain.

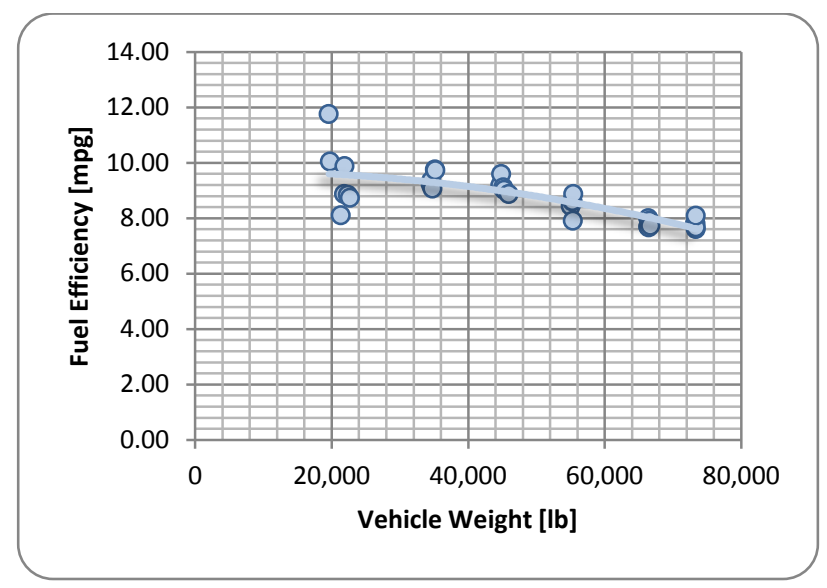

Fig. 31. FE vs. Vehicle Weight, Vehicle Speed between $65 \mathrm{mph}$ to $70 \mathrm{mph}$ (Average: $68.0 \mathrm{mph}$ ).

Flat Terrain.

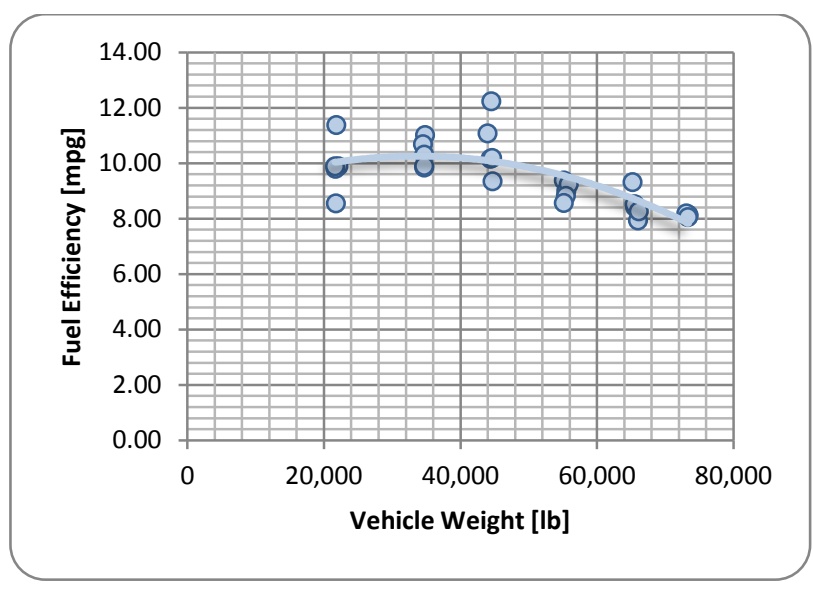

Fig. 30. FE vs. Vehicle Weight, Vehicle Speed between $50 \mathrm{mph}$ to $65 \mathrm{mph}$ (Average: $62.9 \mathrm{mph}$ ). Flat Terrain.

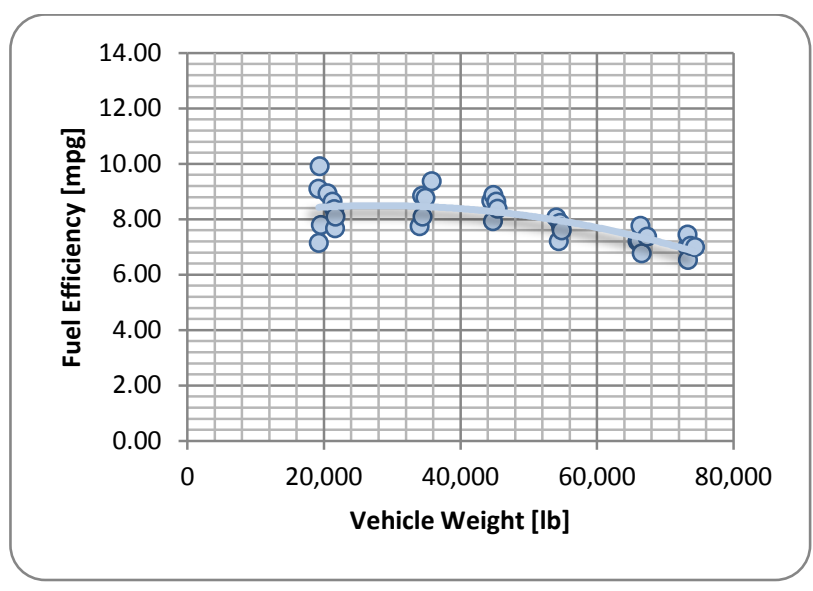

Fig. 32. FE vs. Vehicle Weight, Vehicle Speed between $70 \mathrm{mph}$ to $75 \mathrm{mph}$ (Average: $72.3 \mathrm{mph}$ ). Flat Terrain.

The information shown in Fig. 29 to Fig. 32 was combined into one chart presented in Fig. 33. In that figure, the raw data was fitted with linear regression lines which presented similar coefficients of determination as the ones obtained when the data was fitted with polynomial lines (note: neither regression line types were a good fit for the data). The chart shows that the FE decreases as the vehicle 
weight increases. The figure also shows that, in general, for any given vehicle weight level, the FE decrease as the vehicle speed increases. However, a close examination of the raw data graphed indicates that there are overlaps in FE for any given vehicle weight, at least between adjacent speed ranges. The data presented in chapter two of this report showed that speed variability (a proxy for traffic congestion) had a significant impact on FE. This is most likely the reason of the observed FE variability in Fig. 29 to Fig. 32.

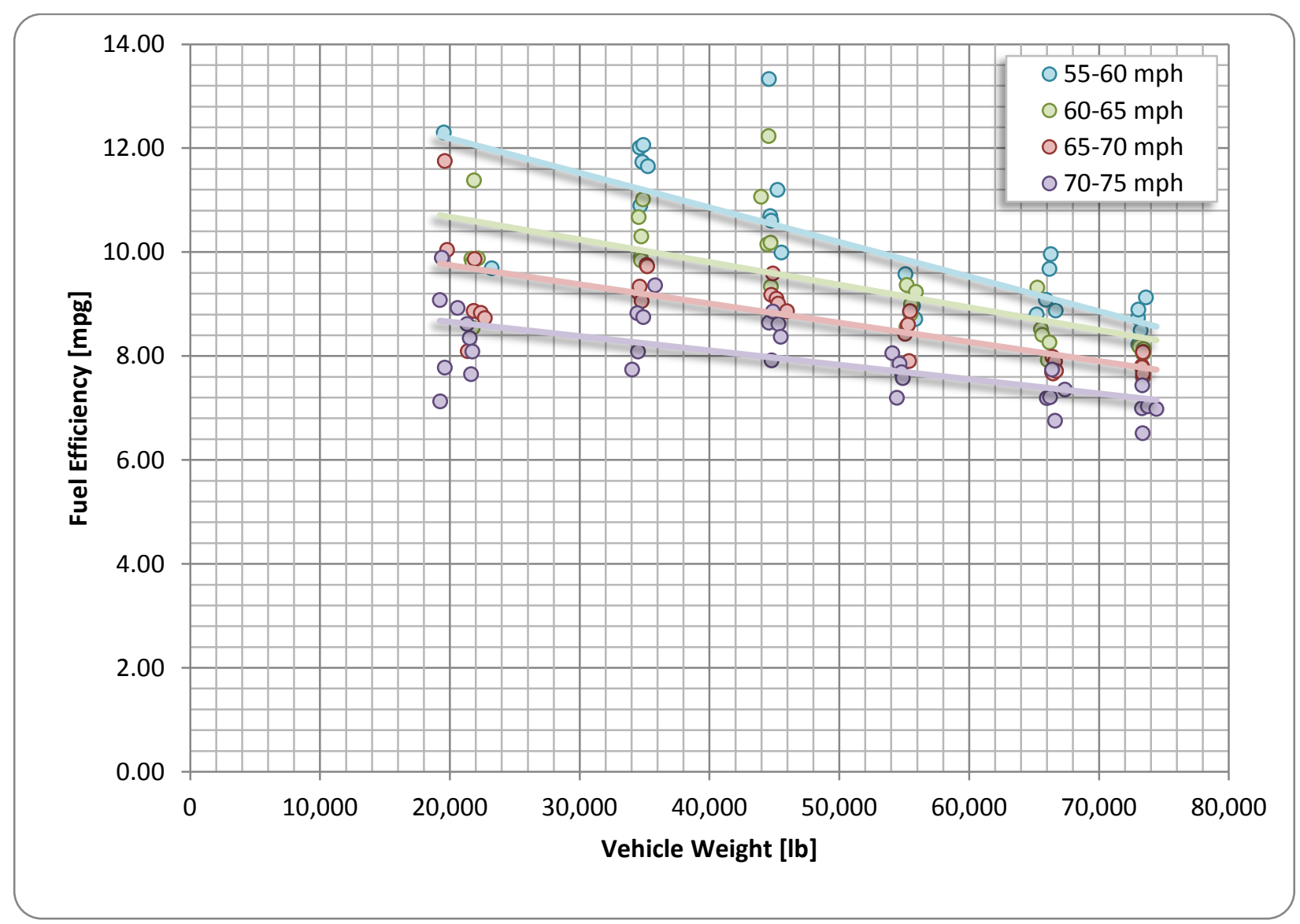

Fig. 33. FE vs. Vehicle Weight for Different Speed Intervals (with Polynomial Regression Lines). Flat Terrain.

\subsubsection{FE Extrapolation as a Function of Vehicle Weight}

The parsed data generated for the analysis discussed in the previous sections could be used to fit a model that predicts FE as a function of weight for a given speed. This model, in turn, can be applied to forecast $\mathrm{FE}$ as a function of vehicle weight outside the data range used to fit it. Of course, as the independent variable (vehicle weight, in this case) moves away from the data range used to build the model, there is less confidence in the forecasted values. Moreover, at some point the forecasting model may breakdown completely if, for example, the input to the model falls outside what the technology (in this case, truck engine) can handle. It may be the case that for vehicle weights higher than a certain threshold, the current Class-8 truck engine models (and in particular the engines with which the data was collected in the HTDC project) may not be able to haul the load, at which point the forecasting model becomes completely 
unreliable. However, for vehicles weights that are reasonably close to the current maximum weights (e.g. $10-20 \%$ ), the prediction model could be used with reasonably confidence.

To build this model, the parsed data was aggregated across vehicle weight levels ranging from 20,000 $\mathrm{lb}$ to $80,000 \mathrm{lb}$ by $10,000 \mathrm{lb}$, and vehicle speeds of around $65 \mathrm{mph}$ were selected (most of the traveled distance in the HTDC database was logged at this speed, which is a common freeway speed limit). For each one of the vehicle weight intervals, the distance traveled at around $65 \mathrm{mph}$ and the fuel consumed were aggregated and used to compute an average $\mathrm{FE}$ for that particular weight range. The information is presented in Table 7, which, besides the distance traveled, fuel consumed, FE and average speed, also includes information about the average vehicle weight for each weight range included in the table.

Table 7. Fuel Efficiency by Vehicle Weight Range Speed: 65.0 mph - Flat Terrain

\begin{tabular}{ccrrrr}
\hline $\begin{array}{c}\text { Weight Range } \\
\text { [lb] }\end{array}$ & $\begin{array}{c}\text { Average } \\
\text { Weight } \\
\text { [lb] }\end{array}$ & $\begin{array}{c}\text { Distance } \\
\text { Traveled } \\
\text { [miles] }\end{array}$ & $\begin{array}{c}\text { Fuel } \\
\text { Consumed } \\
\text { [gal] }\end{array}$ & $\begin{array}{c}\text { Fuel } \\
\text { Efficiency } \\
\text { [mpg] }\end{array}$ & $\begin{array}{c}\text { Average } \\
\text { Speed } \\
\text { [mph] }\end{array}$ \\
\hline $\mathbf{2 0 , 0 0 0 - 3 0 , 0 0 0}$ & 21,222 & 51.4 & 5.4 & 9.5 & 65.0 \\
\hline $\mathbf{3 0 , 0 0 0 - 4 0 , 0 0 0}$ & 34,285 & 505.9 & 53.0 & 9.5 & 65.0 \\
\hline $\mathbf{4 0 , 0 0 0 - 5 0 , 0 0 0}$ & 44,911 & 537.8 & 58.7 & 9.2 & 65.0 \\
\hline $\mathbf{5 0 , 0 0 0 - 6 0 , 0 0 0}$ & 55,468 & 541.2 & 63.3 & 8.6 & 64.9 \\
\hline $\mathbf{6 0 , 0 0 0 - 7 0 , 0 0 0}$ & 66,558 & 1356.9 & 171.9 & 7.9 & 65.0 \\
\hline $\mathbf{7 0 , 0 0 0 - 8 0 , 0 0 0}$ & 73,248 & 1363.1 & 172.3 & 7.9 & 65.0 \\
\hline
\end{tabular}

The average vehicle weight and average FE (columns 2 and 5 in Table 7) were used to build a FE forecasting model presented in Eq. 1 below:

$$
F E(w)=-0.000000000475 w^{2}+0.000008 w+9.6687
$$

where $w$ is the total vehicle weight and $F E(w)$ is the predicted FE for that weight. The coefficient of determination for this model was $\mathrm{R}^{2}=0.953$, a reasonably high value that gives confidence to the fitted model. Fig. 34 shows both the data and the prediction model, together with the forecasting region (shown in red). For vehicle weights of $96,000 \mathrm{lb}$, the model predicts $6.1 \mathrm{mpg}$, or about a $24 \%$ decrease in FE, when compared to the average of the 70,000 lb-80,000 lb range presented in Table 7 . Higher vehicle weights could be used as inputs for the model presented in Eq. 1 above; however, as the vehicle weight increases and it falls further away from the data range used to create the model, the predictions become more unreliable. 


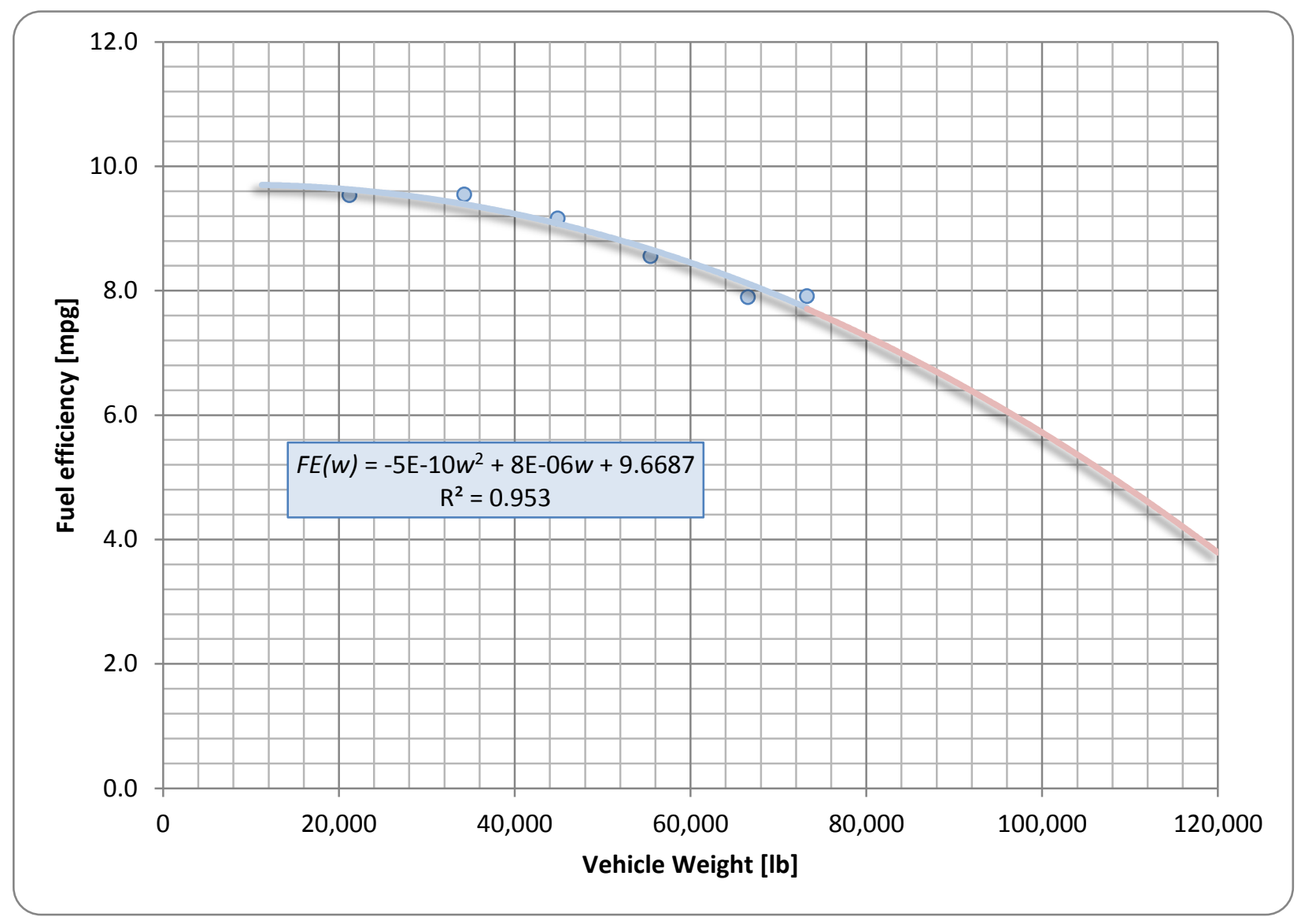

Fig. 34. FE vs. Vehicle Weight. Vehicle Speed: $65 \mathrm{mph}$ - Flat Terrain.

\subsection{EFFECT OF VEHICLE SPEED AND PAYLOAD ON FUEL EFFICIENCY - FLAT TERRAIN}

The information used in the previous two sections to analyze the effects of speed (section 3.1) and vehicle weight (section 3.2) can be combined together to represent FE as a function of these two variables. Fig. 35 shows a three-dimensional representation of the raw data, while Fig. 36 presents the fitted data. The latter clearly shows the combined effect that the vehicle speed and the vehicle weight have on FE while traveling on flat terrain. For light and medium loads, FE decreases considerably as speed increases. In the case of medium-heavy and heavy loads (total vehicle weight larger than 65,000lb), FE tends to increase as the vehicle speed increases from $55 \mathrm{mph}$ (minimum highway speed limit in the US) up to about 58-60 mph. For speeds higher than $60 \mathrm{mph}$, FE decreases at an almost constant rate with increasing speed (see Fig. 36). 


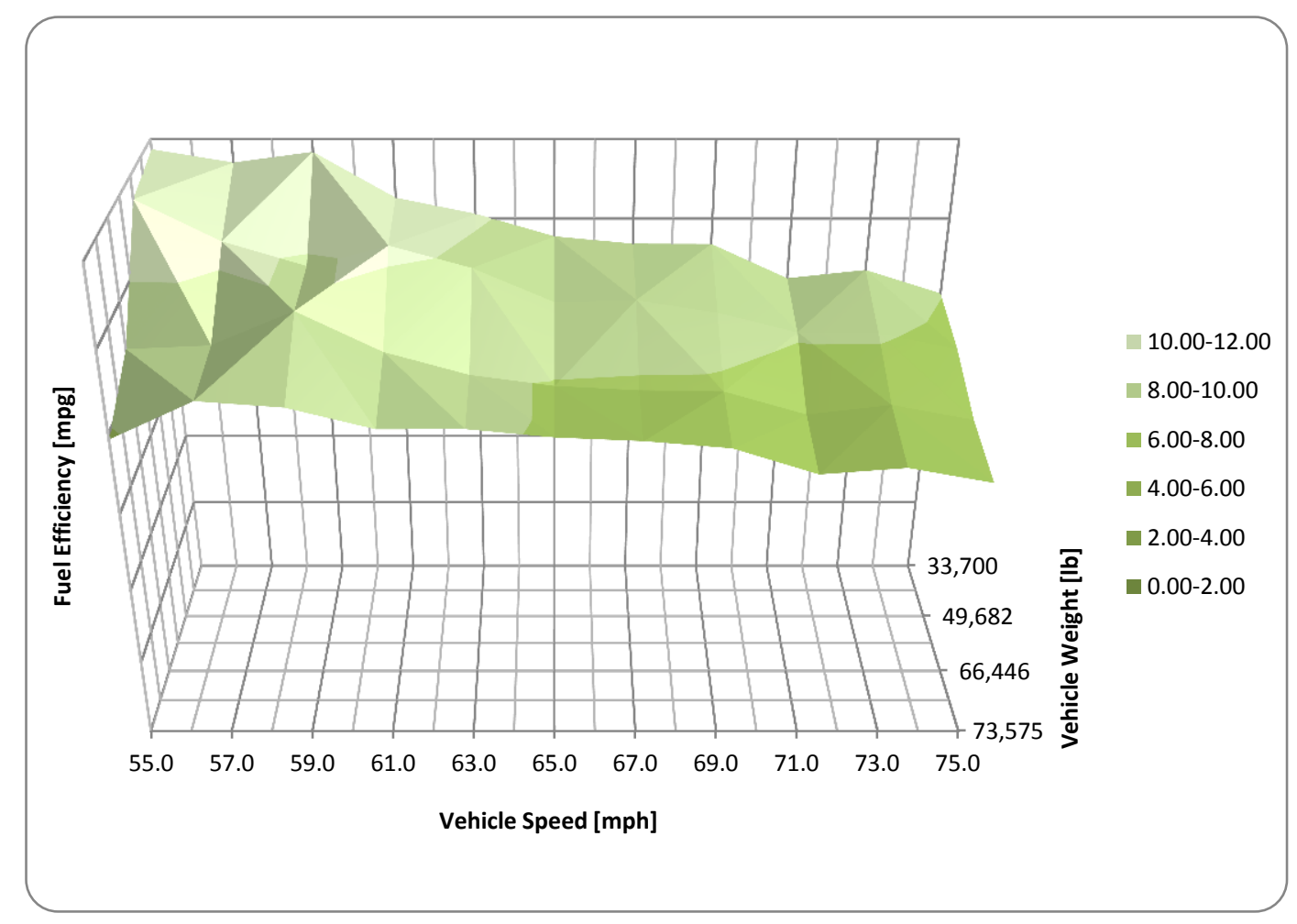

Fig. 35. FE vs. Vehicle Speed and Vehicle Weight (Raw Data) - Flat Terrain.

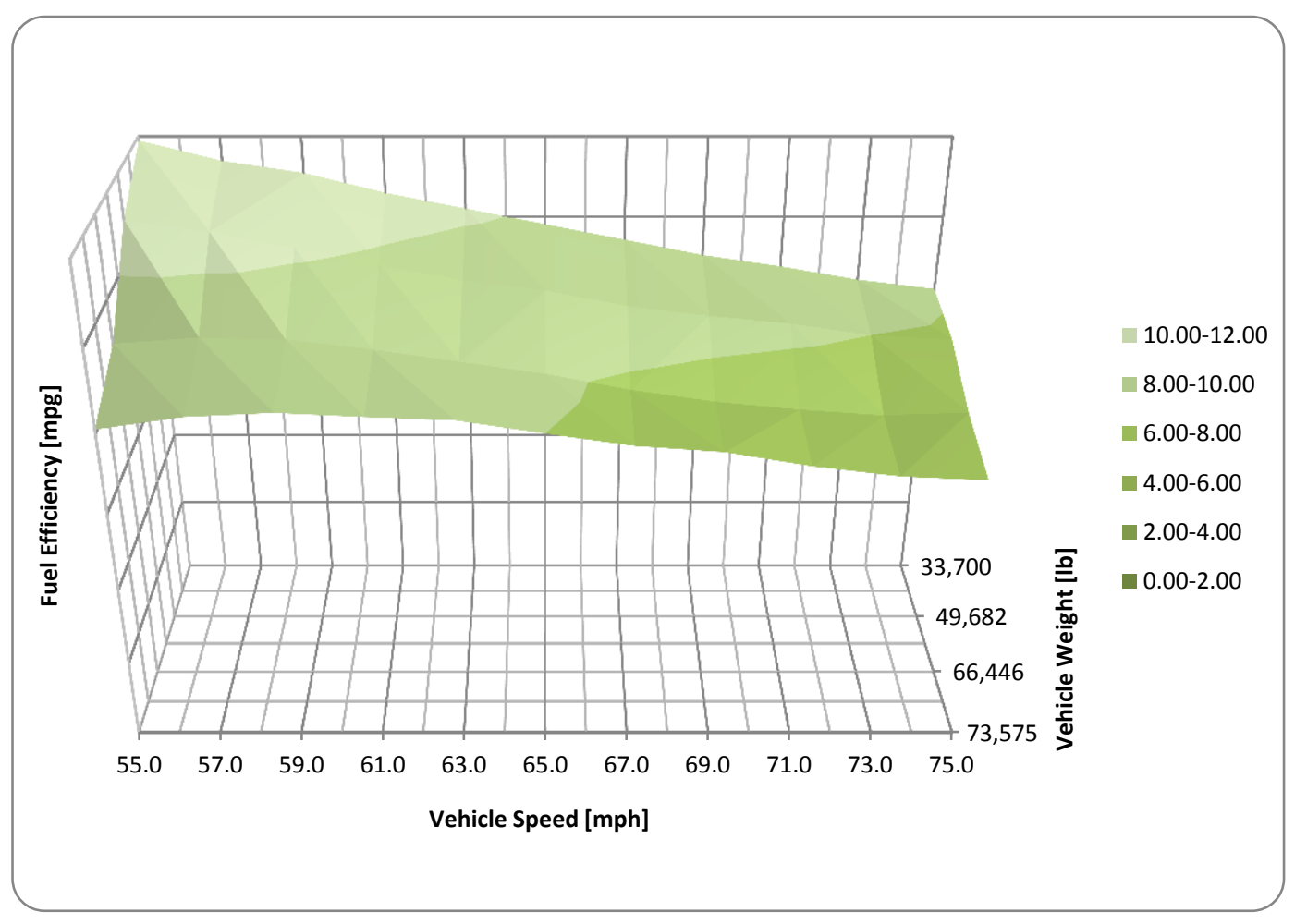

Fig. 36. FE vs. Vehicle Speed and Vehicle Weight (Fitted Data) - Flat Terrain. 


\section{EFFECT OF PAYLOAD AND VEHICLE SPEED ON FUEL EFFICIENCY - UPSLOPE TERRAIN}

This chapter covers the effects that vehicle speed and vehicle weight have on the FE of large trucks while traveling on mild uphill terrains. The methodology to select and parse the data applied here was the same as the one used in the previous chapter, with the exception that the roadway grades considered were between $1 \%$ and $3 \%$ (note: almost $2 / 3$ of the distance traveled on mild uphill terrains was on terrains with $1 \%$ to $2 \%$ grades). This type of terrain covered approximately $20 \%$ of the total distance traveled by the trucks while collecting the HTDC data. The vehicles also logged about $20 \%$ of the total distance while traveling on mild downhill terrain. However, this type of terrain, as well as severe downsloping terrains, which covered almost $4.5 \%$ of the total distance covered in the HTDC project, are irrelevant in terms of the effects of speed and weight on FE since almost no fuel is consumed while a truck is traveling on downhill terrains (unless the vehicle is starting to move from a static position or traveling under congested conditions). Therefore, the cases covered in this and the previous chapters cover almost $100 \%$ of the terrain conditions that are relevant to the FE of large trucks.

\subsection{EFFECT OF VEHICLE SPEED ON FUEL EFFICIENCY - UPSLOPE TERRAIN}

Similarly to the flat terrain case discussed in the previous chapter, the information corresponding to mild upslope terrains was aggregated across all vehicle weights bins to compute distance traveled and fuel consumed at different vehicle speeds. Fig. 37 shows this information for the vehicles in the HTDC database while they were traveling on upslope terrain (1\% to $3 \%$ roadway grade). Notice that as it was the case for flat terrain, the graph has two peaks which, as discussed in the previous chapter, are due to the trucking company implementing a speed governor half way into the study set at $68 \mathrm{mph}$ (i.e., the vehicles could not be accelerated above $68 \mathrm{mph}$ by pressing the accelerator pedal). In this case, however, the second peak is less pronounced than in the flat-terrain case since the vehicles cannot be easily accelerated to high speeds while traveling uphill. Notice also that as opposed to the flat-terrain case, the two curves (distance traveled and fuel consumed) when scaled such that the two maximum coincide, are almost one on top of the other for all the vehicle speeds considered, with very small divergences for speeds between 57 and $67 \mathrm{mph}$. This is an indication that, overall, there is no "preferred" speed at which FE could be optimized while traveling on this type of terrain. 


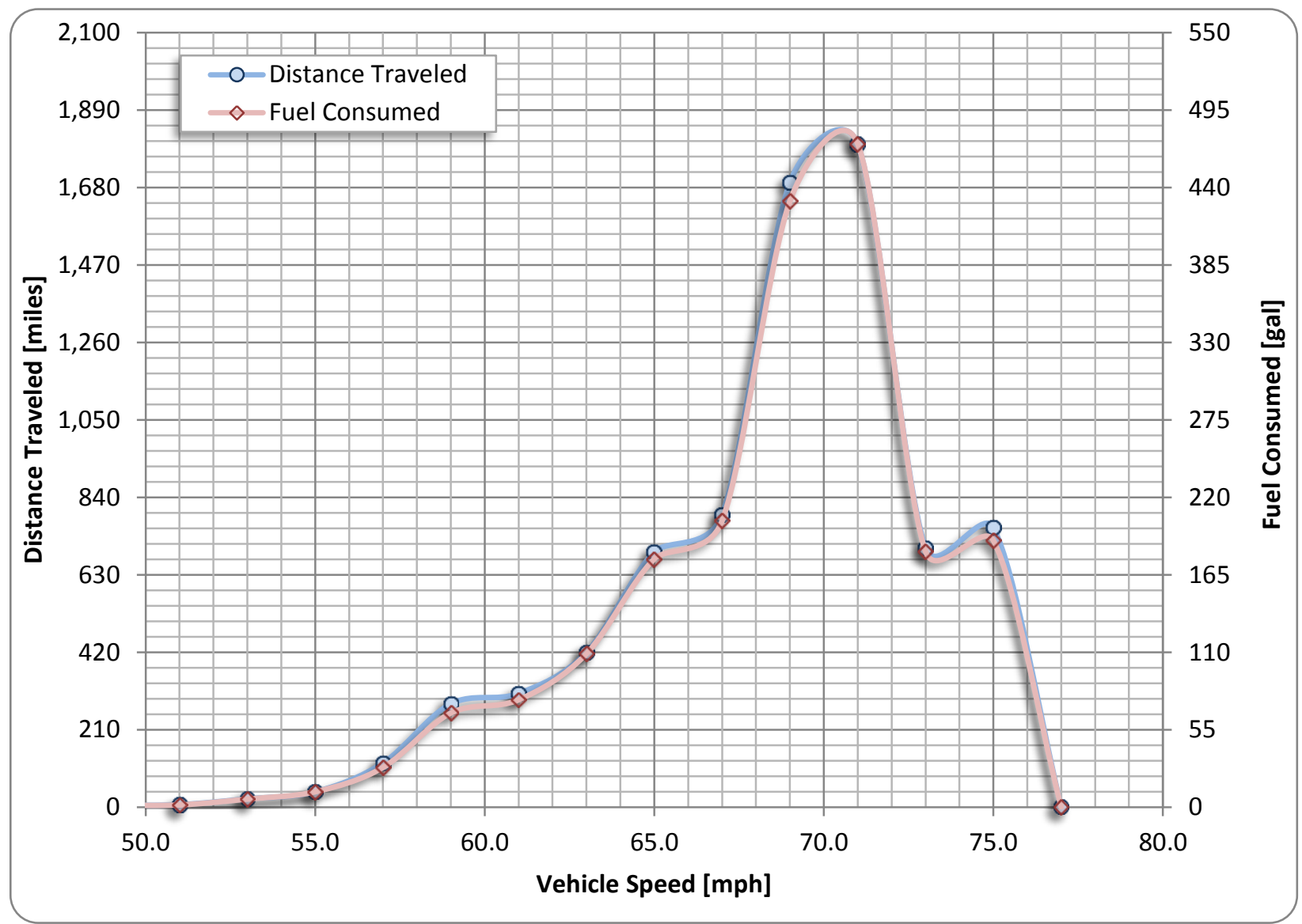

Fig. 37. Total Distance Traveled and Total Fuel Consumed vs. Vehicle Speed. Upslope Terrain.

The information was also aggregated by vehicle weight ranges. Fig. 38 present the relationships between $\mathrm{FE}$ and vehicle speed for vehicles traveling on upslope terrain while hauling loads that resulted in total vehicle weights between $20,000 \mathrm{lb}$ to $40,000 \mathrm{lb}, 40,000 \mathrm{lb}$ to $60,000 \mathrm{lb}, 60,000 \mathrm{lb}$ to 70,000 lb, and 70,000 $\mathrm{lb}$ to $80,000 \mathrm{lb}$. In all of the four cases, the raw data was fitted with a polynomial function of third order (shown in the figure), with coefficients of determination $\mathrm{R}^{2}$ that ranged from 0.29 to 0.95 .

Fig. 38 shows that for light and medium loads (less than 60,000 lbs) the FE decreases almost in a linear fashion as the vehicle speed increases. This is a similar to what was observed while the vehicles were traveling on flat terrain. For heavy loads, FE is almost constant for speeds ranging from 57 to about 66 mph. For speeds higher than $66 \mathrm{mph}$, the FE decreases with speed, but at a lower rate than for light and medium loads. Notice that, as expected, at any given speed, the FE decreases as the vehicle weight increases. This difference appears to be constant as the vehicle speed increases when comparing light vs. medium loads and medium-heavy vs. heavy loads. However, when comparing light vs. heavy loads, the difference decreases as speed increases. 


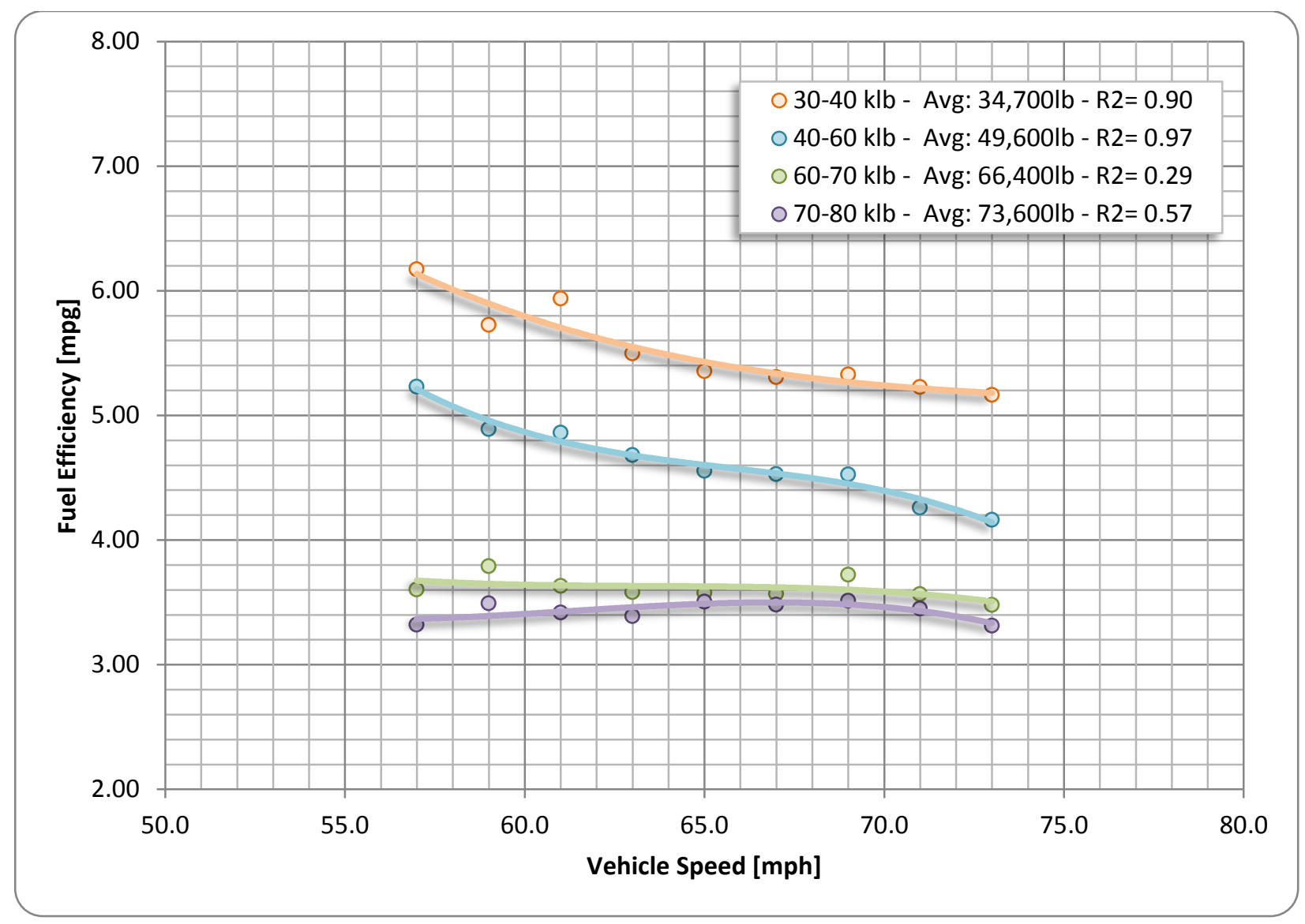

Fig. 38. FE vs. Vehicle Speed for Different Vehicle Weight Levels (Polynomial Regression Lines). Upslope Terrain.

\subsection{EFFECT OF VEHICLE WEIGHT ON FUEL EFFICIENCY - UPSLOPE TERRAIN}

Similarly to the flat terrain case, the processed upslope-terrain data was also aggregated across speed bins to study the effect that the vehicle weight has on FE. The relationships between FE and vehicle weight for speed ranges of 55-60 mph, 60-65 mph, 65-70 mph, and 70-75 mph are presented in Fig. 39. For each speed interval, the figure presents both the raw data and second order polynomial fitting curves, which had coefficients of determination that ranged from 0.87 to 0.99 . Fig. 39 shows that the FE decreases as the vehicle weight increases; with all the curves converging to around $3.4 \mathrm{mpg}$ for very heavy weights, independently of the speed of the vehicle. 


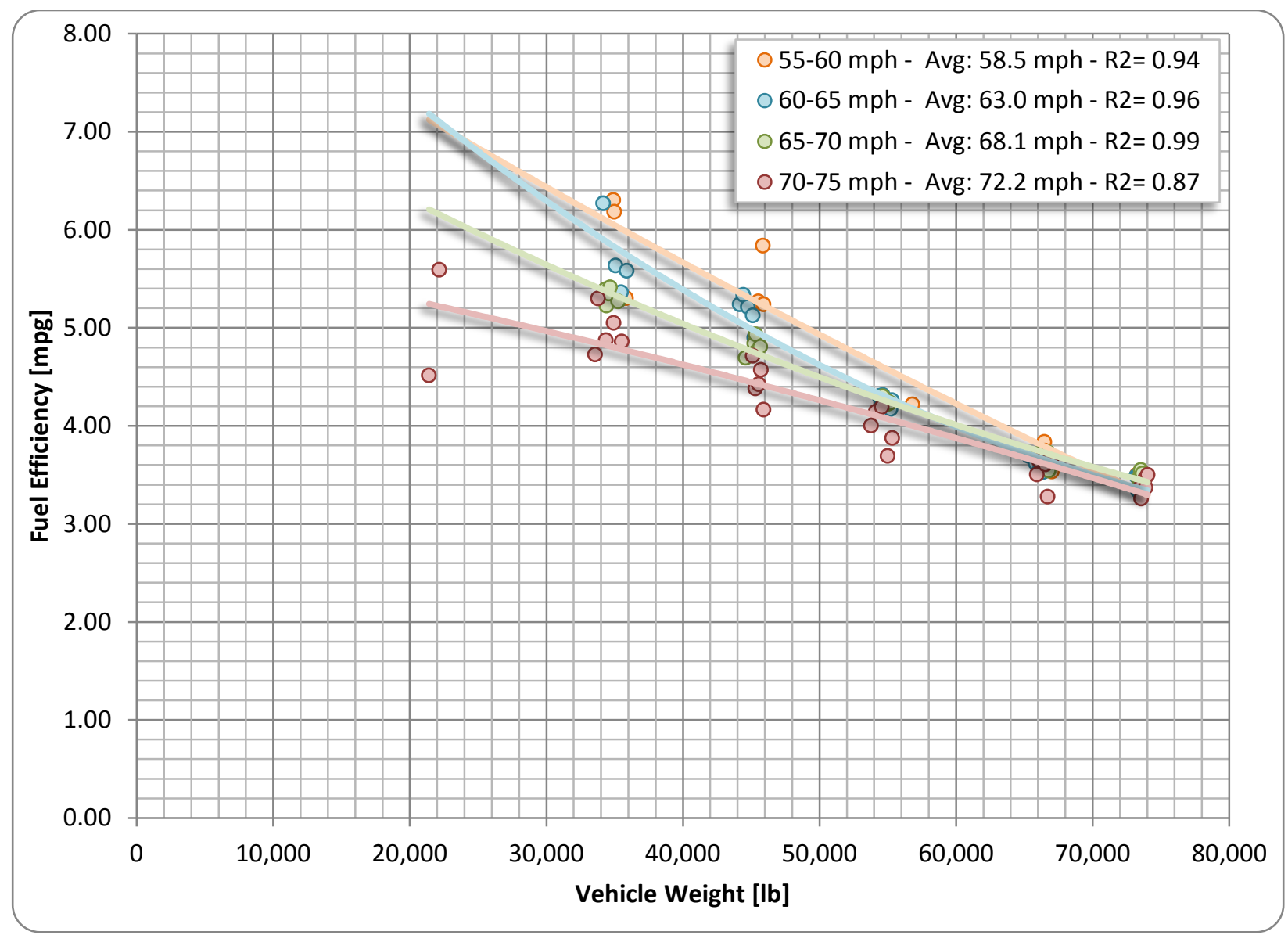

Fig. 39. FE vs. Weight for Different Vehicle Speed Intervals (with Polynomial Regression Lines). Upslope Terrain.

\subsection{EFFECT OF VEHICLE SPEED AND VEHICLE WEIGHT ON FUEL EFFICIENCY - UPSLOPE TERRAIN}

The information used in section 4.1 to analyze the effects of speed on FE and in section 4.2 to study how vehicle weight affects FE was combined to represent FE as a function of these two variables. Fig. 40 and Fig. 41 display this information for the raw data and fitted data, respectively. The latter clearly shows that for uphill terrains with grades between $1 \%$ and $3 \%$, the FE of Class- 8 trucks decreases abruptly with vehicle weight ranging from light loads up to medium-heavy loads (total vehicle weight larger than $65,000 \mathrm{lb}$ ). After that, increases in the vehicle weight only decreases FE slightly. FE also decreases significantly with speed, but only for light and medium loads. For medium-heavy and heavy loads, speed has practically no impact on FE for vehicles traveling on uphill terrains. 


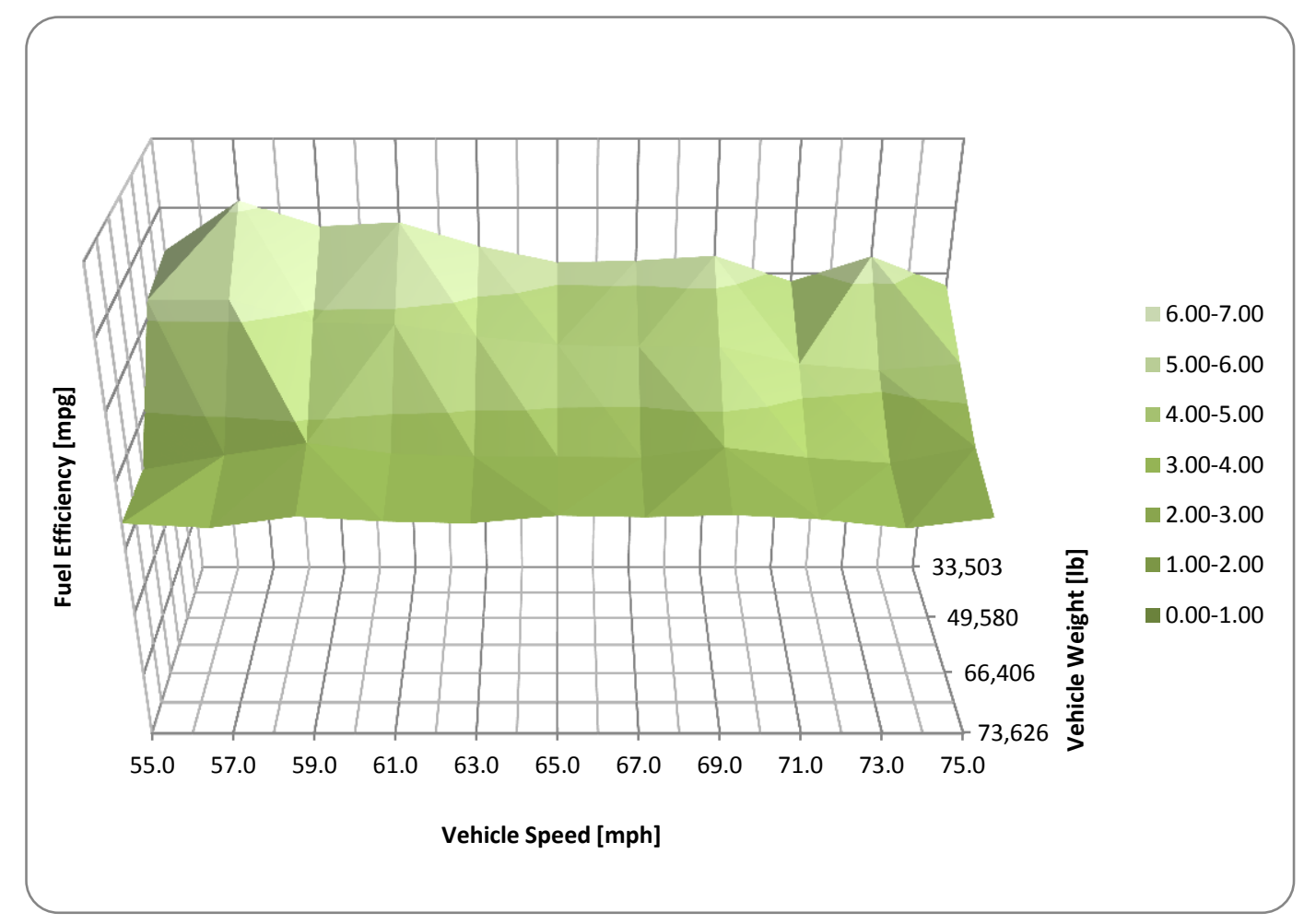

Fig. 40. FE vs. Vehicle Speed and Vehicle Weight (Raw Data) - Upslope Terrain.

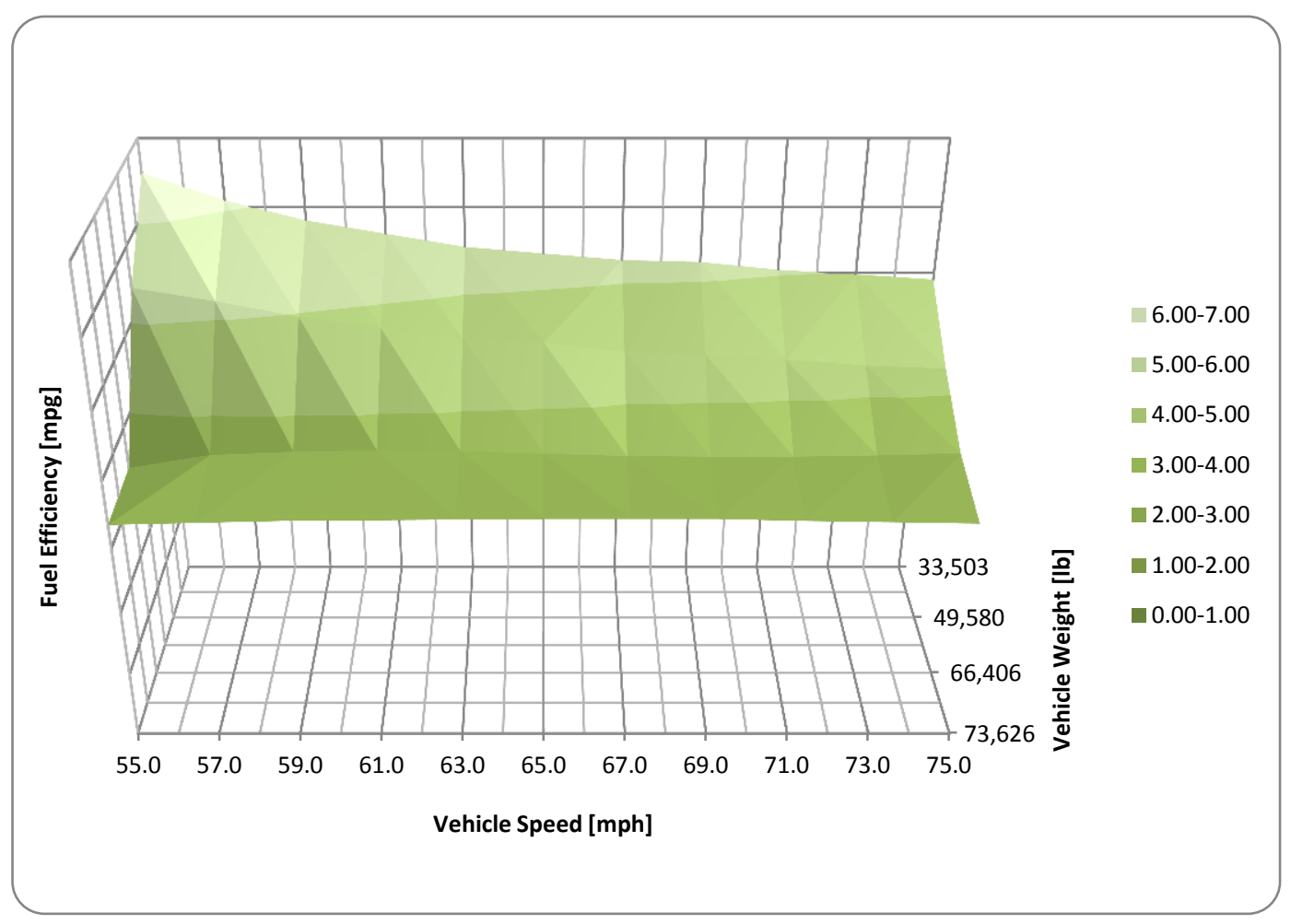

Fig. 41. FE vs. Vehicle Speed and Vehicle Weight (Fitted Data) - Upslope Terrain. 


\section{COMPARISSON OF FUEL EFFICIENCIES AT DIFFERENT SPEEDS - FLAT TERRAIN}

Chapter 2 of this report showed that for medium-heavy and heavy loads (i.e., total vehicle weight larger than $65,000 \mathrm{lb}$ ), the FE of Class-8 trucks presented a maximum (i.e., optimal operation in terms of fuel consumption) when the vehicle was traveling at speeds ranging from 58 to $60 \mathrm{mph}$ on flat terrain (-1\% to $1 \%$ roadway grade). This is clearly depicted in Fig. 42.

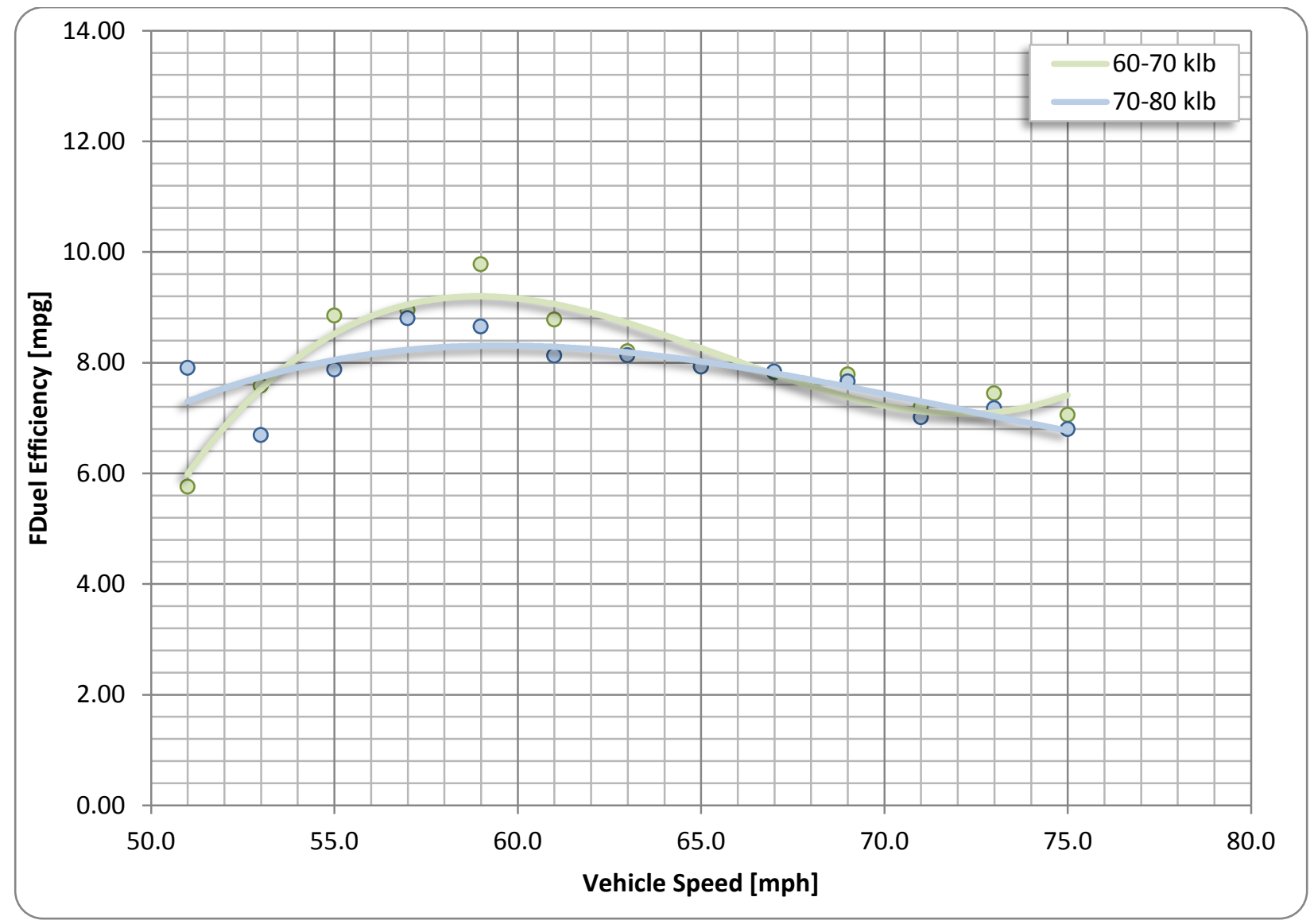

Fig. 42. FE vs. Vehicle Speed for Two Vehicle Weight Levels - Flat Terrain.

In this chapter, statistical analyses are conducted to determine if there are any statistically significant differences in FEs of heavy trucks hauling medium to heavy loads when traveling at different speed on flat terrains. Four speeds where selected, three of them (i.e., $55 \mathrm{mph}, 65 \mathrm{mph}$, and $70 \mathrm{mph}$ ) correspond to the most common freeway speed limits in the nation. The fourth selected speed was $59 \mathrm{mph}$ and is the one at which the FE of Class-8 trucks traveling under the terrain and load conditions described here appears to be optimal (i.e., maximum distance traveled per gallon of fuel). 


\subsection{DATA CHARACTERIZATION}

The parsed data described in the second chapter of this report was divided into ten-mile segments. The FE achieved was computed for each one of segments, and it became one observation for the statistical analyses conducted in this section. Each observation was also associated to a given vehicle speed and vehicle weight. The entire database of flat terrain ten-mile segments was then sorted by vehicle speed and observations that fell within a two-mph interval around the four analyzed speeds (i.e., $55 \mathrm{mph}, 59 \mathrm{mph}, 65$ $\mathrm{mph}$, and $70 \mathrm{mph}$ ) were selected for further consideration. Within these two-mph intervals, the observations were sorted by vehicle weight and the average speed and average weight were computed. If the average speed was not exactly the speed that was selected for the analysis, some observations at both ends of the two-mph interval were discarded until the desired average speed was reached. The same procedure was used to reach similar average vehicle weights for the four considered speed datasets. The average FE and the standard deviation of the FE within each of the four datasets were then computed.

Table 8 presents the information that was used for the statistical comparison of the effect that speed has on FE of Class- 8 trucks. The table presents the number of observations in each of the four datasets, the average speed for that dataset, average weight, average roadway grade, average FE, and the standard deviation of the distribution of the FE values within each dataset. Notice that, as expected, the $59 \mathrm{mph}$ speed dataset has the highest average FE of all the selected speeds.

Fig. 43 to Fig. 46 present, in graphical form, the distributions of vehicle speed, vehicle weight, roadway grade, and FE for each one of the four selected datasets, respectively. Because the different datasets had very different number of observations, the frequency (ordinate axis) of the distributions is presented as percentage of total observations in the dataset for each one of the selected analysis speeds rather than as the absolute number of observations. The figures show that the distributions of weight and roadway grade have similar composition for the four selected speed. The distribution of FE, on the other hand, shows that the $59 \mathrm{mph}$ and $55 \mathrm{mph}$ speed datasets tended to have higher FEs than the other two speeds.

Table 8. Fuel Efficiency by Speed for Flat Terrain and Vehicle Weight 70,000 lb to 80,000 lb

\begin{tabular}{rrrrrr}
\hline $\begin{array}{c}\text { Sample } \\
\text { Size }\end{array}$ & $\begin{array}{c}\text { Average } \\
\text { Speed } \\
\text { [mph] }\end{array}$ & $\begin{array}{c}\text { Average } \\
\text { Weight } \\
\text { [lb] }\end{array}$ & $\begin{array}{c}\text { Average } \\
\text { Grade } \\
{[\%]}\end{array}$ & $\begin{array}{c}\text { Average } \\
\text { FE } \\
\text { [mpg] }\end{array}$ & $\begin{array}{c}\text { Std. Dev. of } \\
\text { FE } \\
\text { [mpg] }\end{array}$ \\
\hline 20 & 55.0 & 60,367 & $0.03 \%$ & 9.75 & 2.81 \\
122 & 59.0 & 60,298 & $0.01 \%$ & 10.02 & 2.13 \\
397 & 65.0 & 60,302 & $0.02 \%$ & 8.58 & 1.59 \\
1045 & 70.0 & 60,312 & $0.01 \%$ & 7.98 & 1.32 \\
\hline
\end{tabular}




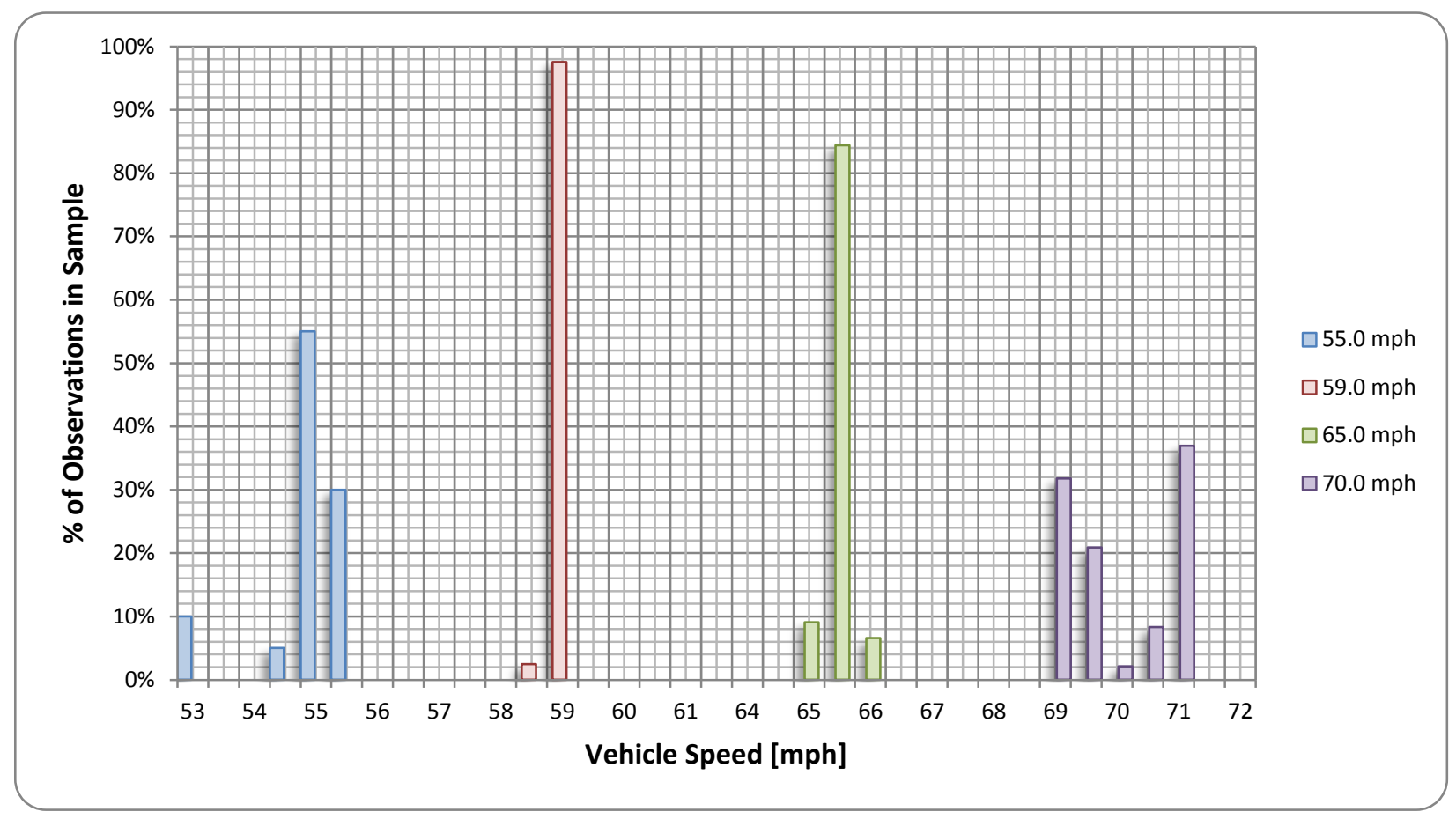

Fig. 43. Dataset Distributions of Vehicle Speed for Four Analysis Speeds. Flat Terrain.

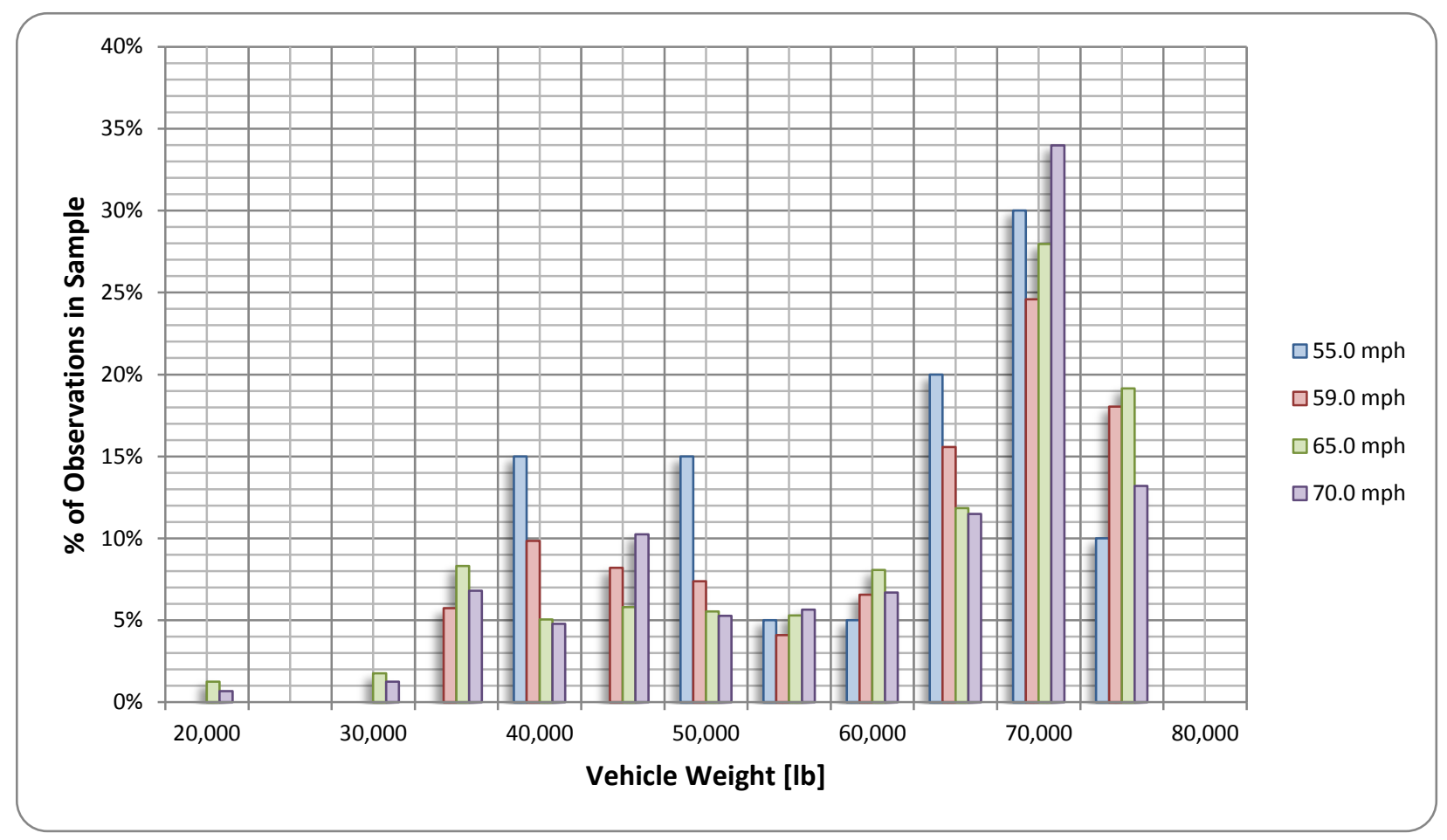

Fig. 44. Dataset Distributions of Vehicle Weight for Four Analysis Speeds. Flat Terrain. 


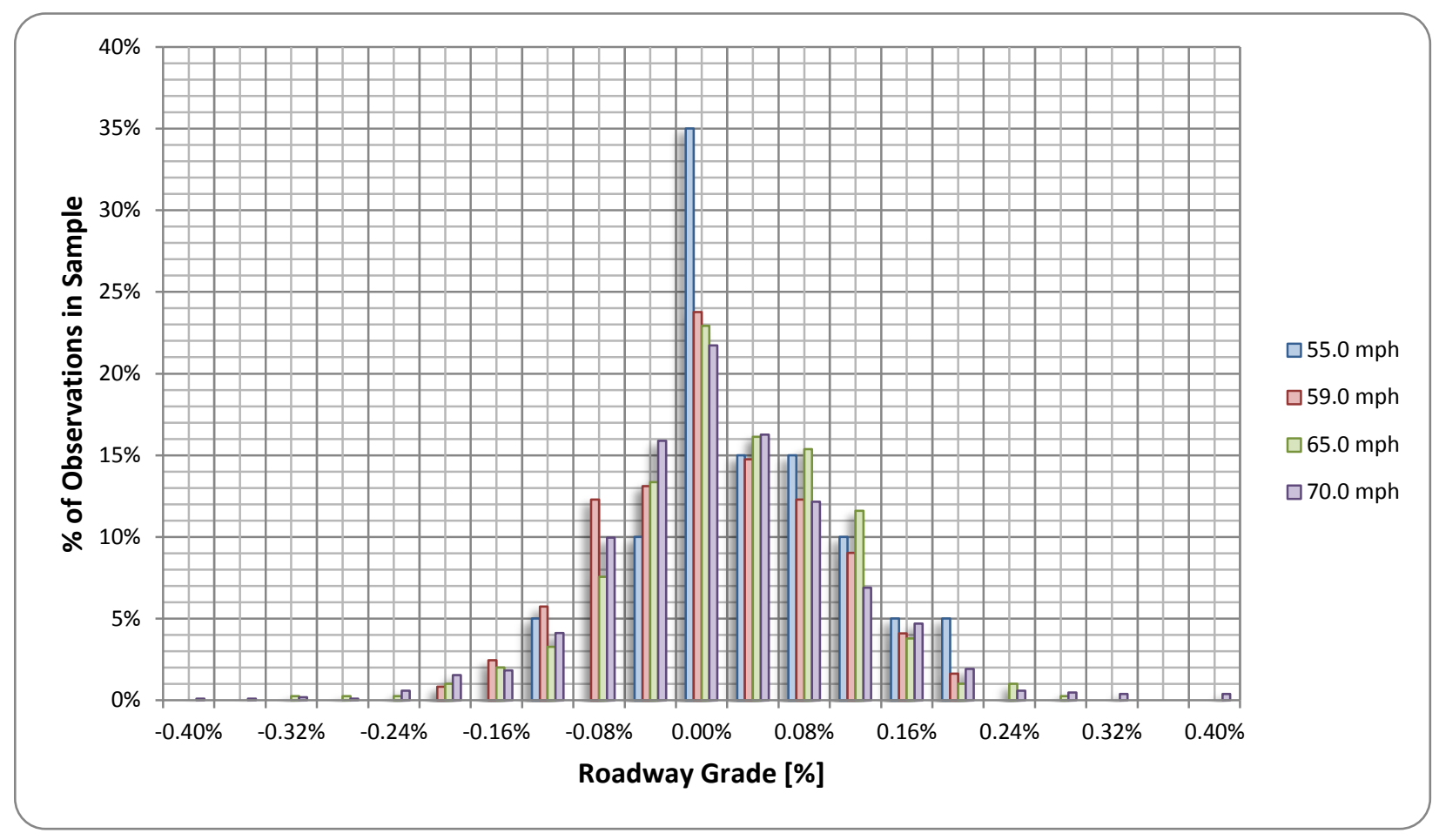

Fig. 45. Dataset Distributions of Roadway Grades for Four Analysis Speeds. Flat Terrain.

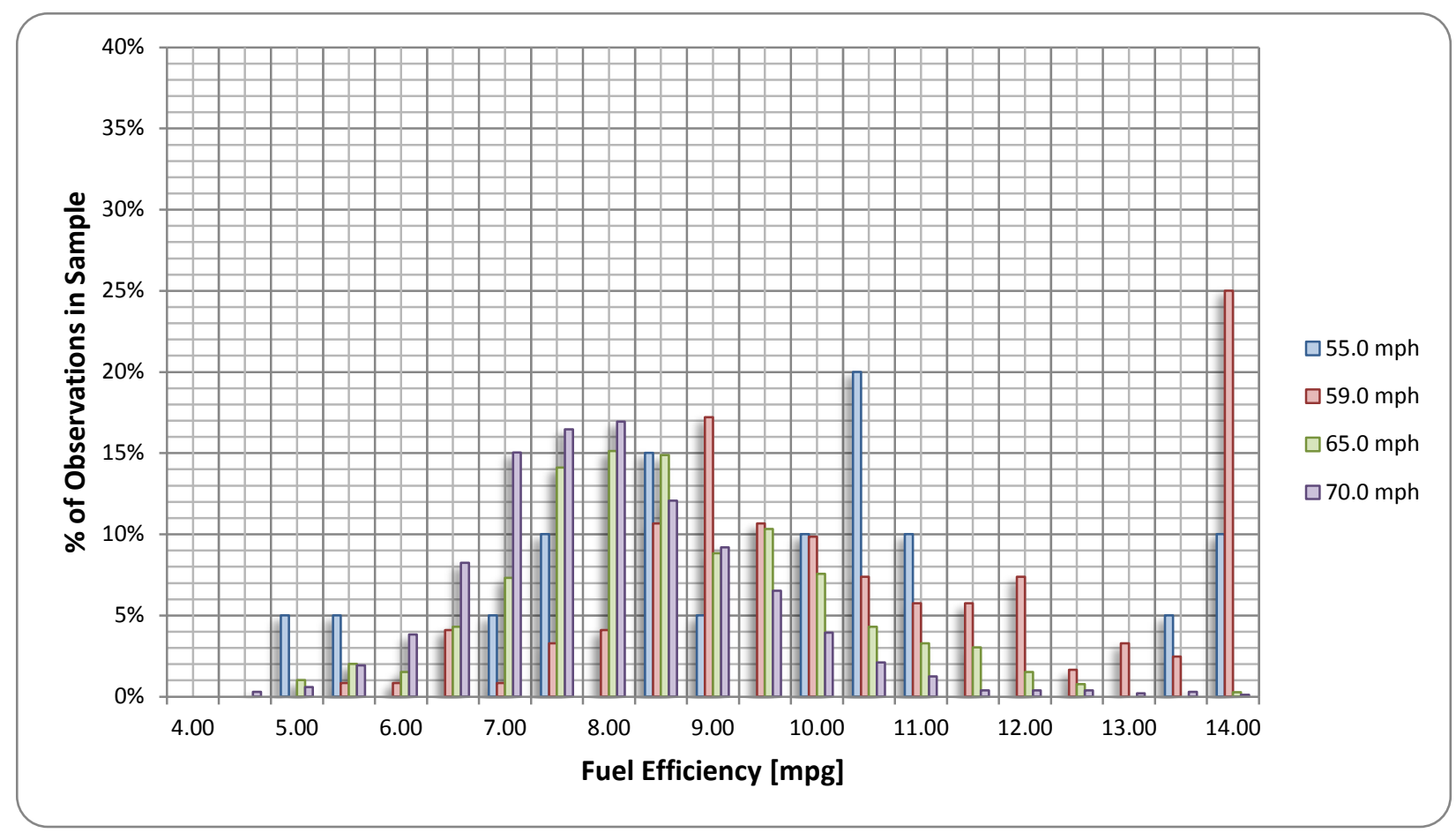

Fig. 46. Dataset Distributions of Vehicle Fuel Efficiency for Four Analysis Speeds. Flat Terrain. 


\subsubsection{Statistical Analysis}

To statistically corroborate that vehicles traveling at $59 \mathrm{mph}$ have higher FEs for the weight and terrain conditions described above, a test of hypothesis was performed [6]. That is, a null hypothesis asserting that the there is no difference between the distribution of fuel efficiencies for any of the three highway speed limits (55 mph, $65 \mathrm{mph}$, and $70 \mathrm{mph}$ ) and that of the base case (i.e., $59 \mathrm{mph}$ ) was tested against an alternative hypothesis stating that the average of the distributions of the fuel efficiencies of the former are smaller than that of the latter. In other words, calling $\mu_{x x}$ the mean of the distribution of 10-mile segment fuel efficiencies corresponding to the freeway speed limits, and $\mu_{59}$ the mean of the distribution of 10-mile segment fuel efficiencies computed for vehicles traveling at $59 \mathrm{mph}$; then the null and alternative hypotheses are represented by equations (2) and (3) below.

$$
\begin{aligned}
& \mathrm{H}_{\mathrm{o}}: \mu_{x x}=\mu_{59} \\
& \mathrm{H}_{\mathrm{a}}: \mu_{x x}<\mu_{59} \quad \text { (Eq. 2) }
\end{aligned}
$$

Table 9 presents a summary of the statistics describing each one of the four FE distributions. For each one of the analyzed speeds, the first three rows of the table show the average (same as the ones presented in Table 8), standard deviation and sample size of each of the distributions. Notice that the minimum sample size is 20 observations ( $55 \mathrm{mph}$ ) with a maximum of 1,045 observations (70 $\mathrm{mph}$ ). The fourth row in Table 9 presents the mean of the difference between the sample average FE of the speed of the corresponding column and that of the $59 \mathrm{mph}$ case. The fifth row shows the standard deviation of the difference which, together with the mean and sample size, permits the computation of the test statistic value $\mathrm{z}$ (a one-tailed test based on the alternative hypothesis shown in Eq. 3 and provided in row six of Table 9). For the $65 \mathrm{mph}$ and $70 \mathrm{mph}$ speeds, the null hypothesis, which states that there is no difference in terms of FE that can be explained by the vehicle traveling at these speeds or at $59 \mathrm{mph}$, can be rejected with more than $99.9 \%$ confidence in favor of the alternative hypothesis, which indicates that, on average, the fuel efficiencies obtained when the vehicle is traveling at $59 \mathrm{mph}$ is higher than when it is traveling at $65 \mathrm{mph}$ or $70 \mathrm{mph}$ (note: the Type II error -i.e., the probability of failing to reject the null hypothesis when the alternative hypothesis is true - was $18 \%$ and $6 \%$, respectively). When comparing the FE corresponding to the vehicle traveling at $59 \mathrm{mph}$ against the vehicle traveling at $55 \mathrm{mph}$, the test fails to reject the null hypothesis, thus suggesting that there is no difference in the average FEs obtained at these two speeds (note: the main reason for this result is the low number of observations that the $55 \mathrm{mph}$ case has). The Type II error in this case was $46 \%$.

Table 9. Comparison of Fuel Efficiencies for Different Speed Levels Average Vehicle Weight 60,300lb - Average Roadway Grade 0.01\%

\begin{tabular}{lrrrr}
\hline \multirow{2}{*}{ FE Statistics } & \multicolumn{4}{c}{ Speed } \\
\cline { 2 - 5 } & $\mathbf{5 9 . 0} \mathbf{~} \mathbf{m h}$ & $\mathbf{5 5 . 0} \mathbf{~} \mathbf{p h}$ & $\mathbf{6 5 . 0} \mathbf{~} \mathbf{p h}$ & $\mathbf{7 0 . 0} \mathbf{~} \mathbf{p h}$ \\
\hline Mean & 10.02 & 9.75 & 8.58 & 7.98 \\
\hline Std Dev & 2.13 & 2.81 & 1.59 & 1.32 \\
\hline $\mathbf{N}$ & 122 & 20 & 397 & 1,045 \\
\hline Delta Mean & & -0.2751 & -1.4415 & -2.0431 \\
\hline Std Dev Diff & & 0.6569 & 0.2091 & 0.1975 \\
$\mathbf{Z}$ & & 0.4187 & 6.8928 & 10.3425 \\
\hline P-value & & 0.34 & 0.00 & 0.00 \\
Reject $\mathbf{H}_{\mathbf{o}}$ at & & Fails to Reject & $>99.9 \%$ & $>99.9 \%$ \\
confidence level $=$ & & & & \\
\hline
\end{tabular}




\subsection{CONCLUSIONS}

It was observed in the second chapter of this report that vehicles hauling medium-heavy and heavy loads have higher FEs when traveling at speeds between $58 \mathrm{mph}$ and $60 \mathrm{mph}$. The HTDC database was parsed to generate ten-mile freeway segments of flat-terrain, whit the vehicles hauling about $60,300 \mathrm{lb}$ and traveling at highway speed limits of $55 \mathrm{mph}, 65 \mathrm{mph}$, and $70 \mathrm{mph}$, as well as the identified optimal speed of $59 \mathrm{mph}$. Statistical tests were conducted to compare the mean of the distributions of the FEs corresponding to each one of the highway speed limits against the average FE of the $59 \mathrm{mph}$ speed. The statistical tests indicated that it was possible to reject the null hypothesis that speeds of $65 \mathrm{mph}$ and 70 mph did not make any difference in terms of average FEs when compared to a speed of $59 \mathrm{mph}$ in favor of the alternative hypothesis stating that FEs were, on average, higher when the vehicle traveled at 59 $\mathrm{mph}$ that when it did at $65 \mathrm{mph}$ or at $70 \mathrm{mph}$. When the 59-mph FEs were compared against those obtained when the vehicle traveled at $55 \mathrm{mph}$, the null hypothesis could not be rejected, thus indicating that there was not a statistically significant difference between the averages of FE distributions obtained at these two speeds. In this case, although the average FE for the $59 \mathrm{mph}$ was larger than that of the 55 mph case, the number of observations for latter was very small, which diminished the power of the statistical test. 


\section{SUMMARY AND CONCLUSIONS}

The objective of the present study was to investigate the effects that vehicle weight, roadway grade (terrain profile), and speed have on the fuel efficiency (FE) of Class-8 freight trucks. The research was conducted using the extensive database of information collected in the DOE HTDC project (conducted by ORNL in 2006-08), and it was divided into three sub-studies.

In the first one, trips that traversed the same segment of freeway and for which the weight of the tractor and trailer was known were extracted from the database, and fuel efficiencies and other statistics were computed for each trip. Three distinct segments were considered for the analysis, all of which started in Knoxville, TN. One of them contained four forty-mile sub-segments (trips to Nashville, TN on I-40 westbound), while the other were divided into two forty-mile sub-segments (trips to Cleveland, TN on I75 southbound and trips to London, KY on I-75 northbound). For each segment, terrain type and vehicle speed were aggregated over the length of each 40-mile sub-segments. A total of 140 trips were analyzed and three type of terrains were considered: flat terrain (62 trips); flat-upslope-downslope terrain (28 trips); and rolling-upslope-downslope terrain (50 trips). In general, and as expected, FE decreased as the total vehicle weight increased. The terrain also had a very important effect, with uphill terrains severely decreasing FEs. Regarding speed, FE increased as speed increased up to a certain speed and then it started to decrease again. The speed variability (speed standard deviation divided by the average speed in any given sub-segment) which is a proxy for congestion, had a negative effect on FE.

In the second sub-study, the HTDC data was parsed by roadway grade categories, vehicle speed intervals (of 1 and $2 \mathrm{mph}$ ), and vehicle weight levels going from tractor only to fully loaded vehicles by 5,000 lb. For two of the terrain categories (i.e., flat terrain and mild upslope terrain) which cover over $70 \%$ of the total miles logged in the HTDC project, the effect of vehicle weight and vehicle speed on FE was investigated using the parsed data. In the flat-terrain case, the results of the study showed that for light and medium loads, FE decreases considerably as speed increases. In the case of medium-heavy and heavy loads (total vehicle weight larger than 65,000 lb), FE tends to increase as the vehicle speed increases from $55 \mathrm{mph}$ (minimum highway speed limit in the U.S.) up to about 58-60 mph. For speeds higher than $60 \mathrm{mph}$, FE decreases at an almost constant rate with increasing speed. At any given speed, FE decreases and vehicle weight increases, although the relationship between FE and vehicle weight is not linear, especially for vehicle above $65,000 \mathrm{lb}$. A FE prediction model as a function of the vehicle weight was also developed using the flat-terrain data, for vehicle speeds of $65 \mathrm{mph}$. The model predicts a decrease of about $24 \%$ in FE when the vehicle weight is increase from the current limit to 96,000 lb.

The analysis of the information collected while the vehicles were traveling on mild upslope terrains showed that the FE of Class- 8 trucks decreases abruptly with vehicle weight ranging from light loads up to medium-heavy loads. After that, increases in the vehicle weight only decrease FE slightly. FE also decreases significantly with speed, but only for light and medium loads. For medium-heavy and heavy, FE is almost constant for speeds ranging from 57 to about $66 \mathrm{mph}$. For speeds higher than $66 \mathrm{mph}$, the FE decreases with speed, but at a lower rate than for light and medium loads.

Some of the findings of the second sub-study showed that for medium-heavy to heavy loads (i.e., total vehicle weight larger than $65,000 \mathrm{lb}$ ), FE increased with speed up to about $59 \mathrm{mph}$ and then started to decrease for higher speeds. Statistical analyses were conducted to determine the level of significance of this effect. The analyses compared the FEs obtained when the vehicles were traveling at $59 \mathrm{mph}$ vs. the FEs obtained when traveling at highway speed limits (i.e., $55 \mathrm{mph}, 65 \mathrm{mph}$, and $70 \mathrm{mph}$ ). The statistical tests indicated that the FEs were on average higher when the vehicle traveled at $59 \mathrm{mph}$ that when it did at 
$65 \mathrm{mph}$ or at $70 \mathrm{mph}$ (these results were statistically significant at the $99.9 \%$ confidence level). When the 59-mph FEs were compared against the 55-mph FEs, and although the average FE for the former was higher than that of the latter, the low number of $55 \mathrm{mph}$ observations diminished the power of the statistical test and therefore no statistically significant conclusions could be derived from the test. 


\section{REFERENCES}

1. Bohman, M. On Predicting Fuel Consumption and Productivity of Wheel Loaders. Master's Thesis, Department of Mathematics, Luleå University of Technology, Porsön, Luleå, Sweden, 2006. http://epubl.luth.se/1402-1617/2006/009/LTU-EX-06009-SE.pdf. Accessed: June 20, 2011.

2. Hogan, J.; D. Watson, and T. Harrison. Data Points and Duration for Estimating Fuel Consumption of a LPG Engine. In Agricultural Engineering International: the CIGR eJournal, Manuscript PM 07 017. Vol. IX. November, 2007.

3. On-Road Emissions Testing of 18 Tier 1 Passenger Cars and 17 Diesel Powered Public Transport Busses. Final Report, EPA, Reference\# QT-MI-01-000659, 2002. http://www.epa.gov/otaq/models/ngm/r02030.pdf. Accessed June 20, 2011.

4. Browand, F., C. Radovich, and M. Boivin. Fuel Savings by Means of Flaps Attached to the Base of a Trailer: Field Test Results. Transactions Journal of Passenger Cars: Mechanical Systems, SAE 2005 World Congress \& Exhibition, Detroit, MI April 2005

5. Capps, G; O Franzese, B Knee, M B Lascurain, and P Otaduy. Class- 8 Heavy Truck Duty Cycle Project Final Report, ORNL/TM-2008/122, 2008.

6. Devore, J., Probability and Statistics for Engineering and the Sciences, Second Edition, 1987. 



\section{APPENDIX A.}

HTDC DATABASE GEOGRAPHICAL COVERAGE AND GENERAL STATISTICS 



\section{APPENDIX A. HTDC DATABASE GEOGRAPHICAL COVERAGE AND GENERAL STATISTICS}

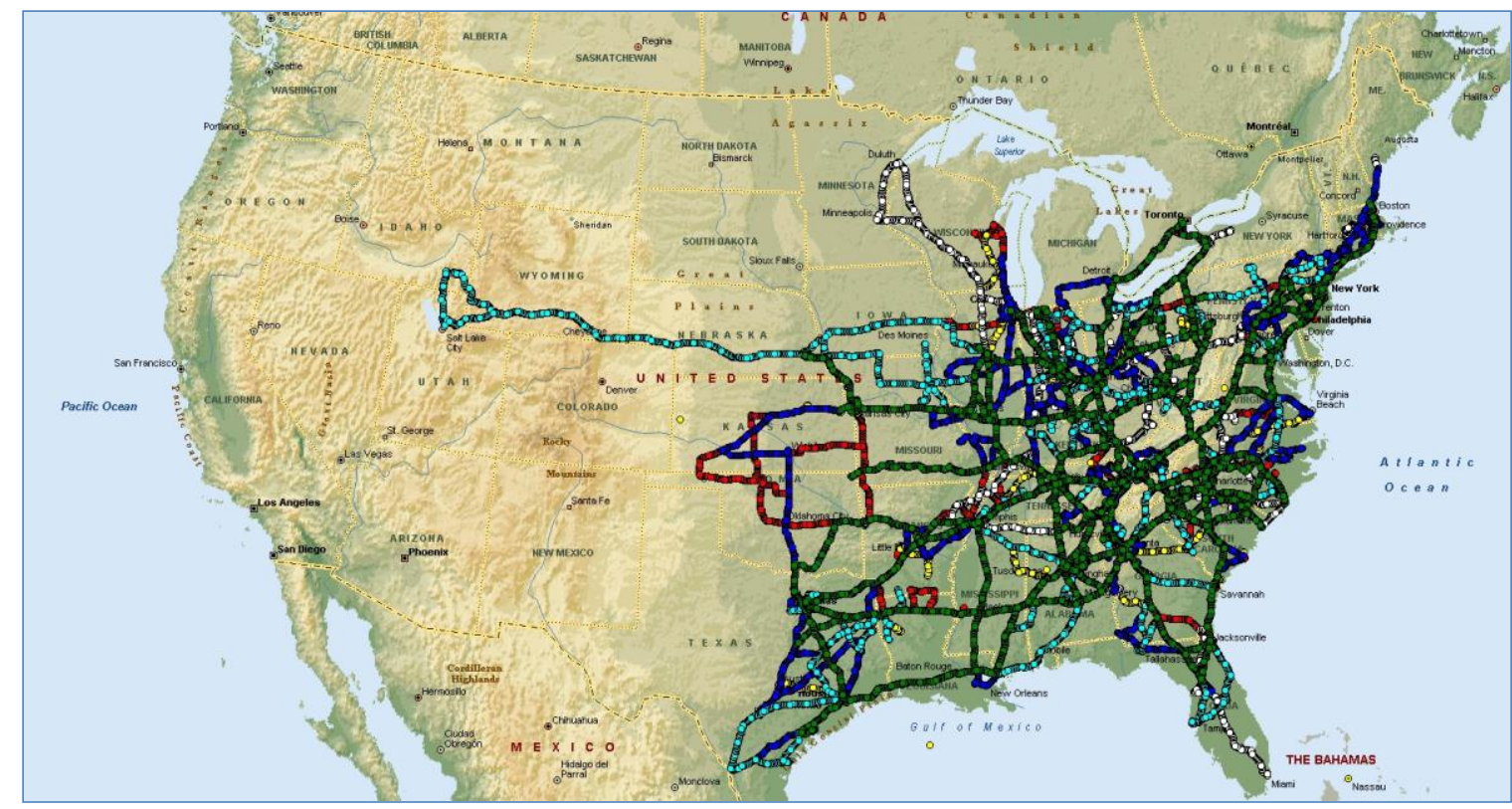

Fig. A1. Superimposed Trips (Six Class-8 Trucks, One Year)

Table A1. General Statistics for the Instrumented Tractors

\begin{tabular}{|c|c|c|c|c|c|c|c|}
\hline Statistics & $\begin{array}{c}\mathrm{T} 1 \\
\left(M^{1}-S^{A}\right)\end{array}$ & $\begin{array}{c}T^{2} \\
\left(M^{1}-D^{B}\right)\end{array}$ & $\begin{array}{c}\text { T3 } \\
\left(A^{2}-S^{A}\right)\end{array}$ & $\begin{array}{c}\mathrm{T4} \\
\left(M^{1}-D^{B}\right)\end{array}$ & $\begin{array}{c}\text { T5 } \\
\left(A^{2}-S^{A}\right)\end{array}$ & $\begin{array}{c}\mathrm{T6} \\
\left(\mathrm{A}^{2}-D^{\mathrm{B}}\right)\end{array}$ & $\begin{array}{l}\text { Grand } \\
\text { Total }\end{array}$ \\
\hline Distance Traveled [miles] & 106,891 & 114,095 & 117,355 & 124,917 & 127,626 & 97,417 & 688,302 \\
\hline $\begin{array}{l}\text { Total Time for Which Data } \\
\text { was Collected }{ }^{3}[\mathrm{hrs}]\end{array}$ & 3,783 & 4,451 & 3,779 & 4,413 & 4,281 & 3,067 & 23,774 \\
\hline Avg. Speed ${ }^{4}[\mathrm{mph}]$ & 28.26 & 25.63 & 31.05 & 28.31 & 29.81 & 31.76 & 28.95 \\
\hline Total Fuel Consumed [gal] & 15,982 & 16,701 & 16,805 & 19,361 & 18,494 & 15,995 & 103,336 \\
\hline $\begin{array}{l}\text { Overall Fuel Efficiency [mpg] } \\
\text { (from the Databus Sensor) }\end{array}$ & 6.69 & 6.83 & 6.98 & 6.45 & 6.90 & 6.09 & 6.66 \\
\hline
\end{tabular}

${ }^{1}$ Manual Transmission; ${ }^{2}$ Automatic Transmission; ${ }^{3}$ Includes Idling times; ${ }^{3} 4$ Distance Traveled/Total Time for Which Data Was Collected (includes idling times); ${ }^{A}$ New Generation Single Wide Base Tires; ${ }^{\mathrm{B}}$ Dual Tires 

APPENDIX B.

HTDC DATA CHANNELS COLLECTED 



\section{APPENDIX B. HTDC DATA CHANNELS COLLECTED}

Table B1 Data Channels and Sensors

\begin{tabular}{|c|c|c|}
\hline & Channel & Sensor \\
\hline 1 & Instantaneous Fuel Economy & J1939 Vehicle Data Bus \\
\hline 2 & Average Fuel Economy & J1939 Vehicle Data Bus \\
\hline 3 & Fuel Rate & J1939 Vehicle Data Bus \\
\hline 4 & Total Fuel Used & J1939 Vehicle Data Bus \\
\hline 5 & Total Idle Fuel Used & J1939 Vehicle Data Bus \\
\hline 6 & Current Gear & J1939 Vehicle Data Bus \\
\hline 7 & Selected Gear & J1939 Vehicle Data Bus \\
\hline 8 & Output Shaft Speed & J1939 Vehicle Data Bus \\
\hline 9 & Actual Engine - Percent Torque & J1939 Vehicle Data Bus \\
\hline 10 & Actual Gear Ratio & J1939 Vehicle Data Bus \\
\hline 11 & $\begin{array}{l}\text { Driver's Demand Engine - Percent } \\
\text { Torque }\end{array}$ & J1939 Vehicle Data Bus \\
\hline 12 & Percent Load at Current Speed & J1939 Vehicle Data Bus \\
\hline 13 & Engine Speed & J1939 Vehicle Data Bus \\
\hline 14 & Engine Oil Temperature & J1939 Vehicle Data Bus \\
\hline 15 & Fuel Temperature & J1939 Vehicle Data Bus \\
\hline 16 & Front Axle Speed & J1939 Vehicle Data Bus \\
\hline 17 & High Resolution Total Vehicle Distance & J1939 Vehicle Data Bus \\
\hline 18 & Accelerator Pedal Position & J1939 Vehicle Data Bus \\
\hline 19 & Total Idle Hours & J1939 Vehicle Data Bus \\
\hline 20 & Maximum Vehicle Speed Limit & J1939 Vehicle Data Bus \\
\hline 21 & Total Power Takeoff Hours & J1939 Vehicle Data Bus \\
\hline 22 & Clutch Switch & J1939 Vehicle Data Bus \\
\hline 23 & Brake Switch & J1939 Vehicle Data Bus \\
\hline 24 & Cruise Control Enable Switch & J1939 Vehicle Data Bus \\
\hline 25 & Cruise Control Active & J1939 Vehicle Data Bus \\
\hline 26 & Cruise Control Accelerate Switch & J1939 Vehicle Data Bus \\
\hline 27 & Cruise Control Resume Switch & J1939 Vehicle Data Bus \\
\hline 28 & Cruise Control Coast Switch & J1939 Vehicle Data Bus \\
\hline 29 & Cruise Control Set Switch & J1939 Vehicle Data Bus \\
\hline 30 & Battery Electrical Potential (Voltage) & J1939 Vehicle Data Bus \\
\hline 31 & Tractor Drive Axles Weight & Air-Weigh \\
\hline 32 & Tractor Steer Axle Weight & Air-Weigh \\
\hline 33 & Trailer Weight & Air-Weigh \\
\hline 34 & Tractor Serial Number & Air-Weigh \\
\hline 35 & Trailer Serial Number & Air-Weigh \\
\hline 36 & Latitude & VBOX \\
\hline 37 & Longitude & VBOX \\
\hline
\end{tabular}




\begin{tabular}{|c|c|c|}
\hline & Channel & Sensor \\
\hline 38 & Altitude & VBOX \\
\hline 39 & Vertical velocity & VBOX \\
\hline 40 & Velocity over ground & VBOX \\
\hline 41 & Longitudinal acceleration & VBOX \\
\hline 42 & Lateral acceleration & VBOX \\
\hline 43 & Heading & VBOX \\
\hline 44 & Satellites & VBOX \\
\hline 45 & Time UTC & VBOX \\
\hline 46 & Wind Speed & Weather Station \\
\hline 47 & Wind Direction & Weather Station \\
\hline 48 & Rain Intensity & Weather Station \\
\hline 49 & Barometric Pressure & Weather Station \\
\hline 50 & Air Temperature & Weather Station \\
\hline 51 & Relative Humidity & Weather Station \\
\hline 52 & Tire Flag & Calculated from AirWeigh data \\
\hline 53 & Road Grade & Calculated from VBOX data \\
\hline 54 & Stationary Latitude & Calculated from VBOX data \\
\hline 55 & Stationary Longitude & Calculated from VBOX data \\
\hline 56 & Tractor-Trailer Marriage & Calculated from AirWeigh data \\
\hline
\end{tabular}

Table B2 Additional Information Added to Each Record Collected

\begin{tabular}{rll}
\hline \multicolumn{2}{c}{ Channel } & \multicolumn{1}{c}{ Source } \\
\hline $\mathbf{1}$ & Location (Urban/Rural) & Post-processed Information \\
\hline $\mathbf{2}$ & Location (City) & Post-processed Information \\
$\mathbf{3}$ & Location (State) & Post-processed Information \\
$\mathbf{4}$ & Roadway Facility (Freeway/Surface Street) & Post-processed Information \\
\hline $\mathbf{5}$ & Freeway ID & Post-processed Information \\
$\mathbf{6}$ & Roadway Grade & Post-processed Information (derived from VBOX data) \\
$\mathbf{7}$ & Truck Total Weight & Post-processed Information \\
\hline $\mathbf{8}$ & Time of Day & Post-processed Information (corrected for Time Zone) \\
\hline $\mathbf{9}$ & Driver ID & Integration with Carrier DB \\
\hline $\mathbf{1 0}$ & Type of Tires & Integration with Carrier DB \\
\hline *The driver ID field does not contain personal information; it is just a number that is always assigned to the same driver
\end{tabular}




\section{APPENDIX C. \\ EFFECT OF WEIGHT AND ROADWAY GRADE ON THE FUEL ECONOMY OF CLASS-8 FREIGHT TRUCKS (INTERIM REPORT - SEPTEMBER 2010)}





\section{APPENDIX C. EFFECT OF WEIGHT AND ROADWAY GRADE ON THE FUEL ECONOMY OF CLASS-8 FREIGHT TRUCKS (INTERIM REPORT - SEPTEMBER 2010)}

\section{Background}

In 2007 and 2008, the Oak Ridge National Laboratory (ORNL), in partnership with several industry partners, collected real-world performance and situational data for long-haul operations of Class- 8 trucks from a fleet engaged in normal freight operations. Six Class-8 trucks from a selected fleet, which operates within a large area of the country extending from the east coast to the Mountain Time Zone and from Canada to the US-Mexican border (see Appendix A), were instrumented to collect 56 channels of data for over a year at a rate of $5 \mathrm{~Hz}$ (or 5 readings per second) using an ORNL-developed data acquisition system (DAS). Those channels included information such as instantaneous fuel rate, engine speed, gear ratio, vehicle speed, and other information read from the vehicle's databus; weather information (wind speed, precipitation, air temperature, etc.) gathered from an on-board weather station; spatial information (latitude, longitude, altitude) acquired from a GPS (Global Positioning System) device; and instantaneous tractor and trailer weight obtained from devices mounted on the six participating tractors and ten trailers (see Appendix B for more details). In addition to the data collected directly from the on-board sensors, ORNL added other information to the database that was obtained from the carrier and by post processing the raw data. This included type of roadway (freeways or surface streets), spatial location specificity (urban or rural areas), roadway grade, type of tires, driver ID, and others. Three of the six instrumented tractors and five of the ten instrumented trailers were mounted with new generation single wide-based tires and the others were mounted with new regular dual tires. Over the duration of the project, the six tractors traveled nearly 700,000 miles collecting over $295 \mathrm{~GB}$ of uncompressed data.

\section{Study Methodology}

For the present study, the information contained in the HTDC database was parsed by type of terrain, tractor/trailer tire type, and vehicle speed; and the fuel efficiency was subsequently computed. The type of terrain was divided into down-sloping terrain (road grade less than-0.2\%), flat terrain (road grade between $-0.2 \%$ and $0.2 \%$ ), and upslope terrain (road grade greater than $0.2 \%)$. The database contains information about the type of tires that were mounted on the participating vehicles; i.e., regular dual tires (duals) or new generation wide-based single tires (singles). Four categories are possible for the tractor/trailer tire combination (i.e., duals-duals, duals-singles, singles-duals, and singles-singles).

In the first part of this study, two vehicle speeds were considered in the computation of Class-8 truck fuel efficiency, $55 \mathrm{mph}$ (i.e., vehicle speeds between 54.5 and $55.5 \mathrm{mph}$ ) and $65 \mathrm{mph}$ (i.e., 
vehicle speeds between 64.5 and $65.5 \mathrm{mph}$ ). For the analysis, the four tractor-trailer tire combinations were considered. In the second part of the study, the fuel efficiency was computed for any speed between 0 and $75 \mathrm{mph}$, but no consideration was given to the type of tires (i.e., the information was not disaggregated by tractor/trailer tire combination). The fuel efficiencies were computed as the ratio of the distance traveled divided by the total fuel consumed for each one of the categories considered in this study.

\section{Results}

Tables 1 to 6 below show the results of the first analysis. Tables 1 and 2 compare the fuel efficiencies obtained at the two vehicle speeds: 55 and $65 \mathrm{mph}$, for vehicles mounted with dual tires on the tractor and duals or singles on the trailer. Tables 3 and 4 present the same type of information, but for the case in which the tractor was mounted with singles. Notice that in all cases, the fuel efficiency obtained at $65 \mathrm{mph}$ is always higher than the fuel efficiency obtained at $55 \mathrm{mph}$. One likely explanation for this is that new Class-8 vehicles (the vehicles participating in this project were all Volvos 2005) are optimized for $65 \mathrm{mph}$ highway speeds. As can be observed when comparing Table 1 vs. Table 2 and Table 3 vs. Table 4, the distance traveled by the participant vehicles at a speed of $65 \mathrm{mph}$ was four -times the distance they traveled at a speed of $55 \mathrm{mph}$. The information presented in the last horizontal section of Tables 1 to 4 (i.e., Duals-Any and Singles-Any) is also shown graphically in Figure 1.

Tables 5 and 6 present the information shown in Tables 1 to 4 in an aggregated form where all the tractor/trailer tire combinations have been collapsed into one category (i.e., Any-Any). This information is also presented in Figure 2.

Table 1. Class-8 Truck Fuel Efficiency (FE) by Terrain Type and Tractor-Trailer Tires (Duals-Any) Speed 55 mph (54.5 to $55.5 \mathrm{mph}$ ) - Any Vehicle Weight

\begin{tabular}{|c|c|c|r|r|r|r|}
\hline $\begin{array}{c}\text { Tractor } \\
\text { Tires }\end{array}$ & $\begin{array}{c}\text { Trailer } \\
\text { Tires }\end{array}$ & $\begin{array}{c}\text { Road Grade } \\
\text { Type }\end{array}$ & $\begin{array}{c}\text { Distance } \\
\text { [miles] }\end{array}$ & $\begin{array}{c}\text { Fuel Used } \\
\text { [gal] }\end{array}$ & $\begin{array}{c}\text { FE } \\
\text { [mpg] }\end{array}$ & $\begin{array}{c}\text { Road Grade } \\
\text { Type } \\
\text { Distribution }\end{array}$ \\
\hline \multirow{3}{*}{ Duals } & \multirow{2}{*}{ Singles } & Down Slope & 543.2 & 43.4 & 12.505 & $35.93 \%$ \\
& & Flat & 197.1 & 26.0 & 7.592 & $13.03 \%$ \\
& & Up Slope & 771.6 & 164.9 & 4.680 & $51.04 \%$ \\
\cline { 3 - 7 } & & Overall & $1,511.9$ & 234.3 & 6.454 & $100.00 \%$ \\
\hline \hline \multirow{3}{*}{ Duals } & \multirow{2}{*}{ Duals } & Down Slope & 946.2 & 82.3 & 11.501 & $34.80 \%$ \\
& & Flat & 380.9 & 57.1 & 6.671 & $14.01 \%$ \\
& & Up Slope & $1,391.7$ & 323.9 & 4.297 & $51.19 \%$ \\
\cline { 3 - 7 } & & Overall & $2,718.8$ & 463.2 & 5.869 & $100.00 \%$ \\
\hline \hline \multirow{3}{*}{ Duals } & \multirow{2}{*}{ Any } & Down Slope & $1,489.4$ & 125.7 & 11.848 & $35.20 \%$ \\
& & Flat & 577.9 & 83.1 & 6.958 & $13.66 \%$ \\
& & Up Slope & $2,163.3$ & 488.7 & 4.426 & $51.13 \%$ \\
\cline { 3 - 7 } & & Overall & $4,230.6$ & 697.5 & 6.065 & $100.00 \%$ \\
\hline
\end{tabular}


Table 2. Class-8 Truck Fuel Efficiency (FE) by Terrain Type and Tractor-Trailer Tires (Duals-Any) Speed $65 \mathrm{mph}$ (64.5 to $65.5 \mathrm{mph}$ ) - Any Vehicle Weight

\begin{tabular}{|c|c|c|r|r|r|r|}
\hline $\begin{array}{c}\text { Tractor } \\
\text { Tires }\end{array}$ & $\begin{array}{c}\text { Trailer } \\
\text { Tires }\end{array}$ & $\begin{array}{c}\text { Road Grade } \\
\text { Type }\end{array}$ & $\begin{array}{c}\text { Distance } \\
\text { [miles] }\end{array}$ & $\begin{array}{c}\text { Fuel Used } \\
\text { [gal] }\end{array}$ & $\begin{array}{c}\text { FE } \\
\text { [mpg] }\end{array}$ & $\begin{array}{c}\text { Road Grade } \\
\text { Type } \\
\text { Distribution }\end{array}$ \\
\hline \multirow{3}{*}{ Duals } & \multirow{3}{*}{ Singles } & Down Slope & $2,198.1$ & 146.8 & 14.973 & $40.49 \%$ \\
& & Flat & 841.2 & 108.0 & 7.792 & $15.50 \%$ \\
& & Up Slope & $2,388.9$ & 480.1 & 4.976 & $44.01 \%$ \\
\cline { 3 - 7 } & & Overall & $5,428.2$ & 734.9 & 7.387 & $100.00 \%$ \\
\hline \hline \multirow{3}{*}{ Duals } & \multirow{3}{*}{ Duals } & Down Slope & $4,031.3$ & 296.5 & 13.597 & $37.82 \%$ \\
& & Flat & $1,733.5$ & 235.9 & 7.349 & $16.26 \%$ \\
\cline { 3 - 7 } & & Overall & $10,660.5$ & $1,571.1$ & 6.785 & $100.00 \%$ \\
\hline \hline \multirow{3}{*}{ Duals } & \multirow{2}{*}{ Any } & Down Slope & $6,229.4$ & 443.3 & 14.053 & $38.72 \%$ \\
& & Flat & $2,574.8$ & 343.8 & 7.488 & $16.00 \%$ \\
& & Up Slope & $7,284.6$ & $1,518.9$ & 4.796 & $45.28 \%$ \\
\cline { 3 - 7 } & & Overall & $16,088.7$ & $2,306.0$ & 6.977 & $100.00 \%$ \\
\hline
\end{tabular}

Table 3. Class-8 Truck Fuel Efficiency (FE) by Terrain Type and Tractor-Trailer Tires (Singles-Any) Speed 55 mph (54.5 to $55.5 \mathrm{mph}$ ) - Any Vehicle Weight

\begin{tabular}{|c|c|c|r|r|r|r|}
\hline $\begin{array}{c}\text { Tractor } \\
\text { Tires }\end{array}$ & $\begin{array}{c}\text { Trailer } \\
\text { Tires }\end{array}$ & $\begin{array}{c}\text { Road Grade } \\
\text { Type }\end{array}$ & $\begin{array}{c}\text { Distance } \\
\text { [miles] }\end{array}$ & $\begin{array}{c}\text { Fuel Used } \\
\text { [gal] }\end{array}$ & $\begin{array}{c}\text { FE } \\
\text { [mpg] }\end{array}$ & $\begin{array}{c}\text { Road Grade } \\
\text { Type } \\
\text { Distribution }\end{array}$ \\
\hline \multirow{3}{*}{ Singles } & \multirow{3}{*}{ Singles } & Down Slope & 395.6 & 30.9 & 12.784 & $36.43 \%$ \\
& & Flat & 125.5 & 16.3 & 7.721 & $11.56 \%$ \\
& & Up Slope & 564.8 & 128.5 & 4.396 & $52.01 \%$ \\
\cline { 3 - 7 } & & Overall & $1,085.9$ & 175.7 & 6.181 & $100.00 \%$ \\
\hline \hline \multirow{3}{*}{ Singles } & \multirow{3}{*}{ Duals } & Down Slope & $1,038.8$ & 81.8 & 12.700 & $37.29 \%$ \\
& & Flat & 297.8 & 38.3 & 7.779 & $10.69 \%$ \\
& & Up Slope & $1,449.1$ & 333.2 & 4.349 & $52.02 \%$ \\
\cline { 3 - 7 } & & Overall & $2,785.6$ & 453.2 & 6.146 & $100.00 \%$ \\
\hline \hline \multirow{3}{*}{ Singles } & \multirow{3}{*}{ Any } & Down Slope & $1,434.4$ & 112.7 & 12.723 & $37.05 \%$ \\
& & Flat & 423.3 & 54.5 & 7.762 & $10.93 \%$ \\
& & Up Slope & $2,013.8$ & 461.6 & 4.362 & $52.02 \%$ \\
\cline { 3 - 7 } & & Overall & $3,871.5$ & 628.9 & 6.156 & $100.00 \%$ \\
\hline
\end{tabular}


Table 4. Class-8 Truck Fuel Efficiency (FE) by Terrain Type and Tractor-Trailer Tires (Singles-Any) Speed $65 \mathrm{mph}$ (64.5 to $65.5 \mathrm{mph}$ ) - Any Vehicle Weight

\begin{tabular}{|c|c|c|r|r|r|r|}
\hline $\begin{array}{c}\text { Tractor } \\
\text { Tires }\end{array}$ & $\begin{array}{c}\text { Trailer } \\
\text { Tires }\end{array}$ & $\begin{array}{c}\text { Road Grade } \\
\text { Type }\end{array}$ & $\begin{array}{c}\text { Distance } \\
\text { [miles] }\end{array}$ & $\begin{array}{c}\text { Fuel Used } \\
\text { [gal] }\end{array}$ & $\begin{array}{c}\text { FE } \\
\text { [mpg] }\end{array}$ & $\begin{array}{c}\text { Road Grade } \\
\text { Type } \\
\text { Distribution }\end{array}$ \\
\hline \multirow{3}{*}{ Singles } & \multirow{3}{*}{ Singles } & Down Slope & $1,463.3$ & 104.2 & 14.037 & $38.30 \%$ \\
& & Flat & 549.7 & 66.9 & 8.213 & $14.39 \%$ \\
& & Up Slope & $1,807.7$ & 361.5 & 5.000 & $47.31 \%$ \\
\cline { 3 - 7 } & & Overall & $3,820.6$ & 532.7 & 7.172 & $100.00 \%$ \\
\hline \hline \multirow{3}{*}{ Singles } & \multirow{3}{*}{ Duals } & Down Slope & $3,429.3$ & 247.3 & 13.864 & $39.19 \%$ \\
& & Flat & $1,069.6$ & 135.0 & 7.923 & $12.22 \%$ \\
& & Up Slope & $4,251.0$ & 876.5 & 4.850 & $48.58 \%$ \\
\cline { 3 - 7 } & & Overall & $8,750.0$ & $1,258.9$ & 6.951 & $100.00 \%$ \\
\hline \hline \multirow{3}{*}{ Singles } & \multirow{3}{*}{ Any } & Down Slope & $4,892.6$ & 351.6 & 13.916 & $38.92 \%$ \\
& & Flat & $1,619.3$ & 201.9 & 8.019 & $12.88 \%$ \\
& & Up Slope & $6,058.7$ & $1,238.0$ & 4.894 & $48.20 \%$ \\
\cline { 3 - 7 } & & Overall & $12,570.6$ & $1,791.5$ & 7.017 & $100.00 \%$ \\
\hline
\end{tabular}

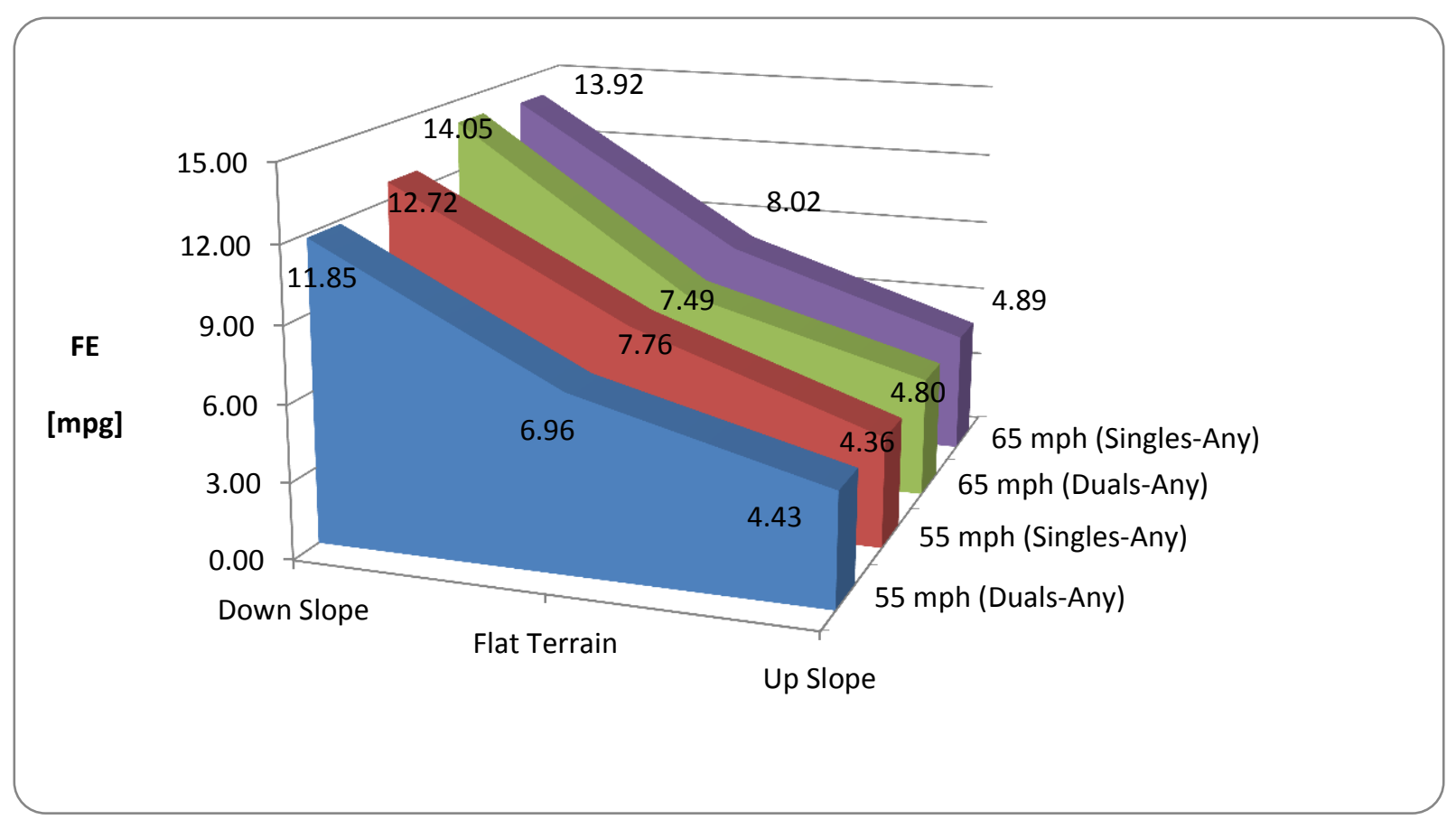

Figure 1. Class-8 Truck Fuel Efficiency (FE) by Type of Terrain, Speed and Type of Tires (Any Vehicle Weight) 
Table 5. Class-8 Truck Fuel Efficiency (FE) by Terrain Type Speed $55 \mathrm{mph}$ (54.5 to $55.5 \mathrm{mph}$ ) - Any Vehicle Weight

\begin{tabular}{|c|c|c|r|r|r|r|}
\hline $\begin{array}{c}\text { Tractor } \\
\text { Tires }\end{array}$ & $\begin{array}{c}\text { Trailer } \\
\text { Tires }\end{array}$ & $\begin{array}{c}\text { Road Grade } \\
\text { Type }\end{array}$ & $\begin{array}{c}\text { Distance } \\
\text { [miles] }\end{array}$ & $\begin{array}{c}\text { Fuel Used } \\
\text { [gal] }\end{array}$ & $\begin{array}{c}\text { FE } \\
\text { [mpg] }\end{array}$ & $\begin{array}{c}\text { Road Grade } \\
\text { Type } \\
\text { Distribution }\end{array}$ \\
\hline \multirow{3}{*}{ Any } & \multirow{2}{*}{ Any } & Down Slope & 938.8 & 74.4 & 12.621 & $36.14 \%$ \\
& & Flat & 322.6 & 42.2 & 7.641 & $12.42 \%$ \\
& & Up Slope & $1,336.3$ & 293.3 & 4.556 & $51.44 \%$ \\
\cline { 3 - 7 } & & Overall & $2,597.8$ & 409.9 & 6.337 & $100.00 \%$ \\
\hline
\end{tabular}

Table 6. Class-8 Truck Fuel Efficiency (FE) by Terrain Type Speed $65 \mathrm{mph}$ (64.5 to $65.5 \mathrm{mph}$ ) - Any Vehicle Weight

\begin{tabular}{|c|c|c|c|r|r|r|}
\hline $\begin{array}{c}\text { Tractor } \\
\text { Tires }\end{array}$ & $\begin{array}{c}\text { Trailer } \\
\text { Tires }\end{array}$ & $\begin{array}{c}\text { Road Grade } \\
\text { Type }\end{array}$ & $\begin{array}{c}\text { Distance } \\
\text { [miles] }\end{array}$ & $\begin{array}{c}\text { Fuel Used } \\
\text { [gal] }\end{array}$ & $\begin{array}{c}\text { FE } \\
\text { [mpg] }\end{array}$ & $\begin{array}{c}\text { Road Grade } \\
\text { Type } \\
\text { Distribution }\end{array}$ \\
\hline \multirow{3}{*}{ Any } & & Down Slope & $3,661.4$ & 251.0 & 14.585 & $39.59 \%$ \\
& \multirow{2}{*}{ Any } & Flat & $1,390.9$ & 174.9 & 7.953 & $15.04 \%$ \\
& & Up Slope & $4,196.5$ & 841.6 & 4.986 & $45.37 \%$ \\
\cline { 3 - 7 } & & Overall & $9,248.9$ & $1,267.6$ & 7.297 & $100.00 \%$ \\
\hline
\end{tabular}

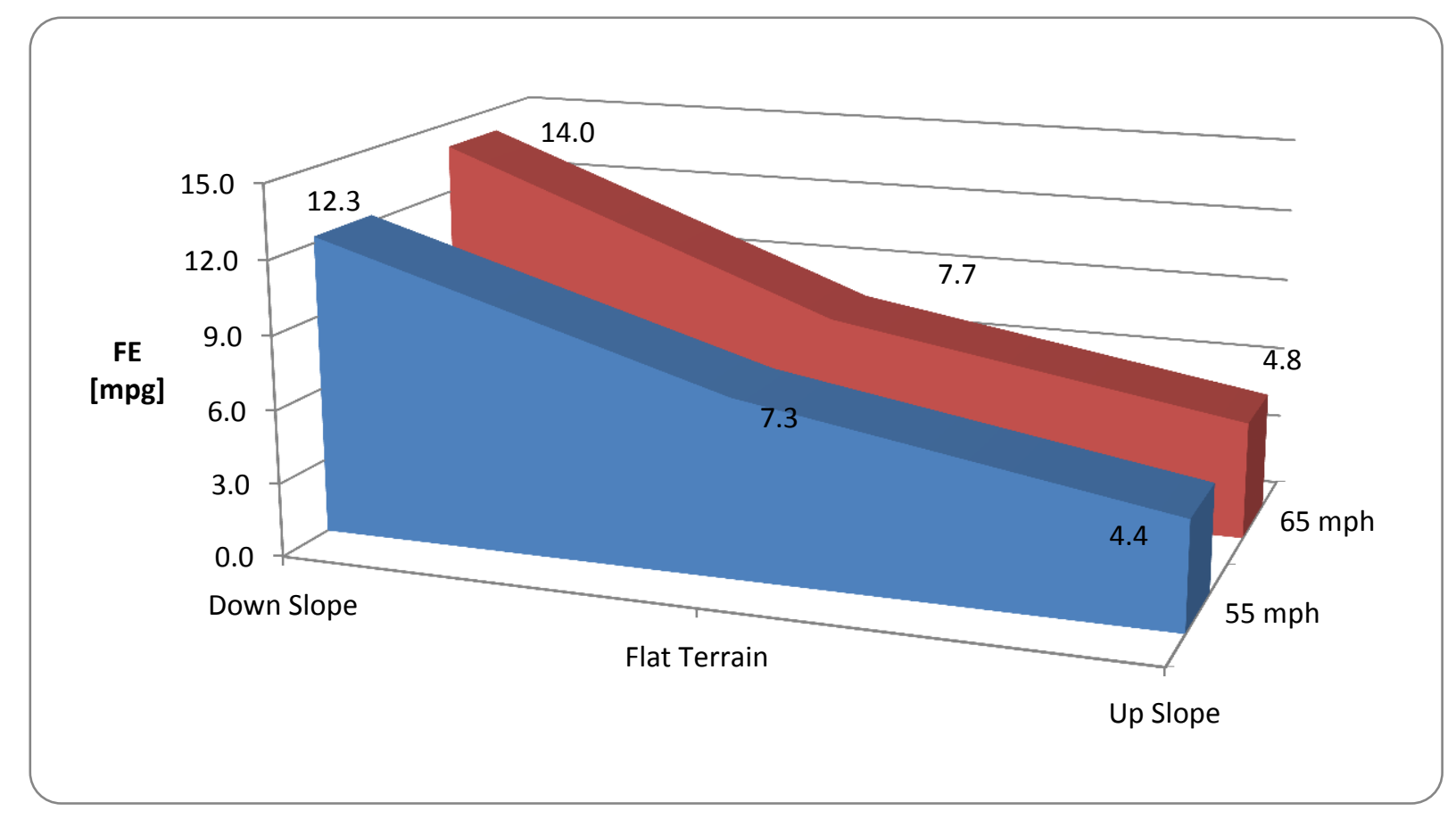

Figure 2. Class-8 Truck Fuel Efficiency (FE) by Type of Terrain and Speed (Any Tires, Any Vehicle Weight) 
In the second part of the study, the information in the HTDC database was parsed by speed and type of terrain, for any combination of tractor/trailer tires and vehicle weight; and the fuel efficiency was computed. Figures 3 to 8 show the information for Flat Terrain (Figures 3 and 4), Flat and Up Slope Terrain (Figures 5 and 6), and Down Slope Terrain (Figures 7 and 8). In all cases (Figures 3, 5, and 7) there are three distinct regions: an increasing fuel-efficiency region ( 0 to just over $55 \mathrm{mph}$ ) which is mostly a vehicle-acceleration region; a flat fuel-efficiency region (about 55-60 $\mathrm{mph}$ to $70 \mathrm{mph}$ ) in which the fuel efficiency is almost constant and which is mostly a vehicle-cruising region; and a high fuel-efficiency region (above $70 \mathrm{mph}$ ). The high fuel efficiencies are the result of the vehicles transitioning from a down-sloping terrain to the type of terrain that is shown in the figures (i.e., Flat, Flat and Upslope, and Down Slope Terrain type) thereby achieving high fuel efficiencies by using the inertia that the vehicle had and not needing to use fuel while entering the new terrain-type region.

Figures 4,6 , and 8 show the flat fuel-efficiency region is more detail. Consider, for example, Figure 4 (Flat Terrain). The fuel efficiency peaks at about 59-60 mph and then starts to decrease very slightly until about $70 \mathrm{mph}$ (i.e., the start of the third fuel-efficiency region). The same type of performance can be observed in Figure 6 (Flat and Upslope Terrain) and Figure 8 (Down Slope Terrain). Notice that if the third fuel efficiency region, which is mostly an artifact of the data processing, is eliminated, then the peak fuel efficiency under the conditions studied here correspond to speeds that are around $60 \mathrm{mph}$.

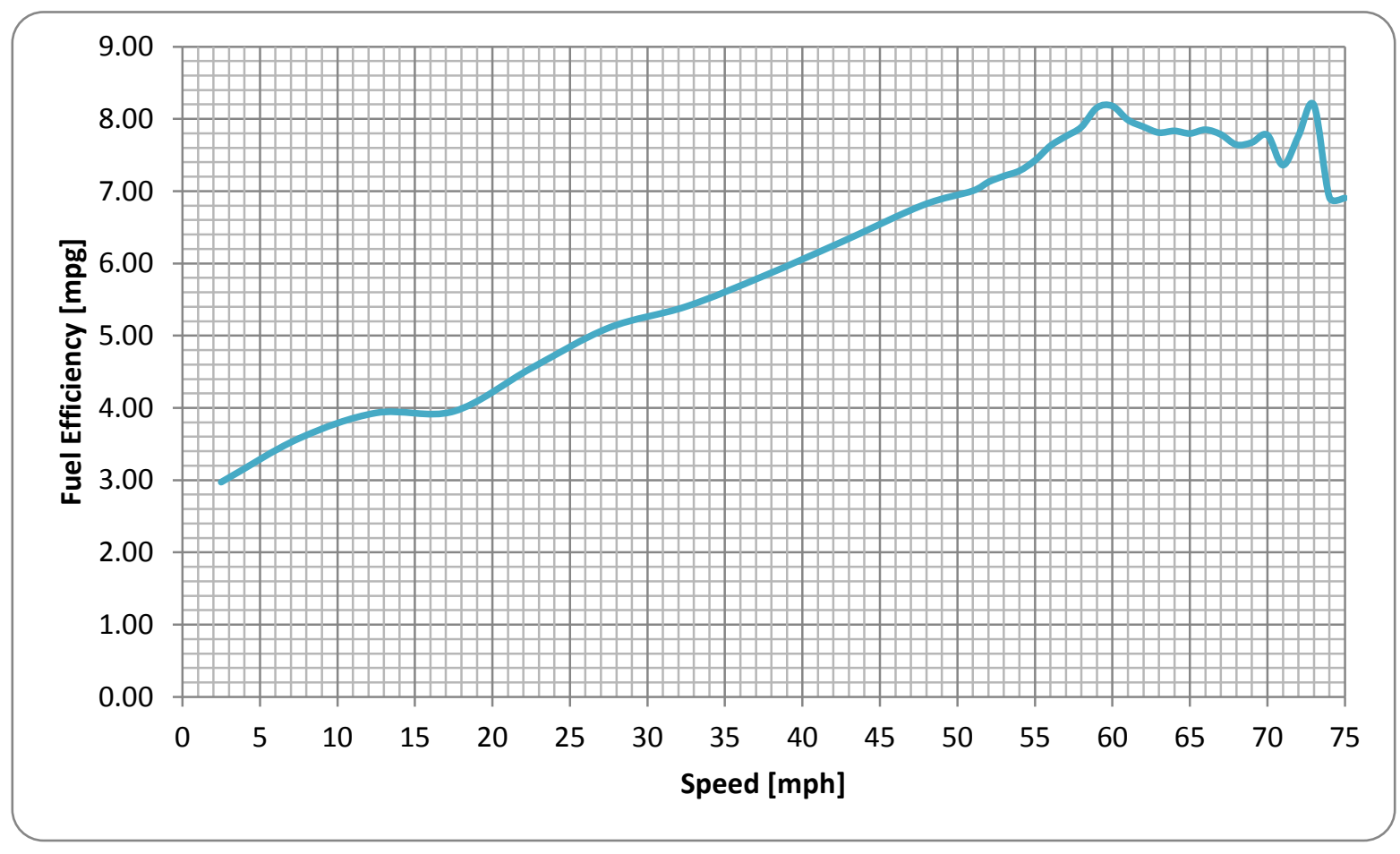

Figure 3. Class-8 Truck Fuel Efficiency by Speed ( 0 to $75 \mathrm{mph}$ ) Flat Terrain (Any Type of Tires, Any Vehicle Weight) 


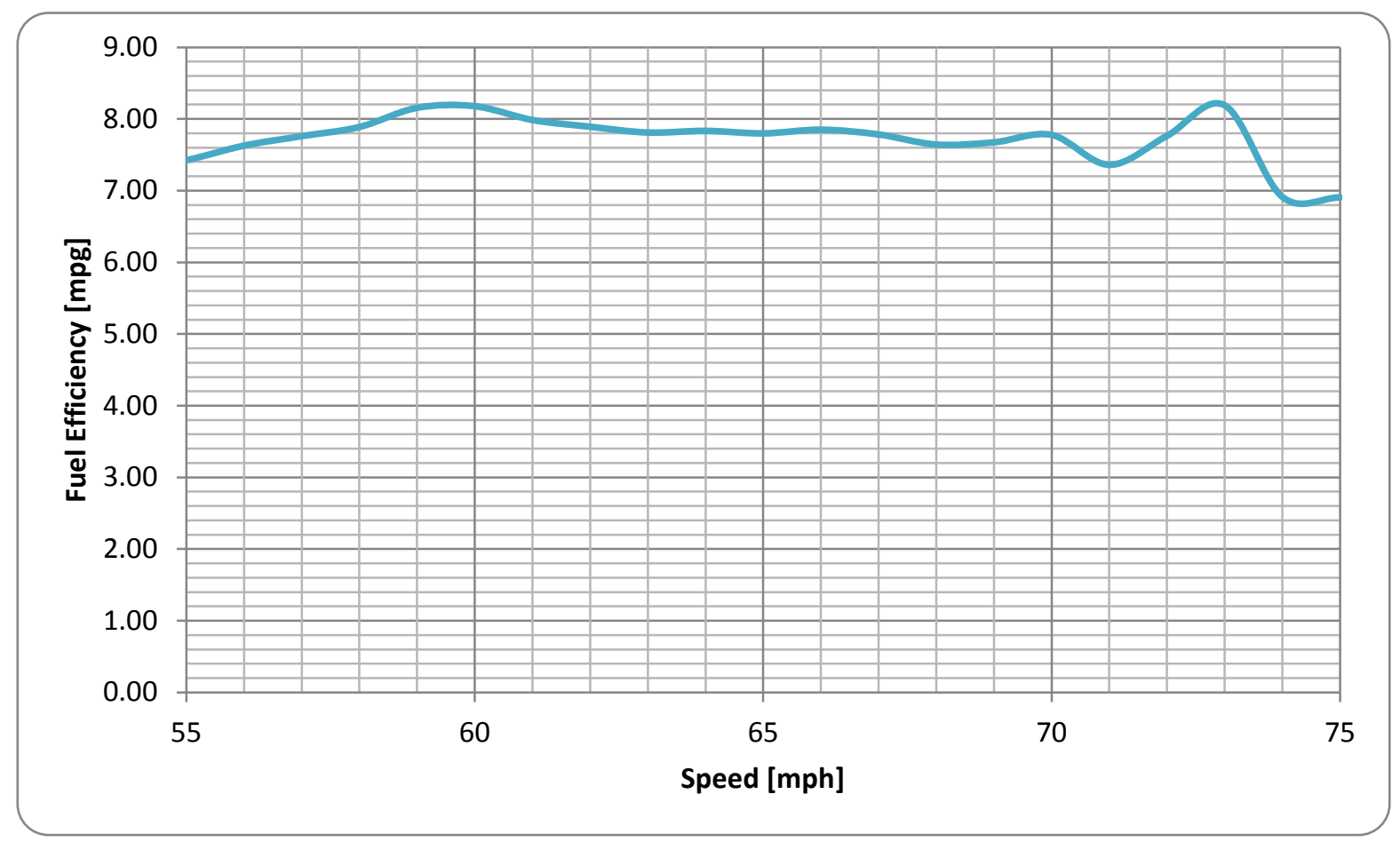

Figure 4. . Class-8 Truck Fuel Efficiency by Speed (55 to $75 \mathrm{mph}$ ) Flat Terrain (Any Type of Tires, Any Vehicle Weight)

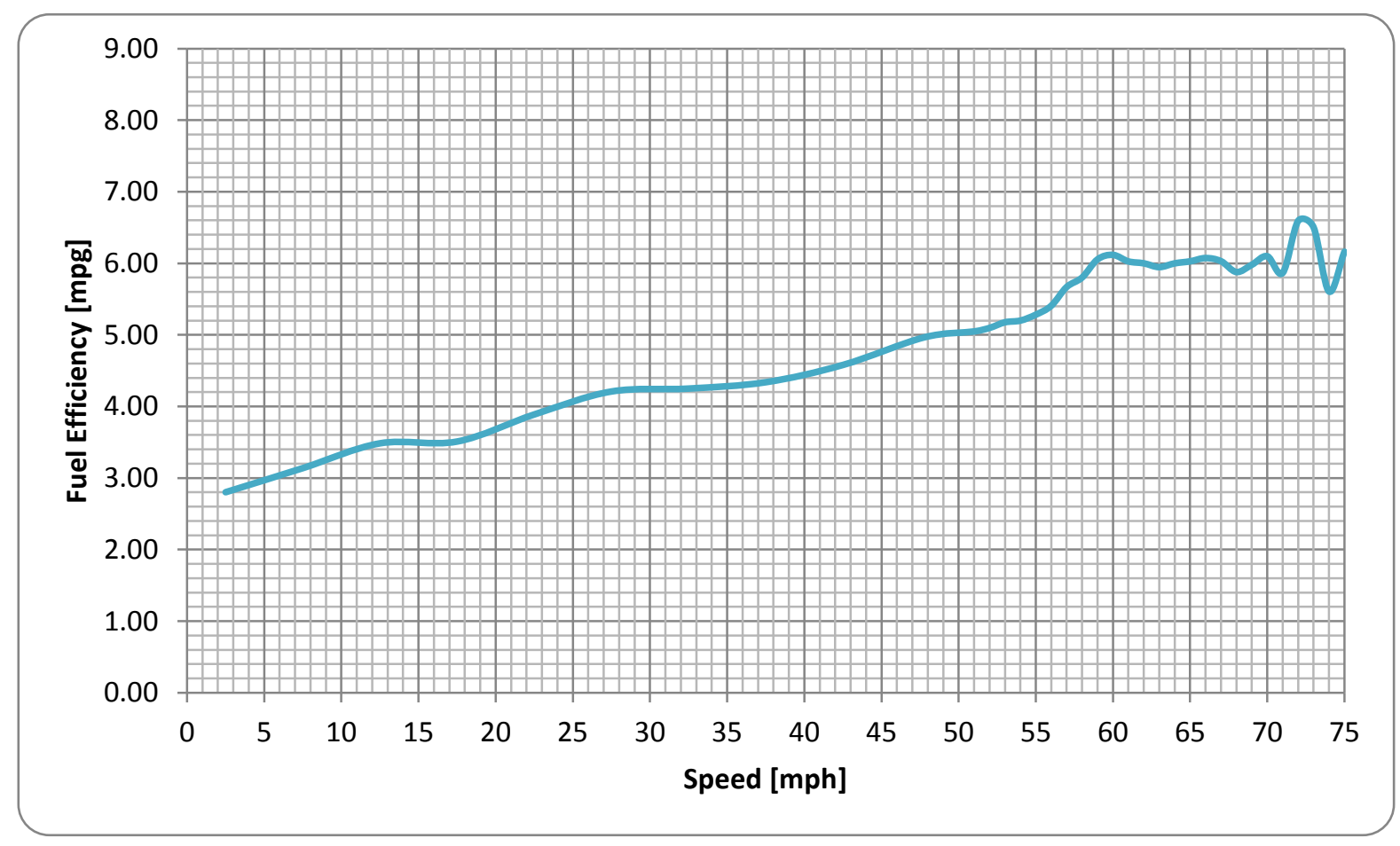

Figure 5. - Class-8 Truck Fuel Efficiency by Speed ( 0 to $75 \mathrm{mph}$ ) Flat and Upslope Terrain (Any Type of Tires, Any Vehicle Weight) 


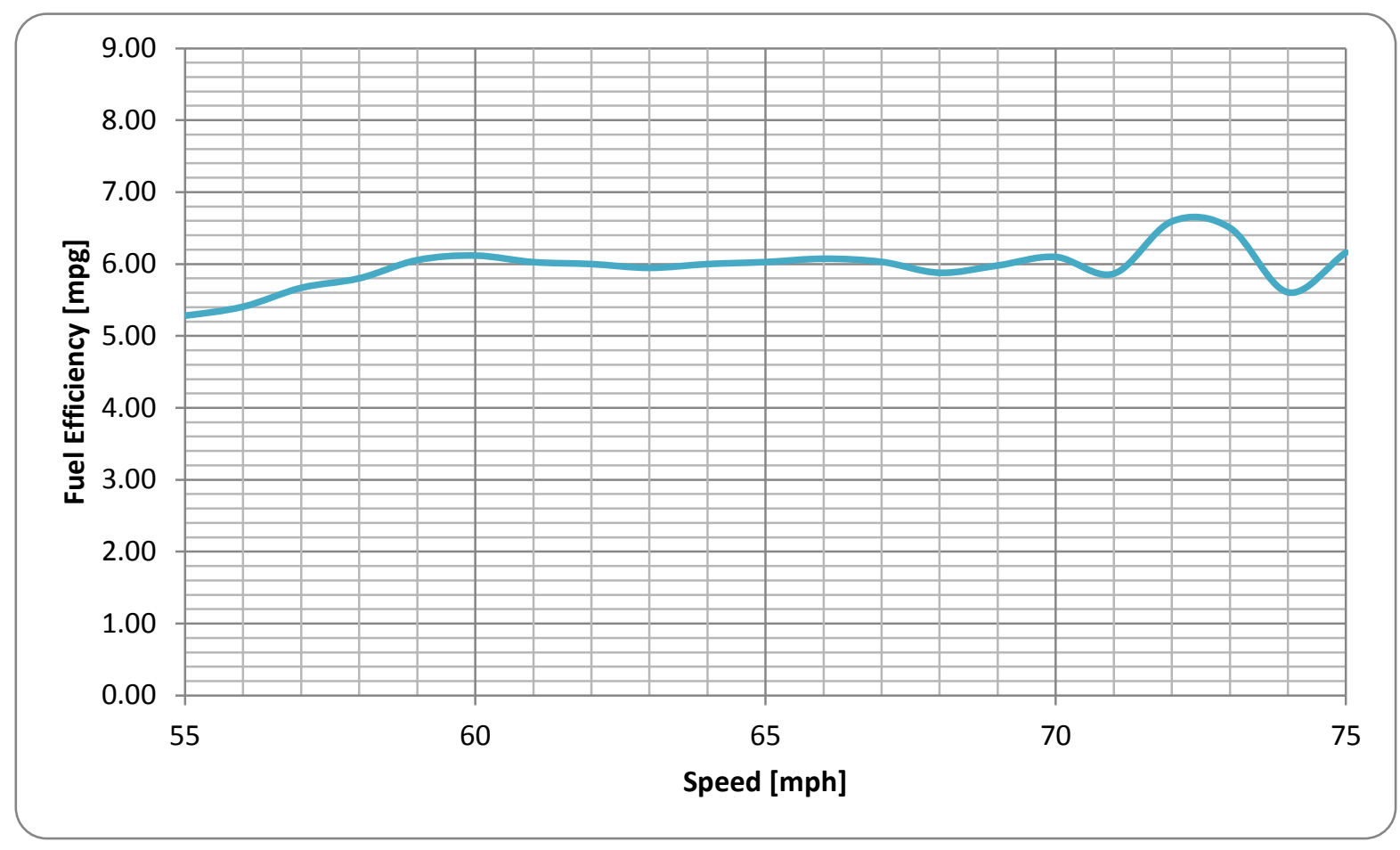

Figure 6. Class-8 Truck Fuel Efficiency by Speed ( 55 to $75 \mathrm{mph}$ )

Flat and Upslope Terrain (Any Type of Tires, Any Vehicle Weight)

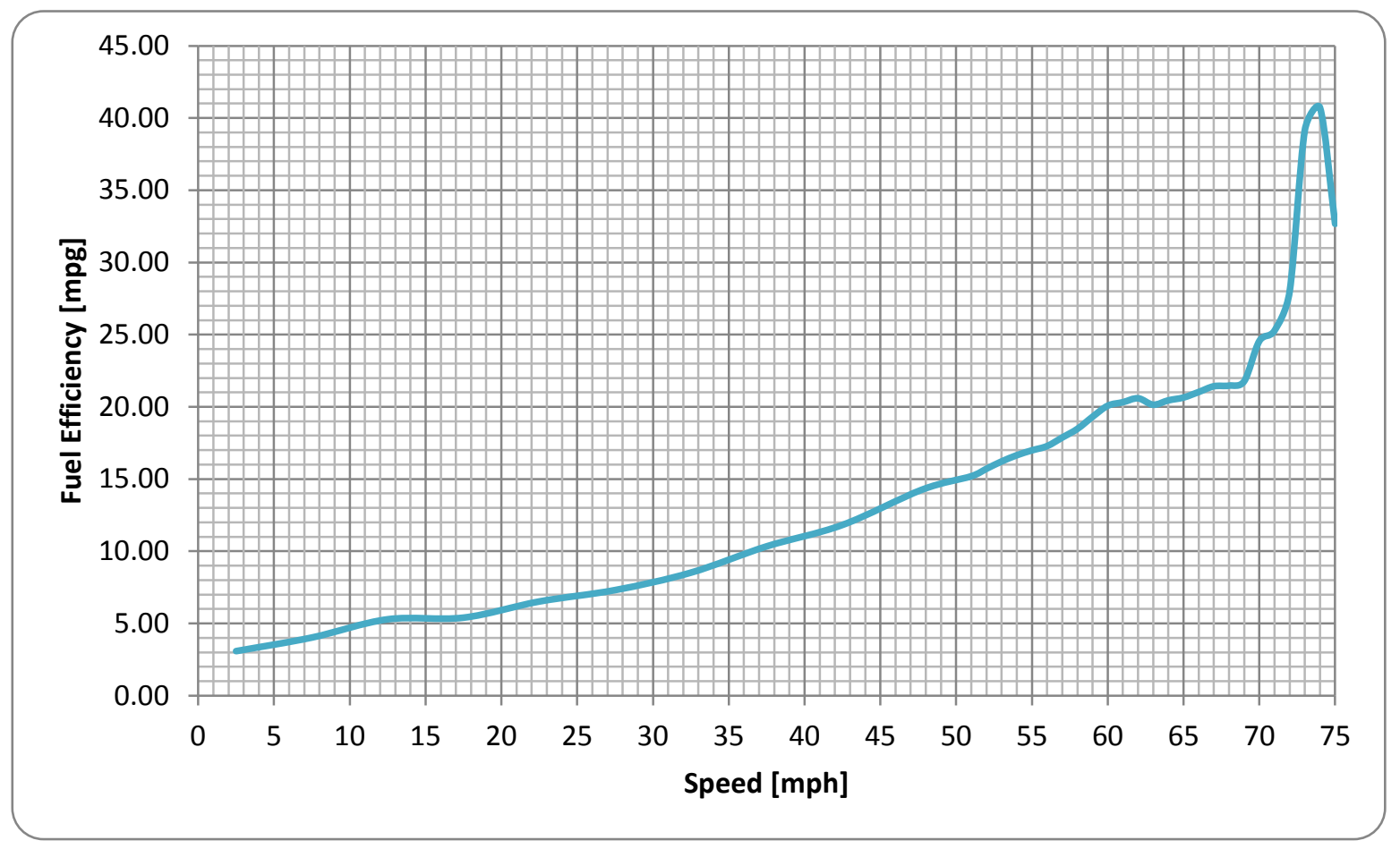

Figure 7. Class-8 Truck Fuel Efficiency by Speed (0 to $75 \mathrm{mph}$ ) Down Slope Terrain (Any Type of Tires, Any Vehicle Weight)

C-10 


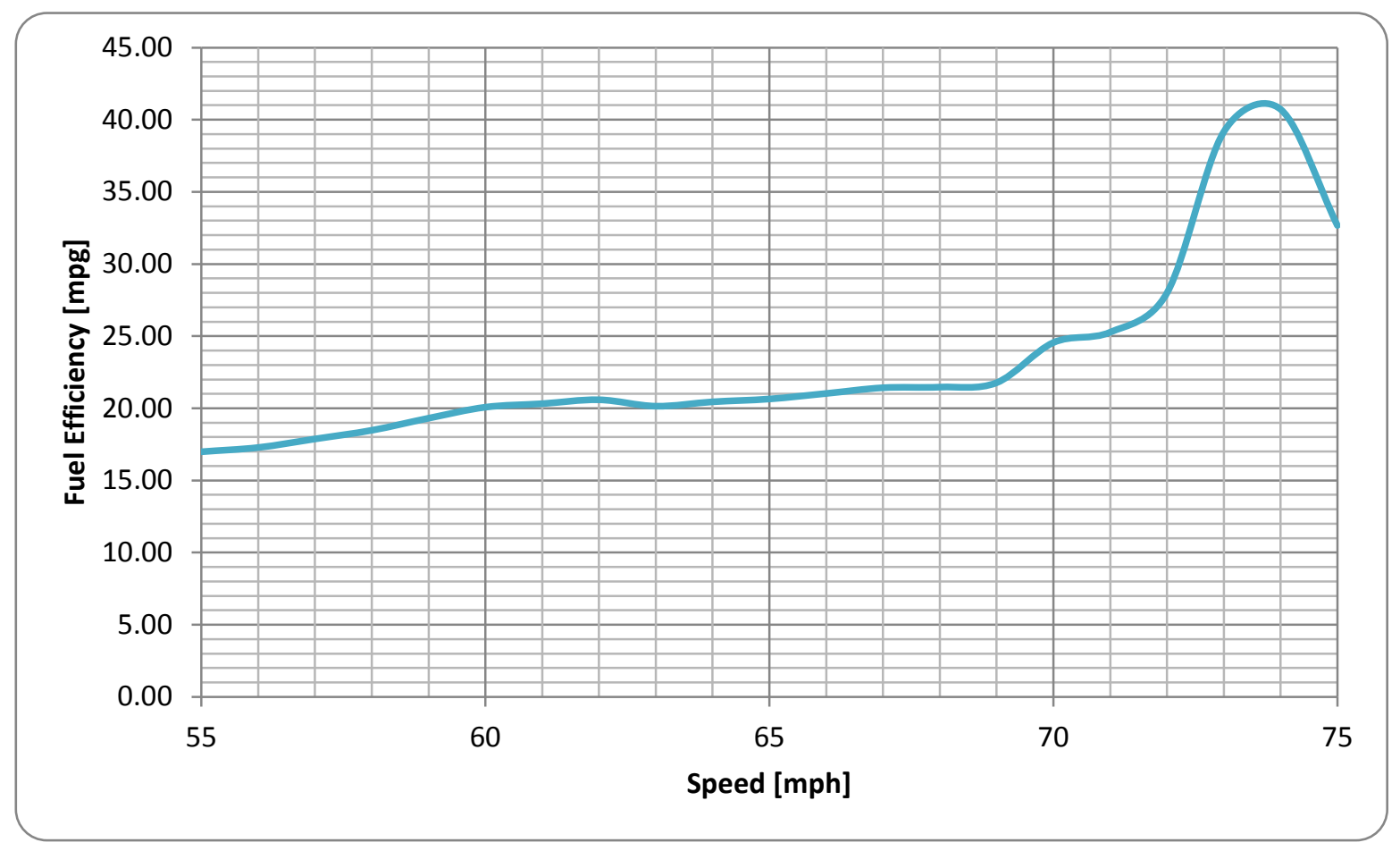

Figure 8. Class-8 Truck Fuel Efficiency by Speed (55 to $75 \mathrm{mph}$ )

Down Slope Terrain (Any Type of Tires, Any Vehicle Weight)

\section{Summary and Conclusions}

Two studies are included in this interim report. In the first one, two vehicle speeds were considered in the computation of Class- 8 truck fuel efficiency, $55 \mathrm{mph}$ and $65 \mathrm{mph}$. The results were disaggregated by the four tractor-trailer tire combinations (i.e., duals-duals, duals-singles, singlesduals, and singles-singles). In all of the cases analyzed, the fuel efficiency obtained at $65 \mathrm{mph}$ was always higher than the fuel efficiency obtained at $55 \mathrm{mph}$. One potential explanation for this finding is that new Class- 8 vehicles are optimized for $65 \mathrm{mph}$ highway speeds, and indeed more miles (four -times) were logged at $65 \mathrm{mph}$ than at $55 \mathrm{mph}$.

In the second part of the study, the fuel efficiency was computed for any speed between 0 and 75 $\mathrm{mph}$, but no consideration was given to the type of tires (i.e., the information was not disaggregated by tractor/trailer tire combination). The fuel efficiencies were computed as the ratio of the distance traveled divided by the total fuel consumed for each one of the categories considered in this study. Analysis of the results indicates that the fuel efficiency peaks at about 59-60 mph and then starts to decrease very slightly until about $70 \mathrm{mph}$.

Future studies that will be performed in this project to analyze the influence of speed on the fuel efficiency of Class-8 trucks will include vehicle weight as well as controlling for terrain profile. 
